\title{
Article
}

\section{Functoriality of the Schmidt construction}

\author{
Juan Climent Vidal ${ }^{1}$ and Enric Cosme Llópez ${ }^{2}$ (D) \\ 1 Universitat de València, Departament de Lògica i Filosofia de la Ciència, Av. Blasco Ibáñez 30-7ª , 46010 \\ València, Spain; Juan.B.Climent@uv.es \\ 2 Universitat de València, Departament de Matemàtiques, Av. Dr. Moliner 50, 46100 Burjassot, Spain; \\ Enric.Cosme@uv.es \\ * Correspondence: Enric.Cosme@uv.es; Tel.: +34-963-544-361
}

\begin{abstract}
After proving, in a purely categorial way, that the inclusion functor $\operatorname{In}_{\operatorname{Alg}(\Sigma)}$ from $\operatorname{Alg}(\Sigma)$, the category of many-sorted $\Sigma$-algebras, to $\operatorname{PAlg}(\Sigma)$, the category of many-sorted partial $\Sigma$-algebras, has a left adjoint $\mathbf{F}_{\Sigma}$, the (absolutely) free completion functor, we recall, in connection with the functor $\mathbf{F}_{\Sigma}$, the generalized recursion theorem of Schmidt, which we will also call the Schmidt construction. Next we define a category $\operatorname{Cmpl}(\Sigma)$, of $\Sigma$-completions, and prove that $\mathbf{F}_{\Sigma}$, labeled with its domain category and the unit of the adjunction of which it is a part, is a weakly initial object in it. Following this we associate to an ordered pair $(\boldsymbol{\alpha}, f)$, where $\alpha=(K, \gamma, \alpha)$ is a morphism of $\Sigma$-completions from $\mathscr{F}=(\mathbf{C}, F, \eta)$ to $\mathscr{G}=(\mathbf{D}, G, \rho)$ and $f$ a homomorphism in $\mathbf{D}$ from the partial $\Sigma$-algebra $\mathbf{A}$ to the partial $\Sigma$-algebra B, a homomorphism $Y_{\alpha}^{\mathscr{E}, 0}(f): \mathbf{S c h}_{\alpha}(f) \longrightarrow$ B. We then prove that there exists an endofunctor, $Y_{\alpha}^{\mathscr{E}, 0}$, of $\mathbf{M o r}_{\mathrm{tw}}(\mathbf{D})$, the twisted morphism category of $\mathbf{D}$, thus showing the naturalness of the previous construction. Afterwards we prove that, for every $\Sigma$-completion $\mathscr{S}=(\mathbf{D}, G, \rho)$, there exists a functor $Y^{\mathscr{G}}$ from the comma category $(\operatorname{Cmpl}(\Sigma) \downarrow \mathscr{G})$ to $\operatorname{End}\left(\operatorname{Mor}_{\mathrm{tw}}(\mathbf{D})\right)$, the category of endofunctors of $\operatorname{Mor}_{\mathrm{tw}}(\mathbf{D})$, such that $Y^{\mathscr{S}, 0}$, the object mapping of $Y^{\mathscr{G}}$, sends a morphism of $\Sigma$-completion in $\operatorname{Cmpl}(\Sigma)$ with codomain $\mathscr{G}$, to the endofunctor $Y_{\alpha}^{\mathscr{E}, 0}$.
\end{abstract}

Keywords: Many-sorted partial algebra, free completion, category of completions, weakly initial object, comma category of objects over a completion, Schmidt construction, Schmidt homomorphism, twisted morphism category, Schmidt endofunctor, functoriality of the Schmidt construction.

Citation: Climent Vidal, J.; Cosme Llópez, E. Functoriality of the Schmidt construction. Preprints 2021, 1, 0. https://doi.org/

Received:

Accepted:

Published:

Publisher's Note: MDPI stays neutral with regard to jurisdictional claims in published maps and institutional affiliations.

\section{Introduction}

Both partiality and heterogeneity are phenomena that occur, since time immemorial, in mathematics and in computability theory. The study of both topics, from an abstract point of view, is carried out, mainly but not exclusively, by means of the many-sorted partial algebras and the homomorphisms between them, i.e., its study takes place in the category $\operatorname{PAlg}(\Sigma)$, of many-sorted partial $\Sigma$-algebras, and subcategories of it. However, such a study is not limited to such categories, but also includes the investigation of the functors from and to such categories, as well as the natural transformations between them. In this regard, there is a key result: The inclusion functor $\operatorname{In}_{\operatorname{Alg}(\Sigma)}$ from $\operatorname{Alg}(\Sigma)$, the category of many-sorted $\Sigma$-algebras, to $\mathbf{P A l g}(\Sigma)$ has a left adjoint $\mathbf{F}_{\Sigma}$, the (absolutely) free completion functor (see [3], [4] and [17]) (and, for every partial $\Sigma$-algebra $\mathbf{A}, \eta^{\mathbf{A}}$, the value of the unit of the adjunction at $\mathbf{A}$, is a dense injective homomorphism from $\mathbf{A}$ into $\mathbf{F}_{\Sigma}(\mathbf{A})$ ). And, in connection with $\mathbf{F}_{\Sigma}$, we have the generalized recursion theorem of Schmidt (see [17]), which we will also call the Schmidt construction, and which states the following:

Let $f$ be a homomorphism from the partial $\Sigma$-algebra $\mathbf{A}$ to the partial $\Sigma$ algebra B. Then there exists an $A$-generated relative subalgebra $\mathbf{S c h}(f)$ of $\mathbf{F}_{\Sigma}(\mathbf{A})$, the free completion of $\mathbf{A}$, and a closed homomorphism $f^{\mathrm{Sch}}: \mathbf{S c h}(f) \longrightarrow \mathbf{B}$ such that $f=f^{\mathrm{Sch}} \circ \operatorname{in}^{\mathbf{A}, \mathbf{S c h}(f)}$, where in $\mathbf{A}^{\mathbf{A}} \mathbf{S c h}(f)$ is the canonical inclusion of $\mathbf{A}$ into $\operatorname{Sch}(f)$. Moreover, $f^{\text {Sch }}$ is the largest homomorphic extension of $f$ to an $A$-generated relative subalgebra of $\mathbf{F}_{\Sigma}(\mathbf{A})$, and it is the only closed one of this kind.

It is worth emphasizing that the Schmidt construction is the basis of a model theoretic approach to the theory of many-sorted partial algebras. Moreover, for every $S$-sorted set $X$, 
every $s \in S$ and every partial $\Sigma$-algebra $\mathbf{A}$, it allows to associate to every term $P$ in $\mathrm{T}_{\Sigma}(X)_{s}$, a partial term operation $P^{\mathbf{A}}$ on $\mathbf{A}$, i.e., a partial mapping from $\operatorname{Hom}(X, A)$, the set of all $S$-sorted mappings from $X$ to $A$, to $A_{s}$; it also allows to state the general homomorphism theorem of Schmidt; and, in addition, given a quasiprimitive set $\mathscr{V}$ of partial $\Sigma$-algebras, i.e., a set of partial $\Sigma$-algebras closed under the operators I, $\mathrm{S}$ and $\mathrm{P}$, it can be used to prove that the inclusion functor $\operatorname{In}_{\mathscr{V}}$ from $\mathscr{V}$, the full subcategory of $\operatorname{PAlg}(\Sigma)$ determined by $\mathscr{V}$, to $\operatorname{PAlg}(\Sigma)$ has a left adjoint $\mathbf{T}_{\mathscr{V}}$.

On the other hand, there exists a functor $(\cdot)^{\infty}$, called the one-point per sort completion, from the category $\operatorname{PAlg}(\Sigma)_{\mathrm{c}}$, of many-sorted partial $\Sigma$-algebras and closed homomorphisms, to the category $\operatorname{Alg}(\Sigma)$ and a natural transformation from the restriction of the functor $\mathbf{F}_{\Sigma}$ to $\operatorname{PAlg}(\Sigma)_{\mathrm{c}}$ to the functor $(\cdot)^{\infty}$. However, and this should be emphasised, for the functor $(\cdot)^{\infty}$, unlike in the case of the functor $\mathbf{F}_{\Sigma}$, there are partial $\Sigma$-algebra $\mathbf{A}$, for which the canonical embedding of $\mathbf{A}$ into $\mathbf{A}^{\infty}$ is not necessarily dense. This deficiency of the functor $(\cdot)^{\infty}$ will lead us to consider a subfunctor $(\cdot)^{\circ}$ of $(\cdot)^{\infty}$ such that, for every partial $\Sigma$-algebra $\mathbf{A}$, the canonical embedding of $\mathbf{A}$ into $\mathbf{A}^{\circ}$ is dense, and which will constitute, together with $\mathbf{F}_{\Sigma}$, another relevant example of the notion of completion of partial $\Sigma$-algebras that we will consider in this paper. Notwithstanding, the functor $(\cdot)^{\infty}$ is used, in particular, in one of the constructive proofs of the recursion theorem for Dedekind-Peano $\Sigma$-algebras with respect to partial $\Sigma$-algebras-which for partial $\Sigma$-algebras is even more important than the recursion theorem for Dedekind-Peano partial $\Sigma$-algebras with respect to $\Sigma$-algebras—and which states the following:

Let $X$ be an $S$-sorted set, A a partial $\Sigma$-algebra and $f$ an $S$-sorted mapping from $X$ to $A$. Then there exists a unique homomorphism

$$
f^{\partial}: \partial(f) \longrightarrow \mathbf{A}
$$

such that

1. $\eta^{X}[X] \subseteq \partial(f)$, where $\eta^{X}$ is the canonical embedding of $X$ into $\mathrm{T}_{\Sigma}(X)$.

2. $\partial(f)$ is an $X\left(\cong \eta^{X}[X]\right)$-generated relative subalgebra of $\mathbf{T}_{\Sigma}(X)$.

3. $f^{\partial} \circ \operatorname{in}^{X, \partial(f)}=f$, where $\operatorname{in}^{X, \partial(f)}$ is the canonical embedding of $X$ into $\partial(f)$.

4. $f^{\partial}$ is a closed homomorphism.

5. $\quad f^{\partial}$ is the largest homomorphic extension of $f$ to an $X$-generated relative subalgebra of $\mathbf{T}_{\Sigma}(X)$ with codomain $\mathbf{A}$.

The observation of the factorizations of the homomorphism $f$ in the generalized recursion theorem of Schmidt and of the many-sorted mapping $f$ in the recursion theorem for Dedekind-Peano $\Sigma$-algebras with respect to partial $\Sigma$-algebras, has led us at first to generalize the Schmidt construction and then to show its functoriality. Such a generalization will be carried out through the notions of $\Sigma$-completion and of morphism between $\Sigma$-completions and it will consist in associating to a pair formed by a morphism of $\Sigma$-completions and a homomorphism between partial $\Sigma$-algebras, a so-called Schmidt homomorphism, concretely, we will state and prove the following proposition:

Let $\alpha=(K, \gamma, \alpha)$ be a morphism of $\Sigma$-completions from $\mathscr{F}=(\mathbf{C}, F, \eta)$ to $\mathscr{G}=(\mathbf{D}, G, \rho)$ and $f: \mathbf{A} \longrightarrow \mathbf{B}$ a homomorphism of $\mathbf{D}$. Then there exists an $\eta^{K(\mathbf{A})}\left[\gamma^{\mathbf{A}}[A]\right]$-generated relative subalgebra $\mathbf{S c h}_{\alpha}(f)$ of $F(K(\mathbf{A}))$, the $\Sigma$-completion of $K(\mathbf{A})$ associated to $\mathscr{F}$, and a homomorphism $Y_{\alpha}^{\mathscr{G}, 0}(f)$ of $\mathbf{D}$ from $\mathbf{S c h}_{\alpha}(f)$ to $\mathbf{B}$ such that $f=Y_{\alpha}^{\mathscr{G}, 0}(f) \circ\left(\eta^{K(\mathbf{A})} \circ \gamma^{\mathbf{A}}\right)$, where, with the customary abuse of notation, the same symbol is used for the homomorphism $\eta^{K(\mathbf{A})} \circ \gamma^{\mathbf{A}}$ from $\mathbf{A}$ to $F(K(\mathbf{A}))$ and its corestriction to $\mathbf{S c h}_{\alpha}(f)$. Moreover, $\mathrm{Y}_{\alpha}^{\mathscr{G}, 0}(f)$ is the largest homomorphic extension of $f$ to an $\eta^{K(\mathbf{A})}\left[\gamma^{\mathbf{A}}[A]\right]$-generated relative subalgebra of $F(\mathbf{A})$.

The plan of this paper is, briefly, as follows. 
In Sections 2 to 5 we fix notation and terminology and review those concepts and results about many-sorted sets, many-sorted algebras, many-sorted partial algebras and partial Dedekind-Peano algebras, which will be used in this paper and which will make it as self-contained as possible (this material is quite standard, so the expert reader may skip most of it).

In Section 6, taking into account a theorem about adjoint functors, we again obtain, as a corollary of it, the theorem that states that the inclusion functor $\operatorname{In}_{\operatorname{Alg}(\Sigma)}$ from $\operatorname{Alg}(\Sigma)$ to $\operatorname{PAlg}(\Sigma)$ has a left adjoint $\mathbf{F}_{\Sigma}$, the (absolutely) free completion functor. At the end of this section, and related to the functor $\mathbf{F}_{\Sigma}$ from $\operatorname{PA} \lg (\Sigma)$ to $\operatorname{Alg}(\Sigma)$, we state the generalized recursion theorem of Schmidt (see [17]), which we will also call the Schmidt construction. We remark that the rest of this article will focus on such a nice and fundamental mathematical construction in order to show its functoriality.

Next, in Section 7, and assuming that the category $\operatorname{PAlg}(\Sigma)$ is equipped with a factorization system $(\mathbf{E}, \mathbf{M})$, we define a category $\operatorname{Cmpl}(\Sigma)$, of $\Sigma$-completions, whose objects are ordered triples $\mathscr{F}=(\mathbf{C}, F, \eta)$ that satisfy the following conditions:

1. $\mathbf{C}$ is a wide subcategory of $\operatorname{PAlg}(\Sigma)$, i.e., a subcategory of $\mathbf{P A l g}(\Sigma)$ such that $\mathrm{Ob}(\mathbf{C})=$ $\mathrm{Ob}(\operatorname{PAlg}(\Sigma))$, satisfying that

1.1 for every homomorphism $f$ of $\mathbf{C}$ if $f=h \circ g$, for some $(g, h) \in \mathbf{E} \times \mathbf{M}$, then $h$ is a homomorphism of $\mathbf{C}$.

2. $\quad F$ is a functor from $\mathrm{C}$ to $\operatorname{Alg}(\Sigma)$.

3. $\quad \eta$ is a natural transformation from $\operatorname{In}_{C}$ to $\operatorname{In}_{\mathrm{Alg}(\Sigma)} \circ F$, i.e., for every homomorphism $f: \mathbf{A} \longrightarrow \mathbf{B}$ between partial $\Sigma$-algebras of $\mathbf{C}$, we have that

$$
F(f) \circ \eta^{\mathbf{A}}=\eta^{\mathbf{B}} \circ f
$$

satisfying that

3.1 for every partial $\Sigma$-algebra $\mathbf{A}, \eta^{\mathbf{A}}: \mathbf{A} \longrightarrow F(\mathbf{A})$ is a dense injective homomorphism.

Moreover, we prove that $\mathscr{F}_{\Sigma}=\left(\operatorname{PAlg}(\Sigma), \mathbf{F}_{\Sigma}, \eta\right)$, the $\Sigma$-completion associated to the free completion functor $\mathbf{F}_{\Sigma}$ and the unit $\eta$ of the adjunction $\mathbf{F}_{\Sigma} \dashv \operatorname{In} \mathbf{A l g}(\Sigma)$, is a weakly initial object of $\operatorname{Cmpl}(\Sigma)$.

Following this, in Section 8, we begin to realize our project of showing the functoriality of the Schmidt construction. To do it, we begin by associating to an ordered pair $(\alpha, f)$, where $\boldsymbol{\alpha}=(K, \gamma, \alpha)$ is a morphism of $\Sigma$-completions from $\mathscr{F}=(\mathbf{C}, F, \eta)$ to $\mathscr{G}=(\mathbf{D}, G, \rho)$ and $f$ a homomorphism of $\mathbf{D}$ from the partial $\Sigma$-algebra $\mathbf{A}$ to the partial $\Sigma$-algebra $\mathbf{B}$, a homomorphism $\mathrm{Y}_{\alpha}^{\mathscr{G}, 0}(f): \mathbf{S c h}_{\alpha}(f) \longrightarrow \mathbf{B}$. This construction, actually, generalizes the Schmidt construction. We then prove that there exists an endofunctor, denoted $Y_{\alpha}^{\mathscr{G}, 0}$, called the Schmidt endofunctor relative to $\mathscr{G}$ and $\boldsymbol{\alpha}$, of $\mathbf{M o r}_{\mathrm{tw}}(\mathbf{D})$, the twisted morphism category of $\mathbf{D}$, thus showing the naturalness of the Schmidt construction.

Afterwards, in Section 9, we prove that, for every $\Sigma$-completion $\mathscr{G}$, there exists a functor $\mathrm{Y}^{\mathscr{G}}$ from the comma category $(\operatorname{Cmpl}(\Sigma) \downarrow \mathscr{G})$ to $\operatorname{End}\left(\operatorname{Mor}_{\mathrm{tw}}(\mathbf{D})\right)$, the category of endofunctors of $\mathbf{M o r}_{\mathrm{tw}}(\mathbf{D})$, such that $\mathrm{Y}^{\mathscr{G}, 0}$, the object mapping of $\mathrm{Y}^{\mathscr{G}}$, sends an object $\alpha: \mathscr{F} \longrightarrow \mathscr{G}$ of $(\operatorname{Cmpl}(\Sigma) \downarrow \mathscr{G})$, i.e., a morphism of $\Sigma$-completion of $\operatorname{Cmpl}(\Sigma)$ with codomain $\mathscr{G}$, to the endofunctor $\mathrm{Y}_{\alpha}^{\mathscr{G}, 0}$ of $\operatorname{End}\left(\operatorname{Mor}_{\mathrm{tw}}(\mathbf{D})\right)$.

Although there are excellent monographs and survey articles on many-sorted partial algebras, e.g., [5] and [6], unfortunately it does not seem that, generally speaking, researchers are familiar enough with such a mathematical field. So it has seemed to us appropriate, for completeness and easy reference, to recall those fundamental notions and constructions in that field of which we make use.

Our underlying set theory is ZFSk, Zermelo-Fraenkel-Skolem set theory (also known as ZFC, i.e., Zermelo-Fraenkel set theory with the axiom of choice) plus the existence of a Grothendieck universe $\mathcal{U}$, fixed once and for all (see [14], pp. 21-24). We recall that the elements of $\mathcal{U}$ are called $\mathcal{U}$-small sets and the subsets of $\mathcal{U}$ are called $\mathcal{U}$-large sets or classes. 
Moreover, from now on Set stands for the category of sets, i.e., the category whose set of objects is $\mathcal{U}$ and whose set of morphisms is the set of all mappings between $\mathcal{U}$-small sets.

In all that follows we use standard concepts and constructions from category theory, see e.g., [12] and [14], universal algebra, see e.g., [1], [5] [6], [7], [8], [10], [11], [13], [15], [16], [17] and [18], and set theory, see e.g., [2]. Nevertheless, regarding set theory, we have adopted the following conventions. An ordinal $\alpha$ is a transitive set that is well-ordered by $\in$, thus $\alpha=\{\beta \mid \beta \in \alpha\}$. The first transfinite ordinal $\omega_{0}$ will be denoted by $\mathbb{N}$, which is the set of all natural numbers, and, from what we have just said about the ordinals, for every $n \in \mathbb{N}, n=\{0, \ldots, n-1\}$. If $\Phi$ and $\Psi$ are (binary) relations in a set $A$, then we will say that $\Psi$ is a refinement of $\Phi$ if $\Psi \subseteq \Phi$. We will denote by $\operatorname{Pfnc}(A, B)$ the set of all partial functions from $A$ to $B$, and by $\operatorname{Fnc}(A, B)$ the set of all functions from $A$ to $B$. We recall that a partial function from $A$ to $B$ is a subset $F$ of $A \times B$ such that, for every $x \in A$, there is at most one $y \in B$ such that $(x, y) \in F$, and that a function from $A$ to $B$ is a subset $F$ of $A \times B$ such that, for every $x \in A$, there exists a unique $y \in B$ such that $(x, y) \in F$. A partial function from $A$ to $B$ is usually denoted by $\left(F_{x}\right)_{x \in \operatorname{Dom}(F) \text {, where }}$ $\operatorname{Dom}(F)$, the domain (of definition) of $F$, is $\{x \in A \mid \exists y \in B((x, y) \in F)\}$, and a function $F$ from $A$ to $B$ is generally denoted by $\left(F_{x}\right)_{x \in A}$. We will denote by $\operatorname{Hom}_{\mathrm{p}}(A, B)$ the set of all partial mappings from $A$ to $B$, and by $\operatorname{Hom}(A, B)$ (and, sometimes, also by $B^{A}$ ) the set of all mappings from $A$ to $B$. We recall that a partial mapping from $A$ to $B$ is an ordered triple $f=(A, F, B)$, denoted by $f: A-B$, in which $F$ is a partial function from $A$ to $B$, and that a mapping from $A$ to $B$ is an ordered triple $f=(A, F, B)$, denoted by $f: A \longrightarrow B$, in which $F$ is a function from $A$ to $B$. Therefore $\operatorname{Hom}_{\mathrm{p}}(A, B)=\{A\} \times \operatorname{Pfnc}(A, B) \times\{B\}$ and $\operatorname{Hom}(A, B)=\{A\} \times \operatorname{Fnc}(A, B) \times\{B\}$. For a partial mapping $f: A \longrightarrow B$ we will denote by $\Gamma_{f}$ the underliyng partial function of $f$ and by $\operatorname{Dom}(f)$ precisely $\operatorname{Dom}\left(\Gamma_{f}\right)$. For two sets $A$ and $B$ we will denote by $0_{A, B}$ the partial mapping from $A$ to $B$ such that $\operatorname{Dom}\left(0_{A, B}\right)=\varnothing\left(\equiv \Gamma_{0_{A, B}}=\varnothing\right)$. Thus $0_{A, B}$ is the composition of the unique partial mapping from $A$ to $\varnothing$ and the unique (partial) mapping from $\varnothing$ to $B$. Let us note that, for a partial mapping $f: A \longrightarrow B, \mathrm{~d}_{0}(f)$, the categorial domain (or source) of $f$, which is $A$, contains $\operatorname{Dom}(f)$, while, for a mapping $f: A \longrightarrow B, \mathrm{~d}_{0}(f)=\operatorname{Dom}(f)$. We will denote by $\operatorname{Sub}(A)$ the set of all sets $X$ such that $X \subseteq A$ and if $X \in \operatorname{Sub}(A)$, then we will denote by $\complement_{A} X$ or $A-X$ the complement of $X$ in $A$. Moreover, if $f$ is a mapping from $A$ to $B$, then the mapping $f[\cdot]$ from $\operatorname{Sub}(A)$ to $\operatorname{Sub}(B)$, of $f$-direct image formation, sends $X$ in $\operatorname{Sub}(A)$ to $f[X]=\{y \in B \mid \exists x \in X(y=f(x))\}$ in $\operatorname{Sub}(B)$, and the mapping $f^{-1}[\cdot]$ from $\operatorname{Sub}(B)$ to $\operatorname{Sub}(A)$, of $f$-inverse image formation, sends $Y$ in $\operatorname{Sub}(B)$ to $f^{-1}[Y]=\{x \in A \mid f(x) \in Y\}$ in $\operatorname{Sub}(A)$. In the sequel, for a mapping $f$ from $A$ to $B$ and a subset $X$ of $A$, we will write $\operatorname{Ker}(f)$ for the kernel of $f, \operatorname{Im}(f)$ to mean $f[A]$ and the restriction of $f$ to $X$ will be denoted by $f \uparrow_{X}$. Let us note that for partial mappings $f: A-B$ and $g: B-C$, the domain of the composite $g \circ f$ is $\operatorname{Dom}(g \circ f)=f^{-1}[\operatorname{Dom}(g) \cap f[\operatorname{Dom}(f)]]$ and the image is $\operatorname{Im}(g \circ f)=g[\operatorname{Dom}(g) \cap f[\operatorname{Dom}(f)]]$.

Finally, with regard to category theory, we have adopted the following conventions. If $\mathbf{C}$ is a category, then $\mathrm{Ob}(\mathbf{C})$ denotes the set of objects of $\mathbf{C}$, and $\operatorname{Mor}(\mathbf{C})$ its set of morphisms. Moreover, the operations of vertical and horizontal composition of natural transformations will be denoted by $\circ$ and $*$, respectively.

\section{Many-sorted sets}

In this section we collect the basic facts, mostly without proofs, about many-sorted sets that we will need afterwards.

Assumption 1. From now on $S$ stands for a set of sorts in $\mathcal{U}$, fixed once and for all.

Definition 1. An $S$-sorted set is a mapping $A=\left(A_{S}\right)_{s \in S}$ from $S$ to $\mathscr{U}$. 
Definition 2. If $A$ and $X$ are $S$-sorted sets, then we will say that $X$ is a subset of $A$, denoted by $X \subseteq A$, if, for every $s \in S, X_{S} \subseteq A_{s}$. We will denote by $\operatorname{Sub}(A)$ the set of all $S$-sorted sets $X$ such that $X \subseteq A$.

Definition 3. Let $A$ and $B$ be $S$-sorted sets. The cartesian product of $A$ and $B$, denoted by $A \times B$, is the $S$-sorted set $\left(A_{s} \times B_{S}\right)_{s \in S}$.

Let $\Phi$ be an $S$-sorted set. We will say that $\Phi$ is an $S$-sorted relation from $A$ to $B$ if $\Phi \subseteq A \times B$. Thus, for every $s \in S, \Phi_{S} \subseteq A_{S} \times B_{S}$. We denote by $\operatorname{Rel}(A, B)$ the set of all $S$-sorted relations from $A$ to $B$. If $A=B$, then we write $\operatorname{Rel}(A)$ instead of $\operatorname{Rel}(A, A)$ and call its elements $S$-sorted relations on $A$.

The diagonal of $A$, denoted by $\Delta_{A}$, is the $S$-sorted relation on $A$ defined, for every $s \in S$, as $\Delta_{A_{S}}$, and the codiagonal of $A$, denoted by $\nabla_{A}$, is the $S$-sorted relation on $A$ defined, for every $s \in S$, as $\nabla_{A_{s}}=A_{s} \times A_{s}$.

Let $\Phi$ be an $S$-sorted relation from $A$ to $B$ and $\Psi$ an $S$-sorted relation from $B$ to $C$, then the composition of $\Phi$ and $\Psi$, denoted by $\Psi \circ \Phi$, is the $S$-sorted relation from $A$ to $C$ defined, for every $s \in S$, as

$$
\Psi_{S} \circ \Phi_{S}=\left\{(x, z) \in A_{S} \times C_{s} \mid \exists y \in B_{S}\left((x, y) \in \Phi_{S} \&(y, z) \in \Psi_{S}\right)\right\} .
$$

This composition is associative and the diagonal relation $\Delta_{A}$ is a neutral element for it.

Let $\Phi$ an $S$-sorted relation from $A$ to $B$. Then the inverse of $\Phi$, denoted by $\Phi^{-1}$, is the $S$-sorted relation from $B$ to $A$ defined, for every $s \in S$, as

$$
\Phi_{s}^{-1}=\left\{(y, x) \in B_{s} \times A_{s} \mid(x, y) \in \Phi_{s}\right\} .
$$

Let $\Phi$ be an $S$-sorted relation from $A$ to $B, X$ an S-sorted subset of $A$ and $Y$ an $S$-sorted subset of $B$, then the direct image of $X$ under $\Phi$, denoted by $\Phi[X]$, is the $S$-sorted subset of $B$ defined, for every $s \in S$, as

$$
\Phi[X]_{S}=\left\{b \in B_{S} \mid \exists x \in X_{S}\left((x, b) \in \Phi_{S}\right)\right\},
$$

therefore $\Phi[X]=\left(\Phi_{S}\left[X_{S}\right]\right)_{s \in S}$, and the inverse image of $Y$ under $\Phi$, denoted by $\Phi^{-1}[Y]$, is the $S$-sorted subset of $A$ defined, for every $s \in S$, as

$$
\Phi^{-1}[Y]_{S}=\left\{a \in A_{s} \mid \exists y \in X_{S}\left((a, y) \in \Phi_{s}\right)\right\},
$$

therefore $\Phi^{-1}[Y]=\left(\Phi_{S}^{-1}\left[Y_{S}\right]\right)_{s \in S}$.

$A n S$-sorted function from $A$ to $B$ is a functional $S$-sorted relation $F$ from $A$ to $B$, i.e., an $S$-sorted relation $F$ from $A$ to $B$ such that, for every $s \in S, F_{S}$ is a function from $A_{S}$ to $B_{S}$. We denote by $\operatorname{Fnc}(A, B)$ the set of all $S$-sorted functions from $A$ to $B$. The composition of $S$-sorted functions, which is a particular case of the composition of $S$-sorted relations, is an S-sorted function.

An $S$-sorted mapping from $A$ to $B$ is a triple $f=(A, F, B)$ where $F$ is an S-sorted function from $A$ to $B$. We denote by $\operatorname{Hom}(A, B)$ or by $B_{A}$ the set of all $S$-sorted mappings from $A$ to $B$. We consider the expressions $f \in \operatorname{Hom}(A, B), f \in B_{A}$ and $f: A \longrightarrow B$ as synonymous. Moreover, given $f: A \longrightarrow B$ and $g: B \longrightarrow C, g \circ f=(A, G \circ F, C)$, the composition of $f$ and $g$, is an $S$-sorted mapping from $A$ to $C$, and $\mathrm{id}_{A}=\left(A, \Delta_{A}, A\right)$, is an S-sorted endomapping of $A$, the identity $S$-sorted mapping at $A$. We denote by $\mathbf{S e t}^{S}$ the category of $S$-sorted sets and $S$-sorted mappings.

Let $f: A \longrightarrow B$ be an S-sorted mapping. Then the mapping

$$
f[\cdot]: \operatorname{Sub}(A) \longrightarrow \operatorname{Sub}(B),
$$

of $f$-direct image formation, sends $X \in \operatorname{Sub}(A)$ to $f[X]=\left(f_{s}\left[X_{s}\right]\right)_{s \in S} \in \operatorname{Sub}(B)$, and the mapping

$$
f^{-1}[\cdot]: \operatorname{Sub}(B) \longrightarrow \operatorname{Sub}(A)
$$

of $f$-inverse image formation, sends $Y \in \operatorname{Sub}(B)$ to $f^{-1}[Y]=\left(f_{S}^{-1}\left[Y_{S}\right]\right)_{s \in S} \in \operatorname{Sub}(A)$. 


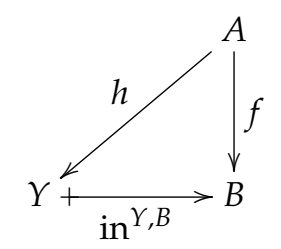

Figure 1. The corestriction of a many-sorted mapping.

The image of $f$, denoted by $\operatorname{Im}(f)$, is $f[A]$, i.e., $\left(f_{S}\left[A_{s}\right]\right)_{s \in S}$. Moreover, if $X \subseteq A$, then the restriction of $f$ to $X$, denoted by $\left.f\right|_{X},\left.f\right|_{X}$ or $\operatorname{res}_{X}(f)$, is $f \circ$ in $^{X, A}$, where in ${ }^{X, A}=\left(\text { in }^{X_{s}, A_{s}}\right)_{s \in S}$ is the canonical embedding of $X$ into $A$.

Remark 1. To give an $S$-sorted mapping from $A$ to $B$, as in the just stated definition, is equivalent to give an $S$-indexed family $f=\left(f_{s}\right)_{s \in S}$, where, for every $s$ in $S, f_{S}$ is a mapping from $A_{S}$ to $B_{S}$. Thus, an $S$-sorted mapping from $A$ to $B$ is, essentially, an element of $\prod_{s \in S} \operatorname{Hom}\left(A_{s}, B_{s}\right)$.

Definition 4. Let $I$ be $a$ set in $\mathcal{U}$ and $\left(A^{i}\right)_{i \in I}$ an I-indexed family of S-sorted sets. Then the product of $\left(A^{i}\right)_{i \in I}$, denoted by $\prod_{i \in I} A^{i}$, is the $S$-sorted set defined, for every $S \in S$, as $\left(\prod_{i \in I} A^{i}\right)_{s}=\prod_{i \in I} A_{s}^{i}$, where

$$
\prod_{i \in I} A_{s}^{i}=\left\{\left(a_{i}\right)_{i \in I} \in \operatorname{Fnc}\left(I, \bigcup_{i \in I} A_{s}^{i}\right) \mid \forall i \in I\left(a_{i} \in A_{s}^{i}\right)\right\} .
$$

For every $i \in I$, the $\mathrm{i}$-th canonical projection, $\mathrm{pr}^{i}=\left(\mathrm{pr}_{s}^{i}\right)_{s \in S}$, is the S-sorted mapping from $\prod_{i \in I} A^{i}$ to $A^{i}$ that, for every $s \in S$, sends $\left(a_{i}\right)_{i \in I}$ in $\prod_{i \in I} A_{s}^{i}$ to $a_{i}$ in $A_{s}^{i}$. The ordered pair $\left(\prod_{i \in I} A^{i},\left(\mathrm{pr}^{i}\right)_{i \in I}\right)$ has the following universal property: For every $S$-sorted set $B$ and every Iindexed family of $S$-sorted mappings $\left(f^{i}\right)_{i \in I}$, where, for every $i \in I, f^{i}$ is an $S$-sorted mapping from $B$ to $A^{i}$, there exists a unique $S$-sorted mapping $\left\langle f^{i}\right\rangle_{i \in I}$ from $B$ to $\prod_{i \in I} A^{i}$ such that, for every $i \in I, \operatorname{pr}^{i} \circ\left\langle f^{i}\right\rangle_{i \in I}=f^{i}$.

The coproduct of $\left(A^{i}\right)_{i \in I}$, denoted by $\coprod_{i \in I} A^{i}$, is the $S$-sorted set defined, for every $s \in S$, as $\left(\coprod_{i \in I} A^{i}\right)_{s}=\coprod_{i \in I} A_{s}^{i}$, where

$$
\coprod_{i \in I} A_{S}^{i}=\bigcup_{i \in I}\left(A_{S}^{i} \times\{i\}\right) .
$$

For every $i \in I$, the $\mathrm{i}$-th canonical injection, in ${ }^{i}$, is the $S$-sorted mapping from $A^{i}$ to $\coprod_{i \in I} A^{i}$ that, for every $s \in S$, sends a in $A_{s}^{i}$ to $(a, i)$ in $\bigsqcup_{i \in I} A_{s}^{i}$. The ordered pair $\left(\coprod_{i \in I} A^{i},\left(\mathrm{in}^{i}\right)_{i \in I}\right)$ has the following universal property: For every $S$-sorted set $B$ and every I-indexed family of $S$-sorted mappings $\left(f^{i}\right)_{i \in I}$, where, for every $i \in I, f^{i}$ is an $S$-sorted mapping from $A^{i}$ to $B$, there exists a unique $S$-sorted mapping $\left[f^{i}\right]_{i \in I}$ from $\bigsqcup_{i \in I} A^{i}$ to $B$ such that, for every $i \in I,\left[f^{i}\right]_{i \in I} \circ$ in $^{i}=f^{i}$.

The remaining set-theoretic operations on $S$-sorted sets: $\amalg$ (binary coproduct), $\cup$ (union), $\cup$ (binary union), $\cap$ (intersection), $\cap$ (binary intersection), - (difference) and $\complement_{A}$ (complement of an $S$-sorted set in a fixed $S$-sorted $A$ ), are defined in a similar way, i.e., componentwise.

Definition 5. We will denote by $1^{S}$ the (standard) final $S$-sorted set of $\mathbf{S e t}^{S}$, which is $1^{S}=(1)_{s \in S \text {, }}$ and by $\varnothing^{S}$ the initial $S$-sorted set, which is $\varnothing^{S}=(\varnothing)_{s \in S}$. We shall abbreviate $1^{S}$ to 1 and $\varnothing^{S}$ to $\varnothing$ when this is unlikely to cause confusion.

Proposition 1. Let $B$ be an S-sorted set, $Y$ a subset of $B$ and $f$ an $S$-sorted mapping from $A$ to $B$. Then the following statements are equivalent:

1. $\operatorname{Im}(f) \subseteq Y$.

2. There exists an $S$-sorted mapping $h$ from $A$ to $Y$ such that the diagram in Figure 1 commutes. If one of the above equivalent statements holds, then we will call $h$, which is univocally determined, the corestriction of $f$ to $Y$ and we denote it by $\left.f\right|^{Y}$ or $\operatorname{cores}_{Y}(f)$. 


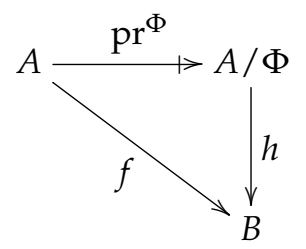

Figure 2. The coastriction of a many-sorted mapping.

Proposition 2. Let PSet $^{S}$ be the category whose objects are the $S$-sorted set pairs, i.e., the ordered pairs pairs $(A, X)$ where $A$ is an $S$-sorted set and $X \subseteq A$, and in which the set of morphisms from $(A, X)$ to $(B, Y)$ is the set of all S-sorted mappings from $A$ to $B$ such that $f[X] \subseteq Y$. Let $G$ be the functor from $\mathbf{S e t}^{S}$ to $\mathbf{P S e t}^{S}$ whose object mapping sends $A$ to $(A, A)$ and whose morphism mapping sends $f: A \longrightarrow B$ to $f:(A, A) \longrightarrow(B, B)$. Then, for every $S$-sorted pair $(B, Y)$, there exists a universal mapping from $G$ to $(B, Y)$, which is precisely the ordered pair $\left(Y\right.$, in $\left.^{Y, B}\right)$ with $\operatorname{in}_{Y, B}:(Y, Y) \longrightarrow(B, Y)$ the morphism of PSet $^{S}$ associated to in ${ }^{Y, B}: Y \longrightarrow B$.

Definition 6. Let $f$ be a $\mathbf{P S e t}^{S}$-morphism from $(A, X)$ to $(B, Y)$. Then we denote by $\left.f\right|_{X^{\prime}} ^{Y}$ bires $_{X, Y}(f)$, or, if no confusion can arise, $\widehat{f}$ the S-sorted mapping cores ${ }_{Y}\left(\operatorname{res}_{X}(f)\right.$ ) (which is identical to $\left.\operatorname{res}_{X}\left(\operatorname{cores}_{Y}(f)\right)\right)$. We will call this S-sorted mapping the birestriction of $f$ to $X$ and $Y$.

Definition 7. Let $A$ be an $S$-sorted set. Then the cardinal of $A$, denoted by $\operatorname{card}(A)$, is card $(\amalg A)$, i.e., the cardinal of the set $\amalg A=\bigcup_{s \in S}\left(A_{S} \times\{s\}\right)$. An S-sorted set $A$ is finite if $\operatorname{card}(A)<\aleph_{0}$. We will say that an $S$-sorted set $X$ is a finite subset of $A$ if $X$ is finite and $X \subseteq A$. We will denote by $\operatorname{Sub}_{\mathrm{f}}(A)$ the set of all $S$-sorted sets $X$ in $\operatorname{Sub}(A)$ which are finite.

Definition 8. Let $A$ be an $S$-sorted set. Then the support of $A$, denoted by $\operatorname{supp}_{S}(A)$, is the set $\left\{s \in S \mid A_{s} \neq \varnothing\right\}$.

Remark 2. An S-sorted set $A$ is finite if and only if $\operatorname{supp}_{S}(A)$ is finite and, for every $s \in$ $\operatorname{supp}_{S}(A), A_{S}$ is finite.

We next define the notion of equivalence relation on a many-sorted set and state the universal property of the corresponding quotient many-sorted set.

Definition 9. An $S$-sorted equivalence relation on (or, to abbreviate, an $S$-sorted equivalence on) an $S$-sorted set $A$ is an $S$-sorted relation $\Phi$ on $A$ such that, for every $s \in S, \Phi_{s}$ is an equivalence relation on $A_{s}$. We will denote by $\operatorname{Eqv}(A)$ the set of all $S$-sorted equivalences on $A$ (which is an algebraic closure system on $A \times A)$, by $\operatorname{Eqv}(A)$ the algebraic lattice $(\operatorname{Eqv}(A), \subseteq)$, by $\nabla_{A}$ the greatest element of $\operatorname{Eqv}(A)$ and by $\Delta_{A}$ the least element of $\operatorname{Eqv}(A)$. As for ordinary sets, $\operatorname{Eqv}(A)$ is also an algebraic lattice, and we denote by $\mathrm{Eg}_{A}$ the canonically associated algebraic closure operator. For $A$, we have that $\operatorname{Eg}_{A}(\Phi)=\left(\operatorname{Eg}_{A_{S}}\left(\Phi_{S}\right)\right)_{s \in S}$.

For an $S$-sorted equivalence relation $\Phi$ on $A$, the $S$-sorted quotient set of $A$ by $\Phi$, denoted by $A / \Phi$, is $\left(A_{S} / \Phi_{S}\right)_{s \in S}=\left(\left\{[x]_{\Phi_{s}} \mid x \in A_{S}\right\}\right)_{s \in S}\left(\subseteq\left(\operatorname{Sub}\left(A_{S}\right)\right)_{s \in S}\right)$, where, for every $s \in S$ and every $x \in A_{S},[x]_{\Phi_{s}}$, the equivalence class of $x$ with respect to $\Phi_{s}$ (or, the $\Phi$-equivalence class of $x)$ is $\left\{y \in A_{s} \mid(x, y) \in \Phi_{s}\right\}$, and $\operatorname{pr}^{\Phi}: A \longrightarrow A / \Phi$, the canonical projection from $A$ to $A / \Phi$, is the $S$-sorted mapping $\left(\mathrm{pr}_{s}^{\Phi}\right)_{s \in S}$, where, for every $s \in S, \mathrm{pr}_{s}^{\Phi}$ is the canonical projection from $A_{S}$ to $A_{S} / \Phi_{S}$ (which sends $x$ in $A_{S}$ to $\operatorname{pr}_{S}^{\Phi}(x)=[x]_{\Phi_{s}}$, the $\Phi_{s}$-equivalence class of $x$, in $\left.A_{S} / \Phi_{S}\right)$. Moreover, if $\Psi$ is an $S$-sorted equivalence on an $S$-sorted set $B$ and $f$ an $S$-sorted mapping from $A$ to $B$, then the astriction of $f$ to $B / \Psi$, denoted by $\operatorname{ast}_{B / \Psi}(f)$, is $\operatorname{pr}^{\Psi} \circ f$, where $\operatorname{pr}^{\Phi}$ is the canonical projection of $B$ onto $B / \Psi$. 
Proposition 3. Let $A$ be an $S$-sorted set, $\Phi$ an S-sorted equivalence on $A$ and $f: A \longrightarrow B$ an $S$-sorted mapping. Then the following statements are equivalent:

1. $\Phi \subseteq \operatorname{Ker}(f)$.

2. There exists an S-sorted mapping $h$ from $A / \Phi$ to $B$ such that the diagram in Figure 2 commutes.

If one of the above equivalent statements holds, then we will call $h$, which is univocally determined, the coastriction of $f$ to $A / \Phi$ and we denote it by coast $_{A / \Phi}(f)$.

Proposition 4. Let $\mathrm{ClfdSet}^{S}$ be the category whose objects are the classified $S$-sorted sets, i.e, the ordered pairs $(A, \Phi)$ where $A$ is an $S$-sorted set and $\Phi$ an $S$-sorted equivalence relation on $A$, and in which the set of morphisms from $(A, \Phi)$ to $(B, \Psi)$ is the set of all S-sorted mappings $f$ from $A$ to $B$ such that, for every $s \in S$ and every $(x, y) \in A_{s}^{2}$, if $(x, y) \in \Phi_{s}$, then $\left(f_{s}(x), f_{s}(y)\right) \in \Psi_{s}$. Let $G$ be the functor from $\mathbf{S e t}^{S}$ to $\mathbf{C l f d S e t}^{S}$ whose object mapping sends $A$ to $\left(A, \Delta_{A}\right)$ and whose morphism mapping sends $f: A \longrightarrow B$ to $f:\left(A, \Delta_{A}\right) \longrightarrow\left(B, \Delta_{B}\right)$. Then, for every classified $S$ sorted set $(A, \Phi)$, there exists a universal mapping from $(A, \Phi)$ to $G$, which is precisely the ordered $\operatorname{pair}\left(A / \Phi, \mathrm{pr}^{\Phi}\right)$ with $\mathrm{pr}^{\Phi}:(A, \Phi) \longrightarrow\left(A / \Phi, \Delta_{A} / \Phi\right)$.

Definition 10. Let $f$ be a ClfdSet $^{S}$-morphism from $(A, \Phi)$ to $(B, \Psi)$. Then we denote by biast $_{A / \Phi, B / \Psi}(f)$ or $f^{\Phi, \Psi}$ the S-sorted mapping $\operatorname{ast}_{B / \Psi}\left(\operatorname{coast}_{A / \Phi}(f)\right)$ (which is identical to coast $_{A / \Phi}\left(\operatorname{ast}_{B / \Psi}(f)\right)$ ). We will call this S-sorted mapping the biastriction of $f$ to $A / \Phi$ and $B / \Psi$.

We define next the concept of kernel of an $S$-sorted mapping, and state the quadrangular and triangular factorizations of an $S$-sorted mapping.

Definition 11. let $f: A \longrightarrow B$ be an S-sorted mapping. Then the kernel of $f$, denoted by $\operatorname{Ker}(f)$, is the $S$-equivalence on $A$ defined as:

$$
\operatorname{Ker}(f)=\left(\operatorname{Ker}\left(f_{s}\right)\right)_{s \in S} .
$$

Proposition 5. Let $f: A \longrightarrow B$ be an S-sorted mapping. Then we have the following quadrangular and triangular factorizations of $f$ :

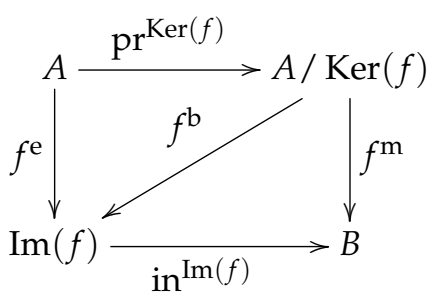

where the involved $S$-sorted mappings are defined coordinatewise, i.e., for every $s \in S,\left(\operatorname{pr}^{\operatorname{Ker}(f)}\right)_{s}$ is $\mathrm{pr}^{\operatorname{Ker}\left(f_{s}\right)}$, the canonical projection from $A_{s}$ to $A_{s} / \operatorname{Ker}\left(f_{s}\right), f_{s}^{\mathrm{b}}$ the canonical isomorphism from $A_{s} / \operatorname{Ker}\left(f_{s}\right)$ to $\operatorname{Im}\left(f_{s}\right),\left(\operatorname{in}^{\operatorname{Im}(f)}\right)_{s}$ the canonical embedding $\operatorname{in} \operatorname{Im}\left(f_{s}\right)$ of $\operatorname{Im}\left(f_{s}\right)$ into $B_{s}$, $f_{s}^{\text {e the }}$ corestriction of $f_{s}$ to $\operatorname{Im}\left(f_{s}\right)$ and $f_{s}^{\mathrm{m}}$ the mapping which sends $[a]_{\operatorname{Ker}\left(f_{s}\right)}$ in $A_{s} / \operatorname{Ker}\left(f_{s}\right)$ to $f_{s}(a)$ in $B_{S}$.

We next define the concept of free monoid on a set and several notions associated with it that will be used afterwards to construct the free algebra on an $S$-sorted set.

Definition 12. Let $A$ be a set. The free monoid on $A$, denoted by $\mathbf{A}^{\star}$, is $\left(A^{\star}, \curlywedge, \lambda\right)$, where $A^{\star}$, the set of all words on $A$, is $\bigcup_{n \in \mathbb{N}} \operatorname{Hom}(n, A)$, the set of all mappings $w: n \longrightarrow A$ from some $n \in \mathbb{N}$ to $A$, ᄉ, the concatenation of words on $A$, is the binary operation on $A^{\star}$ which sends a pair 
of words $(w, v)$ on $A$ to the mapping $w \curlywedge v$ from $|w|+|v|$ to $A$, where $|w|$ and $|v|$ are the lengths (三 domains) of the mappings $w$ and $v$, respectively, defined as follows:

$$
w \curlywedge v\left\{\begin{array}{cl}
|w|+|v| & \longrightarrow S \\
i & \longmapsto \begin{cases}w_{i}, & \text { if } 0 \leq i<|w| ; \\
v_{i-|w|}, & \text { if }|w| \leq i<|w|+|v|,\end{cases}
\end{array}\right.
$$

and $\lambda$, the empty word on $A$, is the unique mapping from $\varnothing$ to $A$. A word $w \in A^{\star}$ is usually denoted as a sequence $\left(a_{i}\right)_{i \in|w|}$, where, for $i \in|w|, a_{i}$ is the letter in A satisfying $w(i)=a_{i}$. We will denote by $\eta_{A}$ the mapping from $A$ to $A^{\star}$ that sends $a \in A$ to $(a) \in A^{\star}$, i.e., to the mapping $(a): 1 \longrightarrow A$ that sends 0 to $a$. The ordered pair $\left(\mathbf{A}^{\star}, \eta_{A}\right)$ is a universal morphism from $A$ to the forgetful functor from the category Mon, of monoids, to Set.

\section{Many-sorted algebras}

Our next aim is to provide those notions from the field of many-sorted universal algebra that will be used afterwards. We will specially focus on the constructive description of the subalgebra generating many-sorted operator, the congruence generating many-sorted operator and the (absolutely) free many-sorted algebra on a many-sorted set.

Convention. In what follows, for a set of sorts $S$, an arbitrary word on $S^{\star}$ will be denoted by $\mathbf{s}$, i.e., a lower case bold type s. The letter s will be used to represent an arbitrary letter in $S$.

Definition 13. An $S$-sorted signature is a mapping $\Sigma$ from $S^{\star} \times S$ to $\mathcal{U}$ which sends a pair $(\mathbf{s}, s) \in S^{\star} \times S$ to the set $\Sigma_{\mathbf{s}, s}$ of the formal operations of arity s, sort (or coarity) s and rank (or biarity) $(\mathbf{s}, s)$.

Assumption 2. From now on $\Sigma$ stands for an S-sorted signature, fixed once and for all.

We shall now give precise definitions of the concepts of many-sorted algebra and homomorphism between many-sorted algebras.

Definition 14. The $S^{\star} \times S$-sorted set of the finitary operations on an $S$-sorted set $A$ is $\left(\operatorname{Hom}\left(A_{\mathbf{s}}, A_{s}\right)\right)_{(\mathbf{s}, s}$ where, for every $\mathbf{s} \in S^{\star}, A_{\mathbf{s}}=\prod_{j \in|\mathbf{s}|} A_{s_{j}}$, with $|\mathbf{s}|$ denoting the length of the word $\mathbf{s}$ (if $\mathbf{s}=\lambda$, then $A_{\lambda}$ is a final set). A structure of $\Sigma$-algebra on an $S$-sorted set $A$ is a family $\left(F_{\mathbf{s}, s}\right)_{(\mathbf{s}, s) \in S^{\star} \times S \text {, }}$ denoted by $F$, where, for $(\mathbf{s}, s) \in S^{\star} \times S, F_{\mathbf{s}, s}$ is a mapping from $\Sigma_{\mathbf{s}, s}$ to $\operatorname{Hom}\left(A_{\mathbf{s}}, A_{s}\right)$ (if $(\mathbf{s}, s)=(\lambda, s)$ and $\sigma \in \Sigma_{\lambda, s}$, then $F_{\lambda, s}(\sigma)$ picks out an element of $\left.A_{s}\right)$. For a pair $(\mathbf{s}, s) \in S^{\star} \times S$ and a formal operation $\sigma \in \Sigma_{\mathbf{s}, s}$, in order to simplify the notation, the operation $F_{\mathbf{s}, s}(\sigma)$ from $A_{\mathbf{s}}$ to $A_{s}$ will be written as $F_{\sigma}$. A many-sorted $\Sigma$-algebra (or, to abbreviate, $\Sigma$-algebra) is a pair $(A, F)$, denoted by $\mathbf{A}$, where $A$ is an $S$-sorted set and $F$ a structure of $\Sigma$-algebra on $A$. $A$ - - -homomorphism (or, to abbreviate, homomorphism) from $\mathbf{A}$ to $\mathbf{B}$, where $\mathbf{B}=(B, G)$, is a triple $(\mathbf{A}, f, \mathbf{B})$, denoted by $f: \mathbf{A} \longrightarrow \mathbf{B}$, where $f$ is an $S$-sorted mapping from $A$ to $B$ such that, for every $(\mathbf{s}, s) \in S^{\star} \times S$, every $\sigma \in \Sigma_{\mathbf{s}, s}$ and every $\left(a_{j}\right)_{j \in|\mathbf{s}|} \in A_{\mathbf{s}}$, we have $f_{s}\left(F_{\sigma}\left(\left(a_{j}\right)_{j \in|\mathbf{s}|}\right)\right)=G_{\sigma}\left(f_{\mathbf{s}}\left(\left(a_{j}\right)_{j \in|\mathbf{s}|}\right)\right)$, where $f_{\mathbf{s}}$ is the mapping $\prod_{j \in|\mathbf{s}|} f_{s_{j}}$ from $A_{\mathbf{s}}$ to $B_{\mathbf{s}}$ that sends $\left(a_{j}\right)_{j \in|\mathbf{s}|}$ in $A_{\mathbf{s}}$ to $\left(f_{s_{j}}\left(a_{j}\right)\right)_{j \in|\mathbf{s}|}$ in $B_{\mathbf{s}}$. We will denote by $\operatorname{Alg}(\Sigma)$ the category of $\Sigma$-algebras and homomorphisms and by $\operatorname{Alg}(\Sigma)$ the set of objects of $\operatorname{Alg}(\Sigma)$.

In some cases, to avoid mistakes, we will denote by $F^{\mathbf{A}}$ the structure of $\Sigma$-algebra on $A$, and, for $(\mathbf{s}, s) \in S^{\star} \times S$ and $\sigma \in \Sigma_{\mathbf{s}, s}$, by $F_{\sigma}^{\mathbf{A}}$, or simply by $\sigma^{\mathbf{A}}$, the corresponding operation. Moreover, for $s \in S$ and $\sigma \in \Sigma_{\lambda, s}$, we will, usually, denote by $\sigma^{\mathbf{A}}$ the value of the mapping $F_{\sigma}^{\mathbf{A}}: A_{\lambda} \longrightarrow A_{S}$ at the unique element in $A_{\lambda}$.

We will denote by $\mathbf{1}^{S}$ or, to abbreviate, by $\mathbf{1}$, the (standard) final $\Sigma$-algebra.

We add some further results concerning direct products and homomorphisms, which will be of great importance later on. 
Definition 15. Let $\left(\mathbf{A}^{i}\right)_{i \in I}$ be a family of $\Sigma$-algebras, where we agree that, for every $i \in I$, $\mathbf{A}^{i}=\left(A^{i}, F^{i}\right)$. Then

1. The product of $\left(\mathbf{A}^{i}\right)_{i \in I}, \prod_{i \in I} \mathbf{A}^{i}$, is the $\Sigma$-algebra which has as $S$-sorted underlying set $\prod_{i \in I} A^{i}$, and where, for every $(\mathbf{s}, s) \in S^{\star} \times S$ and every $\sigma \in \Sigma_{\mathbf{s}, s}$, the structural operation $F_{\sigma}$ is defined as follows:

$$
F_{\sigma}\left\{\begin{aligned}
\left(\prod_{i \in I} A^{i}\right)_{\mathbf{s}} & \longrightarrow \prod_{i \in I} A_{s}^{i} \\
\left(a_{j}\right)_{j \in|\mathbf{s}|} & \longmapsto\left(F_{\sigma}^{i}\left(\left(a_{j}(i)\right)_{j \in|\mathbf{s}|}\right)_{i \in I} .\right.
\end{aligned}\right.
$$

2. For every $i \in I$, the $i$-th canonical projection is the homomorphism from $\prod_{i \in I} \mathbf{A}^{i}$ to $\mathbf{A}^{i}$ determined by the S-sorted mapping $\mathrm{pr}^{i}$ which, for every $s \in S$, is defined as follows

$$
\operatorname{pr}_{s}^{i}\left\{\begin{aligned}
\prod_{i \in I} A_{s}^{i} & \longrightarrow A_{s}^{i} \\
\left(a_{i}\right)_{i \in I} & \longmapsto a_{i}
\end{aligned}\right.
$$

Proposition 6. Let $\left(\mathbf{A}^{i}\right)_{i \in I}$ be a family of $\Sigma$-algebras. Then the pair $\left(\prod_{i \in I} \mathbf{A}^{i},\left(\operatorname{pr}^{i}\right)_{i \in I}\right)$ is a product in $\operatorname{Alg}(\Sigma)$.

We next introduce the support of a many-sorted algebra and the definition of finiteness of a many-sorted algebra.

Definition 16. Let $\mathbf{A}$ be a $\Sigma$-algebra. Then the support of $\mathbf{A}$, denoted by $\operatorname{supp}_{S}(\mathbf{A})$, is $\operatorname{supp}_{S}(A)$, i.e., the support of the underlying $S$-sorted set $A$ of $\mathbf{A}$.

Remark 3. The set $\left\{\operatorname{supp}_{S}(\mathbf{A}) \mid \mathbf{A} \in \operatorname{Alg}(\Sigma)\right\}$ is a closure system on $S$.

Definition 17. Let $\mathbf{A}$ be a $\Sigma$-algebra. We will say that $\mathbf{A}$ is finite if $A$, the underlying $S$-sorted set of $\mathbf{A}$, is finite.

We shall now go on to define the notion of subalgebra of a $\Sigma$-algebra $\mathbf{A}$, the principle of proof by Algebraic Induction and the subalgebra generating operator for $\mathbf{A}$.

Definition 18. Let $\mathbf{A}=(A, F)$ be a $\Sigma$-algebra and $X \subseteq A$. Given $(\mathbf{s}, s) \in S^{\star} \times S$ and $\sigma \in \Sigma_{\mathbf{s}, s}$, we will say that $X$ is closed under the operation $F_{\sigma}: A_{\mathbf{s}} \longrightarrow A_{s}$ if, for every $\left(a_{j}\right)_{j \in|\mathbf{s}|} \in X_{\mathbf{s}}$, $F_{\sigma}\left(\left(a_{j}\right)_{j \in|\mathbf{s}|}\right) \in X_{s}$. We will say that $X$ is a closed subset of $\mathbf{A}$ if $X$ is closed under the operations of $\mathbf{A}$. We will denote by $\mathrm{Cl}(\mathbf{A})$ the set of all closed subsets of $\mathbf{A}$ (which is an algebraic closure system on $A)$ and by $\mathrm{Cl}(\mathbf{A})$ the algebraic lattice $(\mathrm{Cl}(\mathbf{A}), \subseteq)$. We will say that a $\Sigma$-algebra $\mathbf{B}$ is a subalgebra of $\mathbf{A}$ if $B \subseteq A$ and the canonical embedding of $B$ into $A$ determines an embedding of $\mathbf{B}$ into $\mathbf{A}$. We will denote by $\operatorname{Sub}(\mathbf{A})$ the set of all subalgebras of $\mathbf{A}$. Since $\mathrm{Cl}(\mathbf{A})$ and $\operatorname{Sub}(\mathbf{A})$ are isomorphic, we shall feel free to deal either with a closed subset of $\mathbf{A}$ or with the correlated subalgebra of $\mathbf{A}$, whichever is most convenient for the work at hand.

Definition 19. Let $\mathbf{A}$ be a $\Sigma$-algebra. Then we denote by $\mathrm{Sg}_{\mathbf{A}}$ the many-sorted closure operator on A defined as follows:

$$
\operatorname{Sg}_{\mathbf{A}}\left\{\begin{array}{cl}
\operatorname{Sub}(A) & \longrightarrow \operatorname{Sub}(A) \\
X & \longmapsto \cap\{C \in \operatorname{Sub}(\mathbf{A}) \mid X \subseteq C\}
\end{array}\right.
$$

We call $\mathrm{Sg}_{\mathrm{A}}$ the subalgebra generating many-sorted operator on $A$ determined by $\mathbf{A}$. For every $X \subseteq A$, we call $\mathrm{Sg}_{\mathbf{A}}(X)$ the subalgebra of $\mathbf{A}$ generated by $X$. Moreover, if $X \subseteq A$ is such that $\operatorname{Sg}_{\mathbf{A}}(X)=A$, then we say that $X$ is an $S$-sorted set of generators of $\mathbf{A}$, or that $X$ generates $\mathbf{A}$. Besides, we say that $\mathbf{A}$ is finitely generated if there exists an $S$-sorted subset $X$ of $A$ such that $X$ generates $\mathbf{A}$ and $\operatorname{card}(X)<\aleph_{0}$. 
Remark 4. Let $\mathbf{A}$ be a $\sum$-algebra. Then the algebraic closure operator $\mathrm{Sg}_{\mathbf{A}}$ is uniform, i.e., for every $X, Y \subseteq A$, if $\operatorname{supp}_{S}(X)=\operatorname{supp}_{S}(Y)$, then we have $\operatorname{supp}_{S}\left(\operatorname{Sg}_{\mathbf{A}}(X)\right)=\operatorname{supp}_{S}\left(\operatorname{Sg}_{\mathbf{A}}(Y)\right)$.

We next recall the Principle of Proof by Algebraic Induction.

Proposition 7 (Principle of Proof by Algebraic Induction). Let $\mathbf{A}$ be a E-algebra generated by $X$. Then to prove that a subset $Y$ of $A$ is equal to $A$ it suffices to show:

1. $X \subseteq Y$ (algebraic induction basis); and

2. $Y$ is a subalgebra of $\mathbf{A}$ (algebraic induction step).

Proposition 8. Let A be a $\sum$-algebra. Then the many-sorted closure operator $\mathrm{Sg}_{\mathbf{A}}$ on $A$ is algebraic, i.e., for every $S$-sorted subset $X$ of $A, \operatorname{Sg}_{\mathbf{A}}(X)=\bigcup_{K \subseteq_{\mathrm{f}} X} \operatorname{Sg}_{\mathbf{A}}(K)$.

For a $\Sigma$-algebra A we next provide another, more constructive, description of the algebraic many-sorted closure operator $\mathrm{Sg}_{\mathbf{A}}$.

Definition 20. Let $\Sigma$ be an S-sorted signature and $\mathbf{A}$ a $\Sigma$-algebra.

1. We denote by $\mathrm{E}_{\mathbf{A}}$ the many-sorted operator on $A$ that assigns to an $S$-sorted subset $X$ of $A$, $\mathrm{E}_{\mathbf{A}}(X)=X \cup\left(\bigcup_{\sigma \in \Sigma_{\neq \lambda, s}} F_{\sigma}\left[X_{\operatorname{ar}(\sigma)}\right]\right)_{s \in S^{\prime}}$ where, for $s \in S, \Sigma_{\neq \lambda, s}$ is the set of all manysorted formal operations $\sigma$ such that $\operatorname{ar}(\sigma) \in S^{\star}-\{\lambda\}, \operatorname{car}(\sigma)=s$, and if $\operatorname{ar}(\sigma)=\mathbf{s}$, then $X_{\operatorname{ar}(\sigma)}=\prod_{i \in|\mathbf{s}|} X_{s_{i}}$.

2. If $X \subseteq A$, then we define the family $\left(\mathrm{E}_{\mathbf{A}}^{n}(X)\right)_{n \in \mathbb{N}}$ in $\operatorname{Sub}(A)$, recursively, as follows:

$$
\begin{aligned}
\mathrm{E}_{\mathbf{A}}^{0}(X) & =X \cup\left(\cup_{\sigma \in \Sigma_{\lambda, s}} F_{\sigma}\left[A_{\lambda}\right]\right)_{s \in S}, \\
\mathrm{E}_{\mathbf{A}}^{n+1}(X) & =\mathrm{E}_{\mathbf{A}}\left(\mathrm{E}_{\mathbf{A}}^{n}(X)\right), n \geq 0 .
\end{aligned}
$$

3. We denote by $\mathrm{E}_{\mathbf{A}}^{\omega}$ the many-sorted operator on $A$ that assigns to an S-sorted subset $X$ of $A$, $\mathrm{E}_{\mathbf{A}}^{\omega}(X)=\bigcup_{n \in \mathbb{N}} \mathrm{E}_{\mathbf{A}}^{n}(X)$.

Remark 5. Since, for $s \in S$ and $\sigma \in \Sigma_{\lambda, s}, \sigma^{\mathbf{A}}$ is the value of $F_{\sigma}^{\mathbf{A}}: A_{\lambda} \longrightarrow A_{S}$ at the unique element in $A_{\lambda}$, it follows that

$$
\left(\bigcup_{\sigma \in \Sigma_{\lambda, s}} F_{\sigma}\left[A_{\lambda}\right]\right)_{s \in S}=\left(\left\{\sigma^{\mathbf{A}} \mid \sigma \in \Sigma_{\lambda, s}\right\}\right)_{s \in S}
$$

Proposition 9. Let $\mathbf{A}$ be a $\Sigma$-algebra and $X \subseteq A$, then $\operatorname{Sg}_{\mathbf{A}}(X)=\mathrm{E}_{\mathbf{A}}^{\omega}(X)$.

Remark 6. For a homomorphism $f$ from a $\sum$-algebra $\mathbf{A}$ to another $\mathbf{B}$ and a subset $X$ of $A$, we have that $f\left[\operatorname{Sg}_{\mathbf{A}}(X)\right] \subseteq \operatorname{Sg}_{\mathbf{B}}(f[X])$.

Our next goal is to define the concepts of congruence on a $\Sigma$-algebra and of quotient of a $\Sigma$-algebra by a congruence on it. Moreover, we recall the notion of kernel of a homomorphism between $\Sigma$-algebras, the universal property of the quotient of a $\Sigma$-algebra by a congruence on it and the congruence generating operator for $\mathbf{A}$.

Definition 21. Let $\mathbf{A}$ be a $\sum$-algebra and $\Phi$ an S-sorted equivalence on $A$. We will say that $\Phi$ is an $S$-sorted congruence on (or, to abbreviate, a congruence on) $\mathbf{A}$ if, for every $(\mathbf{s}, s) \in$ $\left(S^{\star}-\{\lambda\}\right) \times S$, every $\sigma \in \Sigma_{\mathbf{s}, s}$, and every $\left(a_{j}\right)_{j \in|\mathbf{s}|},\left(b_{j}\right)_{j \in|\mathbf{s}|} \in A_{\mathbf{s}}$, if, for every $j \in|\mathbf{s}|,\left(a_{j}, b_{j}\right) \in$ $\Phi_{s_{j}}$, then $\left(F_{\sigma}\left(\left(a_{j}\right)_{j \in|\mathbf{s}|}\right), F_{\sigma}\left(\left(b_{j}\right)_{j \in|\mathbf{s}|}\right)\right) \in \Phi_{s}$. We will denote by $\operatorname{Cgr}(\mathbf{A})$ the set of all $S$-sorted congruences on $\mathbf{A}$ (which is an algebraic closure system on $A \times A)$, by $\mathbf{C g r}(\mathbf{A})$ the algebraic lattice $(\operatorname{Cgr}(\mathbf{A}), \subseteq)$, by $\nabla_{\mathbf{A}}$ the greatest element of $\mathbf{C g r}(\mathbf{A})$ and by $\Delta_{\mathbf{A}}$ the least element of $\mathbf{C g r}(\mathbf{A})$.

For a congruence $\Phi$ on $\mathbf{A}$, the quotient $\Sigma$-algebra of $\mathbf{A}$ by $\Phi$, denoted by $\mathbf{A} / \Phi$, is the $\Sigma$-algebra $\left(A / \Phi, F^{\mathbf{A} / \Phi}\right)$, where, for every $(\mathbf{s}, s) \in S^{\star} \times S$ and every $\sigma \in \Sigma_{\mathbf{s}, s}$, the operation $F_{\sigma}^{\mathbf{A}} / \Phi$ from $(A / \Phi)_{w}$ to $A_{s} / \Phi_{s}$, also denoted, to simplify, by $F_{\sigma}$, sends $\left(\left[a_{j}\right]_{\Phi_{s_{j}}}\right)_{j \in|\mathbf{s}|}$ in $(A / \Phi)_{\mathbf{s}}$ to $\left[F_{\sigma}\left(\left(a_{j}\right)_{j \in|\mathbf{s}|}\right)\right]_{\Phi_{s}}$ in $A_{s} / \Phi_{s}$, and the canonical projection from $\mathbf{A}$ to $\mathbf{A} / \Phi$, denoted 
by $\operatorname{pr}^{\Phi}: \mathbf{A} \longrightarrow \mathbf{A} / \Phi$, is the full surjective homomorphism determined by the projection from $A$ to $A / \Phi$. The ordered pair $\left(\mathbf{A} / \Phi, \mathrm{pr}^{\Phi}\right)$ has the following universal property: $\operatorname{Ker}\left(\mathrm{pr}^{\Phi}\right)$ is $\Phi$ and, for every $\Sigma$-algebra $\mathbf{B}$ and every homomorphism from $\mathbf{A}$ to $\mathbf{B}$, if $\operatorname{Ker}(f) \supseteq \Phi$, then there exists a unique homomorphism $h$ from $\mathbf{A} / \Phi$ to $\mathbf{B}$ such that $h \circ \mathrm{pr}^{\Phi}=f$. In particular, if $\Psi$ is a congruence on $A$ such that $\Phi \subseteq \Psi$, then we will denote by $\mathrm{p}^{\Phi, \Psi}$ the unique homomorphism from $\mathbf{A} / \Phi$ to $\mathbf{A} / \Psi$ such that $\mathrm{p}^{\Phi, \Psi} \circ \mathrm{pr}^{\Phi}=\mathrm{pr}^{\Psi}$.

Remark 7. Let $\mathbf{C l f d A l g}(\Sigma)$ be the category whose objects are the classified $\Sigma$-algebras, i.e, the ordered pairs $(\mathbf{A}, \Phi)$ where $\mathbf{A}$ is a $\sum$-algebra and $\Phi$ a congruence on $\mathbf{A}$, and in which the set of morphisms from $(\mathbf{A}, \Phi)$ to $(\mathbf{B}, \Psi)$ is the set of all homomorphisms $f$ from $\mathbf{A}$ to $\mathbf{B}$ such that, for every $s \in S$ and every $(x, y) \in A_{s}^{2}$, if $(x, y) \in \Phi_{s}$, then $\left(f_{s}(x), f_{s}(y)\right) \in \Psi_{s}$. Let $G$ be the functor from $\mathbf{A} \lg (\Sigma)$ to $\mathbf{C l f d} \mathbf{A} \lg (\Sigma)$ whose object mapping sends $\mathbf{A}$ to $\left(\mathbf{A}, \Delta_{\mathbf{A}}\right)$ and whose morphism mapping sends $f: \mathbf{A} \longrightarrow \mathbf{B}$ to $f:\left(\mathbf{A}, \Delta_{\mathbf{A}}\right) \longrightarrow\left(\mathbf{B}, \Delta_{\mathbf{B}}\right)$. Then, for every classified $\Sigma$-algebra $(\mathbf{A}, \Phi)$, there exists a universal mapping from $(\mathbf{A}, \Phi)$ to $G$, which is precisely the ordered pair $\left(\mathbf{A} / \Phi, \mathrm{pr}^{\Phi}\right)$ with $\operatorname{pr}^{\Phi}:(\mathbf{A}, \Phi) \longrightarrow\left(\mathbf{A} / \Phi, \Delta_{\mathbf{A} / \Phi}\right)$.

Definition 22. Let $\mathbf{A}$ be a $\Sigma$-algebra. Then we denote by $\mathrm{Cg}_{\mathbf{A}}$ the many-sorted closure operator on $A \times A$ defined as follows:

$$
\operatorname{Cg}_{\mathbf{A}}\left\{\begin{array}{cl}
\operatorname{Sub}(A \times A) & \longrightarrow \operatorname{Sub}(A \times A) \\
\Phi & \longmapsto \bigcap\{\Psi \in \operatorname{Con}(\mathbf{A}) \mid \Phi \subseteq \Psi\}
\end{array}\right.
$$

We call $\mathrm{Cg}_{\mathrm{A}}$ the congruence generating many-sorted operator on $A \times A$ determined by A. For every relation $\Phi \subseteq A \times A$, we call $\mathrm{Cg}_{\mathrm{A}}(\Phi)$ the congruence on $\mathbf{A}$ generated by $\Phi$.

For a $\Sigma$-algebra A we next provide another, more constructive, description of the algebraic many-sorted closure operator $\mathrm{Cg}_{\mathbf{A}}$.

Definition 23. Let $\Sigma$ be an S-sorted signature and $\mathbf{A}$ a $\Sigma$-algebra.

(1) We denote by $\mathrm{C}_{\mathbf{A}}$ the many-sorted operator on $A \times A$ that assigns to an $S$-sorted relation $\Phi \subseteq A \times A$, the $S$-sorted relation

$$
\mathrm{C}_{\mathbf{A}}(\Phi)=(\Phi \circ \Phi) \cup\left(\bigcup_{\sigma \in \Sigma_{\neq \lambda, s}} F_{\sigma} \times F_{\sigma}\left[\Phi_{\operatorname{ar}(\sigma)}\right]\right)_{s \in S^{\prime}}
$$

where, for $s \in S, \Sigma_{\neq \lambda, s}$ is the set of all many-sorted formal operations $\sigma$ such that $\operatorname{ar}(\sigma) \in$ $S^{\star}-\{\lambda\}, \operatorname{car}(\sigma)=s$ and if $\operatorname{ar}(\sigma)=\mathbf{s}$, then $\Phi_{\operatorname{ar}(\sigma)}=\prod_{j \in|\mathbf{s}|} \Phi_{s_{j}}$. Let us note that

$$
\Phi_{\operatorname{ar}(\sigma)} \subseteq \prod_{j \in|\mathbf{s}|} A_{s_{j}}^{2} \cong\left(\prod_{j \in|\mathbf{s}|} A_{s_{j}}\right)^{2}
$$

(2) If $\Phi \subseteq A \times A$, then we define the family $\left(\mathrm{C}_{\mathbf{A}}^{n}(\Phi)\right)_{n \in \mathbb{N}}$ in $\operatorname{Sub}(A \times A)$, recursively, as follows:

$$
\begin{aligned}
\mathrm{C}_{\mathbf{A}}^{0}(\Phi) & =\Phi \cup \Phi^{-1} \cup \Delta_{\mathrm{A}}, \\
\mathrm{C}_{\mathbf{A}}^{n+1}(\Phi) & =\mathrm{C}_{\mathbf{A}}\left(\mathrm{C}_{\mathbf{A}}^{n}(\Phi)\right), n \geq 0 .
\end{aligned}
$$

(3) We denote by $\mathrm{C}_{\mathbf{A}}^{\omega}$ the many-sorted operator on $A \times A$ that assigns to an $S$-sorted relation $\Phi \subseteq A \times A, C_{\mathbf{A}}^{\omega}(\Phi)=\bigcup_{n \in \mathbb{N}} C_{\mathbf{A}}^{n}(\Phi)$

Proposition 10. Let $\mathbf{A}$ be a $\Sigma$-algebra and let $\Phi \subseteq A \times A$ be a relation on $A$. For every $n \in \mathbb{N}$ we have that

(1) $C_{\mathbf{A}}^{n}(\Phi)$ is a reflexive relation;

(2) $\mathrm{C}_{\mathbf{A}}^{n}(\Phi) \subseteq \mathrm{C}_{\mathbf{A}}^{n+1}(\Phi)$;

(3) $C_{\mathbf{A}}^{n}(\Phi)$ is a symmetric relation. 
Proposition 11. Let $\mathbf{A}$ be a $\sum$-algebra and $\Phi \subseteq A \times A$, then $\operatorname{Cg}_{\mathbf{A}}(\Phi)=\mathrm{C}_{\mathbf{A}}^{\omega}(\Phi)$.

We next state that the forgetful functor $G_{\Sigma}$ from $\operatorname{Alg}(\Sigma)$ to $\operatorname{Set}^{S}$ has a left adjoint $\mathbf{T}_{\Sigma}$ which assigns to an $S$-sorted set $X$ the free $\Sigma$-algebra $\mathbf{T}_{\Sigma}(X)$ on $X$. We latter state the universal property of the free many-sorted algebra and provide a characterization by means of the notion of Dedekind Peano-algebras.

Let us note that in what follows, to construct the algebra of $\Sigma$-rows in $X$, and the free $\Sigma$-algebra on $X$, since neither the $S$-sorted signature $\Sigma$ nor the $S$-sorted set $X$ are subject to any constraint, coproducts must necessarily be used.

Definition 24. Let $X$ be an $S$-sorted set. The algebra of $\Sigma$-rows in $X$, denoted by $\mathbf{W}_{\Sigma}(X)$, is defined as follows:

1. The underlying $S$-sorted set of $\mathbf{W}_{\Sigma}(X)$, written as $\mathrm{W}_{\Sigma}(X)$, is precisely the $S$-sorted set $\left((\amalg \Sigma \amalg \amalg X)^{\star}\right)_{s \in S}$, i.e., the mapping from $S$ to $\mathbf{U}$ constantly $(\amalg \Sigma \amalg \amalg X)^{\star}$, where $(\amalg \Sigma \amalg$ $\amalg X)^{\star}$ is the set of all words on the set $\amalg \Sigma \amalg \amalg X$, i.e., on the set

$$
\left[\left(\bigcup_{(\mathbf{s}, s) \in S^{\star} \times S}\left(\Sigma_{\mathbf{s}, s} \times\{(\mathbf{s}, s)\}\right)\right) \times\{0\}\right] \cup\left[\left(\bigcup_{s \in S}\left(X_{S} \times\{s\}\right)\right) \times\{1\}\right] .
$$

2. For every $(\mathbf{s}, s) \in S^{\star} \times S$ and every $\sigma \in \Sigma_{\mathbf{s}, s}$, the structural operation $F_{\sigma}$ associated to $\sigma$ is the mapping from $\mathrm{W}_{\Sigma}(X)_{\mathbf{s}}$ to $\mathrm{W}_{\Sigma}(X)_{s}$ which sends $\left(P_{j}\right)_{j \in|\mathbf{s}|} \in \mathrm{W}_{\Sigma}(X)_{\mathbf{s}}$ to $(\sigma) \curlywedge_{\boldsymbol{N} \in|\mathbf{s}|} P_{j} \in$ $\mathrm{W}_{\Sigma}(X)_{s^{\prime}}$ where, for every $(\mathbf{s}, s) \in S^{\star} \times S$ and every $\sigma \in \Sigma_{\mathbf{s}, s},(\sigma)$ stands for $(((\sigma,(\mathbf{s}, s)), 0))$, which is the value at $\sigma$ of the canonical mapping from $\Sigma_{\mathbf{s}, s}$ to $(\amalg \Sigma \amalg \amalg X)^{\star}$.

Definition 25. The free $\Sigma$-algebra on an $S$-sorted set $X$, denoted by $\mathbf{T}_{\Sigma}(X)$, is the $\Sigma$-algebra determined by $\operatorname{Sg}_{\mathbf{W}_{\Sigma}(X)}\left(\left(\left\{(x) \mid x \in X_{S}\right\}\right)_{s \in S}\right)$, the subalgebra of $\mathbf{W}_{\Sigma}(X)$ generated by $(\{(x) \mid$ $\left.\left.x \in X_{s}\right\}\right)_{s \in S}$, where, for every $s \in S$ and every $x \in X_{s},(x)$ stands for $(((x, s), 1))$, which is the value at $x$ of the canonical mapping from $X_{S}$ to $(\amalg \Sigma \amalg \amalg X)^{\star}$. We will denote by $\mathrm{T}_{\Sigma}(X)$ the underlying $S$-sorted of $\mathbf{T}_{\Sigma}(X)$ and, for $s \in S$, we will call the elements of $\mathrm{T}_{\Sigma}(X)_{s}$ terms of type $s$ with variables in $X$ or $(X, s)$-terms.

Remark 8. Since $\left(\left\{(x) \mid x \in X_{S}\right\}\right)_{s \in S}$ is a generating subset of $\mathbf{T}_{\Sigma}(X)$, to prove that a subset $\mathscr{T}$ of $\mathrm{T}_{\Sigma}(X)$ is equal to $\mathrm{T}_{\Sigma}(X)$ it suffices, by Proposition 21, to show:

1. $\left(\left\{(x) \mid x \in X_{S}\right\}\right)_{s \in S} \subseteq \mathscr{T}$ (algebraic induction basis); and

2. $\mathscr{T}$ is a subalgebra of $\mathbf{T}_{\Sigma}(X)$ (algebraic induction step).

In the many-sorted case we have, as in the single-sorted case, the following characterization of the elements of $\mathrm{T}_{\Sigma}(X)_{s}$, for $s \in S$.

Proposition 12. Let $X$ be an $S$-sorted set. Then, for every $s \in S$ and every $P \in \mathrm{W}_{\Sigma}(X)_{s}$, we have that $P$ is a term of type s with variables in $X$ if and only if $P=(x)$, for a unique $x \in X_{s}$, or $P=(\sigma)$, for a unique $\sigma \in \Sigma_{\lambda, s}$,or $P=(\sigma) \curlywedge \curlywedge\left(P_{j}\right)_{j|\mathbf{s}|}$, for a unique $\mathbf{s} \in S^{\star}-\{\lambda\}$, a unique $\sigma \in \Sigma_{\mathbf{s}, s}$ and a unique family $\left(P_{j}\right)_{j \in|\mathbf{s}|} \in \mathrm{T}_{\Sigma}(X)_{\mathbf{s}}$. Moreover, the three possibilities are mutually exclusive. From now on, for simplicity of notation, we will write $x, \sigma$ and $\sigma\left(P_{0}, \ldots, P_{|\mathbf{s}|-1}\right)$ or $\sigma\left(\left(P_{j}\right)_{j \in|\mathbf{s}|}\right)$ instead of $(x),(\sigma)$ and $(\sigma) \curlywedge \curlywedge\left(P_{j}\right)_{j \in|\mathbf{s}|}$, respectively. Thus, in particular, the structural operation $F_{\sigma}$ (or, more accurately, $F_{\sigma}^{\mathbf{T}_{\Sigma}(X)}$ ) associated to $\sigma$ is identified with $\sigma$.

From the above proposition it follows, immediately, the universal property of the free $\Sigma$-algebra on an $S$-sorted set $X$, as stated in the subsequent proposition.

Proposition 13. For every S-sorted set $X$, the pair $\left(\eta_{X}, \mathbf{T}_{\Sigma}(X)\right)$, where $\eta^{X}$, the insertion of (the $S$-sorted set of generators) $X$ into $\mathrm{T}_{\Sigma}(X)$, is the co-restriction to $\mathrm{T}_{\Sigma}(X)$ of the canonical embedding of $X$ into $\mathrm{W}_{\Sigma}(X)$, has the following universal property: for every $\Sigma$-algebra $\mathbf{A}$ and every S-sorted mapping $f: X \longrightarrow A$, there exists a unique homomorphism $f^{\sharp}: \mathbf{T}_{\Sigma}(X) \longrightarrow \mathbf{A}$ such that $f^{\sharp} \circ \eta_{X}=f$. 
Proof. For every $s \in S$ and every $(X, s)$-term $P$, the $s$-th coordinate $f_{s}^{\sharp}$ of $f^{\sharp}$ is defined recursively as follows: $f_{s}^{\sharp}(x)=f_{s}(x)$, if $P=x$; $f_{s}^{\sharp}(\sigma)=\sigma$, if $P=\sigma$; and, finally, $f_{s}^{\sharp}\left(\sigma\left(P_{j}\right)_{j \in|\mathbf{s}|}\right)=F_{\sigma}\left(\left(f_{s_{j}}^{\sharp}\left(P_{j}\right)\right)_{j \in|\mathbf{s}|}\right)$, if $P=\sigma\left(\left(P_{j}\right)_{j \in|\mathbf{s}|}\right)$.

The just stated proposition allows us to carry out definitions by algebraic recursion on a free many-sorted algebra as indeed we will be doing throughout this paper.

Corollary 1. The functor $\mathbf{T}_{\Sigma}$, which sends an $S$-sorted set $X$ to $\mathbf{T}_{\Sigma}(X)$ and an S-sorted mapping $f$ from $X$ to $Y$ to $f^{@}\left(=\left(\eta_{Y} \circ f\right)^{\sharp}\right)$, the unique homomorphism from $\mathbf{T}_{\Sigma}(X)$ to $\mathbf{T}_{\Sigma}(Y)$ such that $f^{@} \circ \eta_{X}=\eta_{Y} \circ f$, is left adjoint for the forgetful functor $\mathrm{G}_{\Sigma}$ from $\mathbf{A l g}(\Sigma)$ to $\mathbf{S e t}^{S}$.

It is possible to give an internal characterization of the free algebras by means of the Dedekind-Peano algebras.

Definition 26. Let A be a $\Sigma$-algebra. We will say that $\mathbf{A}$ is a Dedekind-Peano $\Sigma$-algebra, abbreviated to DP $\Sigma$-algebra when this is unlikely to cause confusion, if the following axioms hold

DP1. For every $(\mathbf{s}, s) \in S^{\star} \times S$ and every $\sigma \in \Sigma_{\mathbf{s}, s}, F_{\sigma}: A_{\mathbf{s}} \longrightarrow A_{s}$ is injective.

$D P 2$. For every $s \in S$ and every $\sigma, \tau \in \Sigma$., s, if $\sigma \neq \tau$, then $\operatorname{Im}\left(F_{\sigma}\right) \cap \operatorname{Im}\left(F_{\tau}\right)=\varnothing$.

DP3. $\mathrm{Sg}_{\mathbf{A}}\left(A-\left(\bigcup_{\sigma \in \Sigma, s} \operatorname{Im}\left(F_{\sigma}\right)\right)_{s \in S}\right)=A$.

We call the $S$-sorted set $A-\left(\bigcup_{\sigma \in \Sigma_{, S}} \operatorname{Im}\left(F_{\sigma}\right)\right)_{s \in S}$ the basis of Dedekind-Peano of $\mathbf{A}$, and we denote it by $\mathrm{B}(\mathbf{A})$.

We remark that the axioms DP1 and DP2 are equivalent to the following axiom

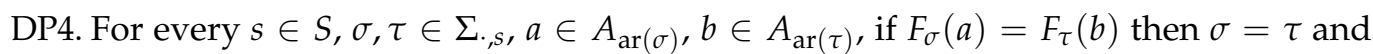
$a=b$.

Moreover, if $\mathbf{A}$ is a DP $\sum$-algebra, then $\mathrm{B}(\mathbf{A})$ is $\bigcap\left\{X \subseteq A \mid \mathrm{Sg}_{\mathbf{A}}(X)=A\right\}$

Proposition 14. Let $\mathbf{A}$ be a DP E-algebra. Then $\mathbf{A}$ is isomorphic to $\mathbf{T}_{\Sigma}(\mathbf{B}(\mathbf{A}))$.

Proposition 15. Let X be an S-sorted set. Then $\mathbf{T}_{\Sigma}(X)$ is a DP E-algebra.

\section{Many-sorted partial algebras}

In this section we gather together the basic facts about many-sorted partial algebras that we will need afterwards. Specifically, we define the notion of many-sorted partial algebra, we introduce the homomorphisms between many-sorted partial algebra and several classes of them, as e.g., the closed and the full homomorphisms. Moreover, we define, in connection with the homomorphisms and their properties, several classes of subobjects and quotient objects of the many-sorted partial algebras.

Definition 27. A structure of partial $\Sigma$-algebra on an $S$-sorted set $A$ is a family $\left(F_{\mathbf{s}, s}\right)_{(\mathbf{s}, s) \in S^{\star} \times S^{\prime}}$ denoted by $F$, where, for $(\mathbf{s}, s) \in S^{\star} \times S, F_{\mathbf{s}, s}$ is a mapping from $\Sigma_{\mathbf{s}, s}$ to $\operatorname{Hom}_{\mathrm{p}}\left(A_{\mathbf{s}}, A_{s}\right)$. If $\operatorname{Dom}\left(F_{\mathbf{s}, s}(\sigma)\right)=A_{\mathbf{s}}$, then $F_{\mathbf{s}, s}(\sigma)$ is a total operation from $A_{\mathbf{s}}$ to $A_{s}$. If $\operatorname{Dom}\left(F_{\mathbf{s}, s}(\sigma)\right)=\varnothing$, then $F_{\mathbf{s}, s}(\sigma)$ is called a discrete operation or an empty (partial) operation from $A_{\mathbf{s}}$ to $A_{s}$. If $(\mathbf{s}, s)=(\lambda, s), \sigma \in \Sigma_{\lambda, s}$ and $\operatorname{Dom}\left(F_{\lambda, s}(\sigma)\right) \neq \varnothing$, then $F_{\lambda, s}(\sigma)$ picks out an element of $A_{s}$. Therefore a partial operation $F_{\lambda, s}(\sigma)$ from $A_{\lambda}$ to $A_{s}$, also called a partial constant $F_{\lambda, s}(\sigma)$ from $A_{\lambda}$ to $A_{s}$, is either discrete or distinguishes exactly an element of $A_{s}$. For a pair $(\mathbf{s}, s) \in S^{\star} \times S$ and $a$ formal operation $\sigma \in \Sigma_{\mathbf{s}, s}$, in order to simplify the notation, the partial operation $F_{\mathbf{s}, s}(\sigma)$ from $A_{\mathbf{s}}$ to $A_{s}$ will be written as $F_{\sigma}$. A many-sorted partial $\Sigma$-algebra (or, to abbreviate, partial $\Sigma$-algebra) is a pair $(A, F)$, denoted by $\mathbf{A}$, where $A$ is an $S$-sorted set and $F$ a structure of partial $\Sigma$-algebra on A. $A$ E-homomorphism (or, to abbreviate, homomorphism) from $\mathbf{A}$ to $\mathbf{B}$, where $\mathbf{B}=(B, G)$, is a triple $(\mathbf{A}, f, \mathbf{B})$, denoted by $f: \mathbf{A} \longrightarrow \mathbf{B}$, where $f$ is an $S$-sorted mapping from $A$ to $B$ such that, for every $(\mathbf{s}, s) \in S^{\star} \times S$, every $\sigma \in \Sigma_{\mathbf{s}, s}$ and every $\left(a_{j}\right)_{j \in|\mathbf{s}|} \in A_{\mathbf{s}}$, if $\left(a_{j}\right)_{j \in|\mathbf{s}|} \in \operatorname{Dom}\left(F_{\sigma}\right)$, then $\left(f_{s_{j}}\left(a_{j}\right)\right)_{j \in|\mathbf{s}|} \in \operatorname{Dom}\left(G_{\sigma}\right)$ and $f_{s}\left(F_{\sigma}\left(\left(a_{j}\right)_{j \in|\mathbf{s}|}\right)\right)=G_{\sigma}\left(f_{\mathbf{s}}\left(\left(a_{j}\right)_{j \in|\mathbf{s}|}\right)\right)$. We will denote by 
$\operatorname{PA} \lg (\Sigma)$ the category of partial $\Sigma$-algebras and homomorphisms and by $\operatorname{PAlg}(\Sigma)$ the set of objects of $\operatorname{PAlg}(\Sigma)$.

Remark 9. The forgetful functor from the category $\operatorname{PAlg}(\Sigma)$ to the category Set $^{S}$ has a left adjoint, denoted by $\mathbf{D}_{\Sigma}$, which sends an $S$-sorted set $A$ to $\mathbf{D}_{\Sigma}(A)$, the discrete partial $\Sigma$-algebra on $A$, defined as follows: Its underlying $S$-sorted set is $A$, and, for every $(\mathbf{s}, s) \in S^{\star} \times S$ and every $\sigma \in \Sigma_{\mathbf{s}, s}, \operatorname{Dom}\left(F_{\sigma}\right)=\varnothing$.

Remark 10. The category $\operatorname{Alg}(\Sigma)$ is a full subcategory of the category $\operatorname{PAlg}(\Sigma)$. We shall prove later that the canonical full embedding of $\operatorname{Alg}(\Sigma)$ into $\operatorname{PA} \lg (\Sigma)$ has a left adjoint, the free completion of a partial $\Sigma$-algebra, which will play a key role in this paper.

The following is a useful characterization of the isomorphisms (that is (partially) analogous to that of the homeomorphisms, i.e., bijective and bicontinuous applications between topological spaces). Later we will include additional characterizations of the isomorphisms by using the notions of closed homomorphism and of full homomorphism.

Proposition 16. Let $f$ be a homomorphism from $\mathbf{A}$ to $\mathbf{B}$. Then the following statements are equivalent:

1. $f$ is an isomorphism from $\mathbf{A}$ to $\mathbf{B}$, i.e., $f$ is a section and a retraction.

2. $f$ is bijective and $\left(\mathbf{B}, f^{-1}, \mathbf{A}\right)$, denoted by $f^{-1}: \mathbf{B} \longrightarrow \mathbf{A}$, is also a homomorphism from $\mathbf{B}$ to A.

Remark 11. Let $\mathbf{A}$ be a partial $\Sigma$-algebra. Then $\left(\mathbf{D}_{\Sigma}(A), \mathrm{id}_{A}, \mathbf{A}\right)$ is a bijective homomorphism, but it is an isomorphism if, and only if, $\mathbf{A}$ is discrete, too.

To establish some of the results that follow, it is useful to have the concept of product of a family of many-sorted partial algebras.

Definition 28. Let $\left(\mathbf{A}^{i}\right)_{i \in I}$ be a family of partial $\Sigma$-algebras, where we agree that, for every $i \in I$, $\mathbf{A}^{i}=\left(A^{i}, F^{i}\right)$. Then

1. The product of $\left(\mathbf{A}^{i}\right)_{i \in I}, \prod_{i \in I} \mathbf{A}^{i}$, is the partial $\Sigma$-algebra which has as $S$-sorted underlying set $\prod_{i \in I} A^{i}$, and where, for every $(\mathbf{s}, s) \in S^{\star} \times S$ and every $\sigma \in \Sigma_{\mathbf{s}, s}$, the structural operation $F_{\sigma}$ is defined as follows:

(a) $\operatorname{Dom}\left(F_{\sigma}\right)=\left\{\left(a_{j}\right)_{j \in|\mathbf{s}|} \in\left(\prod_{i \in I} A^{i}\right)_{\mathbf{s}} \mid \forall i \in I\left(\left(a_{j}(i)\right)_{j \in|\mathbf{s}|} \in \operatorname{Dom}\left(F_{\sigma}^{i}\right)\right)\right\}$.

(b)

$$
F_{\sigma}\left\{\begin{aligned}
\operatorname{Dom}\left(F_{\sigma}\right) & \longrightarrow \prod_{i \in I} A_{s}^{i} \\
\left(a_{j}\right)_{j \in|\mathbf{s}|} & \longmapsto\left(F_{\sigma}^{i}\left(\left(a_{j}(i)\right)_{j \in|\mathbf{s}|}\right)_{i \in I}\right.
\end{aligned}\right.
$$

2. For every $i \in I$, the $i$-th canonical projection is the homomorphism from $\prod_{i \in I} \mathbf{A}^{i}$ to $\mathbf{A}^{i}$ determined by the $S$-sorted mapping $\mathrm{pr}^{i}$ which, for every $s \in S$, is defined as follows

$$
\operatorname{pr}_{s}^{i}\left\{\begin{aligned}
\prod_{i \in I} A_{s}^{i} & \longrightarrow A_{s}^{i} \\
\left(a_{i}\right)_{i \in I} & \longmapsto a_{i}
\end{aligned}\right.
$$

Proposition 17. Let $\left(\mathbf{A}^{i}\right)_{i \in I}$ be a family of partial $\Sigma$-algebras. Then the pair $\left(\prod_{i \in I} \mathbf{A}^{i},\left(\operatorname{pr}^{i}\right)_{i \in I}\right)$ is a product in $\operatorname{PAlg}(\Sigma)$.

In what follows we define the notions of subalgebra, relative subalgebra and weak subalgebra of a partial $\Sigma$-algebra $\mathbf{A}$ and the subalgebra generating operator for A. Furthermore, we characterize the subalgebras, the relative subalgebras and the weak subalgebras by means of the closed homomorphisms, the full homomorphisms and the homomorphisms, respectively. 
Definition 29. Let $\mathbf{A}$ be a partial $\Sigma$-algebra and $X \subseteq A$. Given $(\mathbf{s}, s) \in S^{\star} \times S$ and $\sigma \in \Sigma_{\mathbf{s}, s}$, we will say that $X$ is closed under the partial operation $F_{\sigma}: A_{\mathbf{s}} \longrightarrow A_{s}$ if, for every $\left(a_{j}\right)_{j \in|\mathbf{s}|} \in X_{\mathbf{s}}$, if $\left(a_{j}\right)_{j \in|\mathbf{s}|} \in \operatorname{Dom}\left(F_{\sigma}\right)$, then $F_{\sigma}\left(\left(a_{j}\right)_{j \in|\mathbf{s}|}\right) \in X_{s}$. We will say that $X$ is a closed subset of $\mathbf{A}$ if $X$ is closed under the partial operations of $\mathbf{A}$. We will denote by $\mathrm{Cl}(\mathbf{A})$ the set of all closed subsets of $\mathbf{A}$ (which is an algebraic closure system on $A)$ and by $\mathbf{C l}(\mathbf{A})$ the algebraic lattice $(\mathrm{Cl}(\mathbf{A}), \subseteq)$.

We will say that a partial $\Sigma$-algebra $\mathbf{B}=(B, G)$ is a subalgebra of $\mathbf{A}$ if $B$ is a closed subset of $\mathbf{A}$, for every $(\mathbf{s}, s) \in S^{\star} \times S$ and every $\sigma \in \Sigma_{\mathbf{s}, s}, \operatorname{Dom}\left(G_{\sigma}\right)=\operatorname{Dom}\left(F_{\sigma}\right) \cap B_{w}$ and, for every $b \in \operatorname{Dom}\left(G_{\sigma}\right), G_{\sigma}(b)=F_{\sigma}(b)$. We will denote by $\operatorname{Sub}(\mathbf{A})$ the set of all subalgebras of $\mathbf{A}$ and $b y$ $\operatorname{Sub}(\mathbf{A})$ the ordered set $(\operatorname{Sub}(\mathbf{A}), \subseteq)$. Since $\mathbf{C l}(\mathbf{A})$ and $\mathbf{S u b}(\mathbf{A})$ are isomorphic, we shall feel free to deal either with a closed subset of $\mathbf{A}$ or with the correlated subalgebra of $\mathbf{A}$, whichever is most convenient for the work at hand.

Remark 12. Let $\mathbf{A}$ and $\mathbf{B}$ be partial $\Sigma$-algebras such that $B \subseteq A$. Then $\mathbf{B}=(B, G)$ is a subalgebra of $\mathbf{A}$ if and only if, for every $(\mathbf{s}, s) \in S^{\star} \times S$ and every $\sigma \in \Sigma_{\mathbf{s}, s}, \Gamma_{G_{\sigma}}=\Gamma_{F_{\sigma}} \cap\left(B_{\mathbf{s}} \times A_{s}\right)$.

Remark 13. Let $\mathbf{A}$ be a partial $\Sigma$-algebra. If, for every $s \in S$ and every $\sigma \in \Sigma_{\lambda, s}, \operatorname{Dom}\left(F_{\sigma}\right)=\varnothing$, then $\varnothing^{S} \in \mathrm{Cl}(\mathbf{A})$. Let us note that, in such a case, $\varnothing^{S}$, the subalgebra of $\mathbf{A}$ canonically associated to $\varnothing^{S}$, is such that, for every $(\mathbf{s}, s) \in S^{\star} \times S$ and every $\sigma \in \Sigma_{\mathbf{s}, S}$, the partial operation from $\varnothing_{\mathbf{s}}^{S}$ to $\varnothing_{S}^{S}=\varnothing$ associated to $\sigma$ is discrete.

We next define the notion of closed homomorphism from a partial $\Sigma$-algebra to another which will allow us, among other things, to provide another characterization of the isomorphisms and of the subalgebras of a partial $\Sigma$-algebra.

Definition 30. Let $\mathbf{A}$ and $\mathbf{B}$ be partial $\Sigma$-algebras and $f$ a homomorphism from $\mathbf{A}$ to $\mathbf{B}$. We will say that $f$ is closed if, for every $(\mathbf{s}, s) \in S^{\star} \times S$, every $\sigma \in \Sigma_{\mathbf{s}, s}$ and every $\left(a_{j}\right)_{j \in|\mathbf{s}|} \in A_{\mathbf{s}}$, if $f_{\mathbf{s}}\left(\left(a_{j}\right)_{j \in|\mathbf{s}|}\right) \in \operatorname{Dom}\left(G_{\sigma}\right)$, then $\left(a_{j}\right)_{j \in|\mathbf{s}|} \in \operatorname{Dom}\left(F_{\sigma}\right)$, i.e., $\operatorname{Dom}\left(F_{\sigma}\right) \supseteq f_{\mathbf{s}}^{-1}\left[\operatorname{Dom}\left(G_{\sigma}\right)\right]$ (therefore $\operatorname{Dom}\left(F_{\sigma}\right)=f_{\mathbf{s}}^{-1}\left[\operatorname{Dom}\left(G_{\sigma}\right)\right]$ ).

Remark 14. A homomorphism is closed if and only if the domains of the partial operations in the source are exactly the inverse images of the domains of the corresponding operations in the target.

Remark 15. Every homomorphism between $\Sigma$-algebras is a closed homomorphism.

Proposition 18. Let $f$ be a homomorphism from A to B. Then the following statements are equivalent:

1. $f$ is an isomorphism from $\mathbf{A}$ to $\mathbf{B}$, i.e., $f$ is a section and a retraction.

2. $f$ is bijective and closed, i.e., for every $(\mathbf{s}, s) \in S^{\star} \times S$, every $\sigma \in \Sigma_{\mathbf{s}, s}$ and every $\left(a_{j}\right)_{j \in|\mathbf{s}|} \in$ $A_{\mathbf{s}}$, the following holds: $\left(a_{j}\right)_{j \in|\mathbf{s}|} \in \operatorname{Dom}\left(F_{\sigma}\right)$ if, and only if, $f_{\mathbf{s}}\left(\left(a_{j}\right)_{j \in|\mathbf{s}|}\right)=\left(f_{s_{j}}\left(a_{j}\right)\right)_{j \in|\mathbf{s}|} \in$ $\operatorname{Dom}\left(G_{\sigma}\right)$.

Proposition 19. Let $\mathbf{A}$ and $\mathbf{B}$ be partial $\Sigma$-algebras, $f$ a homomorphism from $\mathbf{A}$ to $\mathbf{B}$ and $X$ and $Y$ closed subsets of $\mathbf{A}$ and $\mathbf{B}$, respectively. Then:

1. $f^{-1}[Y]$ is a closed subset of $\mathbf{A}$.

2. If $f$ is closed, then $f[X]$ is a closed subset of $\mathbf{B}$.

Remark 16. For a homomorphism $f$ from a partial $\Sigma$-algebra $\mathbf{A}$ to another $\mathbf{B}$ and a subset $X$ of $A$, we have that $f\left[\operatorname{Sg}_{\mathbf{A}}(X)\right] \subseteq \operatorname{Sg}_{\mathbf{B}}(f[X])$ (the converse inclusion does not holds in general; however, if $f$ is closed, then $f\left[\operatorname{Sg}_{\mathbf{A}}(X)\right]=\operatorname{Sg}_{\mathbf{B}}(f[X])$.

Proposition 20. Let $\mathbf{A}$ and $\mathbf{B}$ be partial $\sum$-algebras such that $B \subseteq A$. Then $\mathbf{B}$ is a subalgebra of $\mathbf{A}$ if and only if in $^{\mathbf{B}, \mathbf{A}}=\left(\mathbf{B}, \mathrm{in}^{B, A}, \mathbf{A}\right)$ is a closed homomorphism from $\mathbf{B}$ to $\mathbf{A}$. 
Definition 31. Let $\mathbf{A}$ be a partial $\Sigma$-algebra. Then we will denote by $\mathrm{Sg}_{\mathrm{A}}$ the algebraic closure operator canonically associated to the algebraic closure system $\mathrm{Cl}(\mathbf{A})$ on $A$ and we call it the subalgebra generating operator for $\mathbf{A}$. Moreover, if $X \subseteq A$, then we call $\mathrm{Sg}_{\mathbf{A}}(X)$ the subalgebra of $\mathbf{A}$ generated by $X$, and if $X$ is such that $\operatorname{Sg}_{\mathbf{A}}(X)=A$, then we will say that $X$ is a generating subset of $\mathbf{A}$. Besides, $\mathbf{S g}_{\mathbf{A}}(X)$ denotes the partial $\Sigma$-algebra determined by $\operatorname{Sg}_{\mathbf{A}}(X)$.

Remark 17. Let $\mathbf{A}$ be a partial E-algebra. Then the algebraic closure operator $\mathrm{Sg}_{\mathrm{A}}$ is uniform, i.e., for every $X, Y \subseteq A$, if $\operatorname{supp}_{S}(X)=\operatorname{supp}_{S}(Y)$, then we have $\operatorname{supp}_{S}\left(\operatorname{Sg}_{\mathbf{A}}(X)\right)=$ $\operatorname{supp}_{S}\left(\operatorname{Sg}_{\mathbf{A}}(Y)\right)$.

Proposition 21 (Principle of Proof by Algebraic Induction). Let A be a partial $\Sigma$-algebra generated by $X$. Then to prove that a subset $Y$ of $A$ is equal to $A$ it suffices to show:

1. $X \subseteq Y$ (algebraic induction basis); and

2. $Y$ is a closed subset subalgebra of $\mathbf{A}$ (algebraic induction step).

We next state the principle of extension of identities. This principle, which is fundamental to elucidate the equality of two coterminal homomorphisms, will be used on several occasions in this work.

Proposition 22. Let $f, g: \mathbf{A} \longrightarrow \mathbf{B}$ be homomorphisms between partial $\Sigma$-algebras and let $X$ be $a$ subset of $\mathbf{A}$. If $f\rceil_{X}=g \uparrow_{X}$, then $f \uparrow_{\mathbf{S g}_{\mathbf{A}}(X)}=g \uparrow_{\mathbf{S g}_{\mathbf{A}}}(X)$. In particular, if $X$ is a generating subset of $\mathbf{A}$ and $f\rceil X_{X}=g \uparrow_{X}$, then $f=g$

Definition 32. Let $\mathbf{A}$ be a partial $\Sigma$-algebra. We will say that a partial $\Sigma$-algebra $\mathbf{B}=(B, G)$ is a relative subalgebra of $\mathbf{A}$ if $B \subseteq A$ and, for every $(\mathbf{s}, s) \in S^{\star} \times S$ and every $\sigma \in \Sigma_{\mathbf{s}, s}$, $\operatorname{Dom}\left(G_{\sigma}\right) \subseteq \operatorname{Dom}\left(F_{\sigma}\right)$ and, for every $\left(b_{j}\right)_{j \in|\mathbf{s}|} \in B_{\mathbf{s}}$, if $\left(b_{j}\right)_{j \in|\mathbf{s}|} \in \operatorname{Dom}\left(F_{\sigma}\right)$ and $F_{\sigma}\left(\left(b_{j}\right)_{j \in|\mathbf{s}|}\right) \in$ $B_{s}$, then $\left(b_{j}\right)_{j \in|\mathbf{s}|} \in \operatorname{Dom}\left(G_{\sigma}\right)$ and $G_{\sigma}\left(\left(b_{j}\right)_{j \in|\mathbf{s}|}\right)=F_{\sigma}\left(\left(b_{j}\right)_{j \in|\mathbf{s}|}\right)$. We will denote by $\operatorname{Sub}_{\mathbf{r}}(\mathbf{A})$ the set of all relative subalgebras of $\mathbf{A}$.

Remark 18. Let $\mathbf{A}$ and $\mathbf{B}$ be partial $\Sigma$-algebras such that $B \subseteq$ A. Then $\mathbf{B}=(B, G)$ is a relative subalgebra of $\mathbf{A}$ if and only if, for every $(\mathbf{s}, s) \in S^{\star} \times S$ and every $\sigma \in \Sigma_{\mathbf{s}, s}, \Gamma_{G_{\sigma}}=\Gamma_{F_{\sigma}} \cap\left(B_{\mathbf{s}} \times B_{s}\right)$.

Remark 19. Let $\mathbf{A}=(A, F)$ be a partial $\Sigma$-algebra and $X \subseteq A$. Then there exists a unique structure of partial $\Sigma$-algebra $\mathrm{L}^{X, A}(F)$ on $X$, the optimal lift of $F$ with respect to in $X, A$, such that, for every partial $\sum$-algebra $C$ and every mapping $h$ from $C$ to $X$, if in $X, A \circ h$ is a homomorphism from $\mathbf{C}$ to $\mathbf{A}$, then $h$ is a homomorphism from $\mathbf{C}$ to $\left(X, \mathrm{~L}^{\mathrm{in}}{ }^{X}(F)\right)$. In fact, it suffices to take, for every $(\mathbf{s}, s) \in S^{\star} \times S$ and every $\sigma \in \Sigma_{\mathbf{s}, s}$, as partial operation associated to $\sigma$ the partial mapping $\mathrm{L}^{\mathrm{in}^{X, A}}(F)_{\sigma}$ from $X_{\mathbf{s}}$ to $X_{S}$ defined as follows:

$$
\operatorname{Dom}\left(\mathrm{L}^{\mathrm{in}^{X, A}}(F)_{\sigma}\right)=\left\{x \in X_{\mathbf{s}} \mid x \in \operatorname{Dom}\left(F_{\sigma}\right) \& F_{\sigma}(x) \in X_{s}\right\}
$$

and, for every $x \in \operatorname{Dom}\left(\mathrm{L}^{\mathrm{in}^{X, A}}(F)_{\sigma}\right), \mathrm{L}^{\mathrm{in}^{X, A}}(F)_{\sigma}(x)=F_{\sigma}(x)$. This shows that every subset $X$ of $A$ is the underlying $S$-sorted set of exactly one relative subalgebra of $\mathbf{A}$, and that the structure of partial $\Sigma$-algebra on $X$ is uniquely determined by the structure of partial $\Sigma$-algebra on $A$ and the specification of the subset $X$ of $A$.

We next define the notion of full homomorphism from a partial $\Sigma$-algebra to another which will allow us, among other things, to provide another characterization of the isomorphisms and of the relative subalgebras of a $\Sigma$-algebra.

Definition 33. Let $\mathbf{A}$ and $\mathbf{B}$ be partial $\Sigma$-algebras and $f$ a homomorphism from $\mathbf{A}$ to $\mathbf{B}$. We will say that $f$ is full if, for every $(\mathbf{s}, s) \in S^{\star} \times S$, every $\sigma \in \Sigma_{\mathbf{s}, s}$ and every $\left(a_{j}\right)_{j \in|\mathbf{s}|} \in A_{\mathbf{s}}$, if $f_{\mathbf{s}}\left(\left(a_{j}\right)_{j \in|\mathbf{s}|}\right) \in \operatorname{Dom}\left(G_{\sigma}\right)$ and $G_{\sigma}\left(f_{\mathbf{s}}\left(\left(a_{j}\right)_{j \in|\mathbf{s}|}\right)\right) \in f_{s}\left[A_{s}\right]$, then there exists an $\left(a_{j}^{\prime}\right)_{j \in|\mathbf{s}|} \in$ $\operatorname{Dom}\left(F_{\sigma}\right)$ such that $f_{\mathbf{s}}\left(\left(a_{j}^{\prime}\right)_{j \in|\mathbf{s}|}\right)=f_{\mathbf{s}}\left(\left(a_{j}\right)_{j \in|\mathbf{s}|}\right)$. 
Remark 20. Those homomorphisms that are full and injective are characterizable as those that are initial and injective, where a homomorphism $f$ from a partial $\Sigma$-algebra $\mathbf{A}$ to another $\mathbf{B}$ is said to be initial if, for every partial $\Sigma$-algebra $\mathbf{C}$ and every $S$-sorted mapping $h$ from $C$ to $A$, if $f \circ h$ is $a$ homomorphism from $\mathbf{C}$ to $\mathbf{B}$, then $h$ is a homomorphism from $\mathbf{C}$ to $\mathbf{A}$.

Proposition 23. Let $f$ be a homomorphism from A to B. Then the following statements are equivalent:

1. $f$ is an isomorphism from $\mathbf{A}$ to $\mathbf{B}$, i.e., $f$ is a section and a retraction.

2. $f$ is bijective and full.

Proposition 24. Let $\mathbf{A}$ and $\mathbf{B}$ be partial $\Sigma$-algebras such that $B \subseteq A$. Then $\mathbf{B}$ is a relative subalgebra of $\mathbf{A}$ if and only if in ${ }^{\mathbf{B}, \mathbf{A}}=\left(\mathbf{B}, \mathrm{in}^{B, A}, \mathbf{A}\right)$ is a full homomorphism from $\mathbf{B}$ to $\mathbf{A}$.

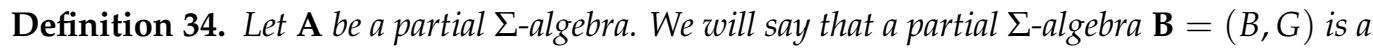
weak subalgebra of $\mathbf{A}$ if $B \subseteq A$ and, for every $(\mathbf{s}, s) \in S^{\star} \times S$, every $\sigma \in \Sigma_{\mathbf{s}, s}$ and every $b \in B_{\mathbf{s}}$, if $b \in \operatorname{Dom}\left(G_{\sigma}\right)$, then $b \in \operatorname{Dom}\left(F_{\sigma}\right)$ and $G_{\sigma}(b)=F_{\sigma}(b)$. We will denote by $\operatorname{Sub}_{\mathrm{W}}(\mathbf{A})$ the set of all weak subalgebras of $\mathbf{A}$.

Remark 21. Let $\mathbf{A}$ and $\mathbf{B}$ be partial $\Sigma$-algebras such that $B \subseteq A$. Then $\mathbf{B}=(B, G)$ is a weak subalgebra of $\mathbf{A}$ if and only if, for every $(\mathbf{s}, s) \in S^{\star} \times S$ and every $\sigma \in \Sigma_{\mathbf{s}, s}, \Gamma_{G_{\sigma}} \subseteq \Gamma_{F_{\sigma}}$.

Proposition 25. Let $\mathbf{A}$ and $\mathbf{B}$ be partial $\Sigma$-algebras such that $B \subseteq A$. Then $\mathbf{B}$ is a weak subalgebra of $\mathbf{A}$ if and only if in $^{\mathbf{B}, \mathbf{A}}=\left(\mathbf{B}, \mathrm{in}^{B, A}, \mathbf{A}\right)$ is a homomorphism from $\mathbf{B}$ to $\mathbf{A}$.

Remark 22. Since every closed homomorphism is a full homomorphism and every full homomorphism is a homomorphism, it follows that every subalgebra is a relative subalgebra and every relative subalgebra is a weak subalgebra. Moreover, every relative subalgebra on a closed subset is a subalgebra.

Proposition 26. Let $\mathbf{A}$ and $\mathbf{B}$ be partial $\Sigma$-algebras and $f: A \longrightarrow B$ a mapping from $A$ to $B$. Then:

1. If $f: \mathbf{A} \longrightarrow \mathbf{B}$ is a homomorphism, then $\Gamma_{f}$ is a closed subset of $\mathbf{A} \times \mathbf{B}$.

2. If $\mathbf{B}$ is a $\Sigma$-algebra and $\Gamma_{f}$ is a closed subset of $\mathbf{A} \times \mathbf{B}$, then $f$ is a homomorphism from $\mathbf{A}$ to $\mathbf{B}$.

Remark 23. Notice the (partial) analogy of the just stated proposition and the closed graph theorem in point-set topology.

Lemma 1. Let $f: \mathbf{A} \longrightarrow \mathbf{B}$ be a homomorphism, $X \subseteq A, g: X \longrightarrow B$ such that $\Gamma_{g} \subseteq \Gamma_{f}$ and $\mathbf{X}$ the relative subalgebra of $\mathbf{A}$ on $X$. Then:

1. $g: \mathbf{X} \longrightarrow \mathbf{B}$ is a homomorphism.

2. $\Gamma_{g}$ is a closed subset of $\mathbf{A} \times \mathbf{B}$ if and only if $X$ is a closed subset of $\mathbf{A}$.

Corollary 2. Let $f: \mathbf{A} \longrightarrow \mathbf{B}$ be a homomorphism and $X \subseteq$ A. Then the following statements are equivalent:

1. $\operatorname{Sg}_{\mathbf{A}}(X)=A$.

2. $\operatorname{Sg}_{\mathbf{A} \times \mathbf{B}}\left(\Gamma_{f\lceil X}\right)=\Gamma_{f}$.

The following technical lemma will become quite useful later on.

Lemma 2. Let $\mathbf{A}, \mathbf{B}, \mathbf{C}$ and $\mathbf{D}$ be partial $\Sigma$-algebras, $\mathbf{C}$ a weak subalgebra of $\mathbf{A}$ such that $\mathrm{Sg}_{\mathbf{A}}(C)=$ $A, \mathbf{D}$ a relative subalgebra of $\mathbf{B}$ and $f$ a homomorphism from $\mathbf{C}$ to $\mathbf{D}$ which allows a homomorphic extension $\bar{f}$ from $\mathbf{A}$ to $\mathbf{B}$. Then: 
1. $\operatorname{Sg}_{\mathbf{A} \times \mathbf{D}}\left(\Gamma_{f}\right)$ is the underlying function of a homomorphism $f^{b}$ from $\operatorname{Dom}\left(\operatorname{Sg}_{\mathbf{A} \times \mathbf{D}}\left(\Gamma_{f}\right)\right)$ to $\mathbf{D}$ (where $\operatorname{Dom}\left(\operatorname{Sg}_{\mathbf{A} \times \mathbf{D}}\left(\Gamma_{f}\right)\right)$ is the relative subalgebra of $\mathbf{A}$ whose underlying many-sorted set is $\left.\operatorname{Dom}\left(\operatorname{Sg}_{\mathbf{A} \times \mathbf{D}}\left(\Gamma_{f}\right)\right)\right)$ and $\operatorname{Dom}\left(\operatorname{Sg}_{\mathbf{A} \times \mathbf{D}}\left(\Gamma_{f}\right)\right)$ is generated by $C$.

2. If $\mathbf{E}$ is a $\mathbf{C}$-generated relative subalgebra of $\mathbf{A}$ and $g: \mathbf{E} \longrightarrow \mathbf{D}$ is a homomorphic extension of $f$, then $\Gamma_{g} \subseteq \Gamma_{f}$.

3. If $\mathbf{A}$ is a $\sum$-algebra, then $f^{b}$ is a closed homomorphism from $\operatorname{Dom}\left(\operatorname{Sg}_{\mathbf{A} \times \mathbf{D}}\left(\Gamma_{f}\right)\right)$ to $\mathbf{D}$; and if $f^{b}$ is closed and $g: \mathbf{E} \longrightarrow \mathbf{D}$ is any closed homomorphic extension of $f$ such that $\mathbf{E}$ is a $C$-generated relative subalgebra of $\mathbf{A}$, then $g=f^{b}$.

Definition 35. Let $\mathbf{A}$ and $\mathbf{B}$ be partial $\Sigma$-algebras and $f$ a homomorphism from $\mathbf{A}$ to $\mathbf{B}$. We will say that $f$ is dense if $f[A]$ generates $\mathbf{B}$, i.e., if $\operatorname{Sg}_{\mathbf{B}}(f[A])=B$.

Lemma 3. Let $\mathbf{A}$ and $\mathbf{B}$ be partial $\Sigma$-algebras and $f$ a homomorphism from $\mathbf{A}$ to $\mathbf{B}$. Then $f$ is an epimorphism if and only if $f$ is dense.

Remark 24. If a homomorphism between partial $\Sigma$-algebras is dense and closed, then it is surjective (because the image of a closed homomorphism is a closed subset of the target).

Proposition 27. Let $\mathbf{A}$ and $\mathbf{B}$ be partial $\Sigma$-algebras and $f$ a homomorphism from $\mathbf{A}$ to $\mathbf{B}$. Then there exists a unique epimorphism $f$ from $\mathbf{A}$ to $\mathbf{S g}_{\mathbf{B}}(f[A])$ such that $f=$ in $\mathbf{S g}_{\mathbf{B}}[f[A]] \circ f^{\mathrm{e}}$, where $\operatorname{in}^{\mathbf{S g}_{\mathbf{B}}(f[A])}$ is the closed embedding of $\mathbf{S g}_{\mathbf{B}}(f[A])$ into $\mathbf{B}$. Moreover, (1) $f$ is an isomorphism if and only if $f$ is closed and injective; and (2) for every partial $\Sigma$-algebra $\mathbf{C}$, every epimorphism $g$ from $\mathbf{A}$ to $\mathbf{C}$ and every closed injective homomorphism $h$ from $\mathbf{C}$ to $\mathbf{B}$, if $f=h \circ g$, then there exists a unique isomorphism $k$ from $\mathbf{S g}_{\mathbf{B}}(f[A])$ to $\mathbf{C}$ such that $g=k \circ f$ and $h \circ k=$ in $\mathbf{S g}_{\mathbf{B}}(f[A])$.

Therefore (Epimorphisms, Closed and injective homomorphisms) is a factorization system in PAlg $(\Sigma)$.

Congruences provide an internal description of the homomorphic images from a partial $\Sigma$-algebra and closed congruences provide an internal description of the closed homomorphic images from a partial $\Sigma$-algebra. Moreover, closed congruences are fundamental for the description of the varieties of partial $\Sigma$-algebras defined by existentially conditional existence equations.

Definition 36. Let $\mathbf{A}$ be a partial $\Sigma$-algebra and $\Phi$ an S-sorted equivalence on $A$. We will say that $\Phi$ is an $S$-sorted congruence on (or, to abbreviate, a congruence on) $\mathbf{A}$ if, for every $(\mathbf{s}, s) \in\left(S^{\star}-\right.$ $\{\lambda\}) \times S$, every $\sigma \in \Sigma_{\mathbf{s}, s}$, and every $\left(a_{j}\right)_{j \in|\mathbf{s}|},\left(b_{j}\right)_{j \in|\mathbf{s}|} \in A_{\mathbf{s}}$, if, for every $j \in|\mathbf{s}|,\left(a_{j}, b_{j}\right) \in \Phi_{s_{j}}$, $\left(a_{j}\right)_{j \in|\mathbf{s}|} \in \operatorname{Dom}\left(F_{\sigma}\right)$ and $\left(b_{j}\right)_{j \in|\mathbf{s}|} \in \operatorname{Dom}\left(F_{\sigma}\right)$, then $\left(F_{\sigma}\left(\left(a_{j}\right)_{j \in|\mathbf{s}|}\right), F_{\sigma}\left(\left(b_{j}\right)_{j \in|\mathbf{s}|}\right)\right) \in \Phi_{s}$. We will denote by $\operatorname{Cgr}(\mathbf{A})$ the set of all $S$-sorted congruences on $\mathbf{A}$, which is an algebraic closure system on $A \times A$, by $\mathrm{Cg}_{\mathbf{A}}$ the corresponding algebraic closure operator, by $\mathbf{C g r}(\mathbf{A})$ the algebraic lattice $(\operatorname{Cgr}(\mathbf{A}), \subseteq)$, by $\nabla_{\mathbf{A}}$ the greatest element of $\mathbf{C g r}(\mathbf{A})$ and by $\Delta_{\mathbf{A}}$ the least element of $\mathbf{C g r}(\mathbf{A})$.

For a congruence $\Phi$ on $\mathbf{A}$, the quotient partial $\Sigma$-algebra of $\mathbf{A}$ by $\Phi$, denoted by $\mathbf{A} / \Phi$, is the partial $\Sigma$-algebra $\left(A / \Phi, F^{\mathbf{A} / \Phi}\right)$, where, for every $(\mathbf{s}, s) \in S^{\star} \times S$ and every $\sigma \in \Sigma_{\mathbf{s}, s}$, the domain of the partial operation $F_{\sigma}^{\mathbf{A} / \Phi}$, also denoted, to simplify, by $F_{\sigma}$, from $(A / \Phi)_{\mathbf{s}}$ to $A_{s} / \Phi_{s}$ is the set

$$
\left\{\left(\left[a_{j}\right]_{\Phi_{s_{j}}}\right)_{j \in|\mathbf{s}|} \in(A / \Phi)_{\mathbf{s}}\left|\exists\left(a_{j}^{\prime}\right)_{j \in|\mathbf{s}|} \in A_{\mathbf{s}} \forall j \in\right| \mathbf{s} \mid\left(\left(a_{j}, a_{j}^{\prime}\right) \in \Phi_{s_{j}}\right)\right\},
$$

and if $\left(\left[a_{j}\right]_{\Phi_{s_{j}}}\right)_{j \in|\mathbf{s}|}$ in $\operatorname{Dom}\left(F_{\sigma}\right)$, then

$$
F_{\sigma}\left(\left(\left[a_{j}\right]_{\Phi_{s_{j}}}\right)_{j \in|\mathbf{s}|}\right)=\left[F_{\sigma}\left(\left(a_{j}\right)_{j \in|\mathbf{s}|}\right)\right]_{\Phi_{s}}
$$

and the canonical projection from $\mathbf{A}$ to $\mathbf{A} / \Phi$, denoted by $\mathrm{pr}^{\Phi}: \mathbf{A} \longrightarrow \mathbf{A} / \Phi$, is the full and surjective homomorphism determined by the projection from $A$ to $A / \Phi$. The ordered pair $\left(\mathbf{A} / \Phi, \operatorname{pr}^{\Phi}\right)$ has the following universal property: $\operatorname{Ker}\left(\mathrm{pr}^{\Phi}\right)$ is $\Phi$ and, for every $\Sigma$-algebra $\mathbf{B}$ and every homomorphism $f$ from $\mathbf{A}$ to $\mathbf{B}$, if $\operatorname{Ker}(f) \supseteq \Phi$, then there exists a unique homomorphism $h$ from $\mathbf{A} / \Phi$ 
to $\mathbf{B}$ such that $h \circ \mathrm{pr}^{\Phi}=f$. In particular, if $\Psi$ is a congruence on $A$ such that $\Phi \subseteq \Psi$, then we will denote by $\mathrm{p}^{\Phi, \Psi}$ the unique homomorphism from $\mathbf{A} / \Phi$ to $\mathbf{A} / \Psi$ such that $\mathrm{p}^{\Phi, \Psi} \circ \mathrm{pr}^{\Phi}=\mathrm{pr}^{\Psi}$.

We will say that $\Phi$ is an $S$-sorted closed congruence on (or, to abbreviate, a closed congruence on) $\mathbf{A}$ if $\Phi$ is a congruence on $\mathbf{A}$ and, for every $(\mathbf{s}, s) \in\left(S^{\star}-\{\lambda\}\right) \times S$, every $\sigma \in \Sigma_{\mathbf{s}, s}$, and every $\left(a_{j}\right)_{j \in|\mathbf{s}|,}\left(b_{j}\right)_{j \in|\mathbf{s}|} \in A_{\mathbf{s}}$, if, for every $j \in|\mathbf{s}|,\left(a_{j}, b_{j}\right) \in \Phi_{s_{j}}$ and $\left(a_{j}\right)_{j \in|\mathbf{s}|} \in$ $\operatorname{Dom}\left(F_{\sigma}\right)$, then $\left(b_{j}\right)_{j \in|\mathbf{s}|} \in \operatorname{Dom}\left(F_{\sigma}\right)$. We will denote by $\operatorname{Cgr}_{\mathbf{c}}(\mathbf{A})$ the set of all $S$-sorted closed congruences on $\mathbf{A}$. The congruence $\Delta_{\mathbf{A}}$ is a closed congruence on $\mathbf{A}$, while $\nabla_{\mathbf{A}}$ is closed if and only if, for every $(\mathbf{s}, s) \in\left(S^{\star} \times S\right.$ and every $\sigma \in \Sigma_{\mathbf{s}, s}, F_{\sigma}$ is either total or discrete. If $\Phi$ and $\Psi$ are closed congruences on $\mathbf{A}$, then $\mathrm{Cg}_{\mathbf{A}}(\Phi \cup \Psi)$, their supremum in $\mathbf{C g r}(\mathbf{A})$, is a closed congruence on $\mathbf{A}$ and it is the least equivalence relation containing them, i.e., $\mathrm{Cg}_{\mathbf{A}}(\Phi \cup \Psi)=\bigcup_{n \in \mathbb{N}}(\Psi \circ \Phi)^{n}$ (let us point out that, in general, the supremum of two non-closed congruences is not necessarily the least equivalence relation containing them). The set $\operatorname{Cgr}_{c}(\mathbf{A})$ is a principal ideal of $\mathbf{C g r}(\mathbf{A})$ precisely the one determined by $\bigcup_{\Phi \in \operatorname{Cgr}_{\mathrm{c}}(\mathbf{A})} \Phi$, which is the largest closed congruence on $\mathbf{A}$.

Remark 25. Let A be a partial L-algebra and $\Phi$ an S-sorted equivalence on $A$. Then $\Phi$ is a congruence on $\mathbf{A}$ if and only if $\Phi$ is a subalgebra of $\mathbf{A} \times \mathbf{A}$.

Remark 26. Let $\mathbf{A}$ be a partial $\Sigma$-algebra and $\Phi$ an $S$-sorted congruence on $A$, then the full and surjective homomorphism $\mathrm{pr}^{\Phi}$ from $\mathbf{A}$ to $\mathbf{A} / \Phi$ is final and surjective, where a homomorphism $f$ from a partial $\Sigma$-algebra $\mathbf{A}$ to another $\mathbf{B}$ is said to be final if, for every partial $\Sigma$-algebra $\mathbf{C}$ and every $S$-sorted mapping $h$ from $B$ to $C$, if $h \circ f$ is a homomorphism from $\mathbf{A}$ to $\mathbf{C}$, then $h$ is a homomorphism from $\mathbf{B}$ to $\mathbf{C}$. For a homomorphism $f$ from a partial $\sum$-algebra $\mathbf{A}$ to another $\mathbf{B}$ the property of being final is very interesting because it allows one to state that an S-sorted mapping $h$ from $B$ to $C$, the underlying set of another partial $\Sigma$-algebra $\mathbf{C}$, is a homomorphism if its composition with the subjacent application of the homomorphism $f$ is a homomorphism from $\mathbf{A}$ to $\mathbf{C}$. Let us point out that a homomorphism $f$ from a partial $\Sigma$-algebra $\mathbf{A}=(A, F)$ to another $\mathbf{B}=(B, G)$ is final if and only if, for every $(\mathbf{s}, s) \in S^{\star} \times S$ and every $\sigma \in \Sigma_{\mathbf{s}, s}, \Gamma_{G_{\sigma}}=\left(f_{\mathbf{s}} \times f_{s}\right)\left[\Gamma_{F_{\sigma}}\right]$.

Remark 27. Let A be a partial E-algebra. If $\Phi$ an S-sorted closed congruence on $A$, then $\mathrm{pr}^{\Phi}: \mathbf{A} \longrightarrow \mathbf{A} / \Phi$, is a closed and surjective homomorphism.

Remark 28. Let ClfdPAlg $(\Sigma)$ be the category whose objects are the classified partial $\Sigma$-algebras, i.e, the ordered pairs $(\mathbf{A}, \Phi)$ where $\mathbf{A}$ is a partial $\Sigma$-algebra and $\Phi$ a congruence on $\mathbf{A}$, and in which the set of morphisms from $(\mathbf{A}, \Phi)$ to $(\mathbf{B}, \Psi)$ is the set of all homomorphisms $f$ from $\mathbf{A}$ to $\mathbf{B}$ such that, for every $s \in S$ and every $(x, y) \in A_{s}^{2}$, if $(x, y) \in \Phi_{s}$, then $\left(f_{\mathcal{S}}(x), f_{\mathcal{S}}(y)\right) \in \Psi_{\mathcal{s}}$. Let $G$ be the functor from $\mathbf{P A} \lg (\Sigma)$ to $\mathbf{C l f d P A} \lg (\Sigma)$ whose object mapping sends $\mathbf{A}$ to $\left(\mathbf{A}, \Delta_{\mathbf{A}}\right)$ and whose morphism mapping sends $f: \mathbf{A} \longrightarrow \mathbf{B}$ to $f:\left(\mathbf{A}, \Delta_{\mathbf{A}}\right) \longrightarrow\left(\mathbf{B}, \Delta_{\mathbf{B}}\right)$. Then, for every classified partial $\Sigma$-algebra $(\mathbf{A}, \Phi)$, there exists a universal mapping from $(\mathbf{A}, \Phi)$ to $G$, which is precisely the ordered pair $\left(\mathbf{A} / \Phi, \operatorname{pr}^{\Phi}\right)$ with $\operatorname{pr}^{\Phi}:(\mathbf{A}, \Phi) \longrightarrow\left(\mathbf{A} / \Phi, \Delta_{\mathbf{A} / \Phi}\right)$.

Remark 29. Let $\mathbf{A}=(A, F)$ be a partial $\Sigma$-algebra and $\Phi$ an $S$-sorted equivalence on $A$. Then there exists a unique structure of partial $\Sigma$-algebra $\mathrm{L}_{\mathrm{pr}^{\Phi}}(F)$ on $A / \Phi$, the co-optimal lift of $F$ with respect to $\mathrm{pr}^{\Phi}$, such that, for every partial $\Sigma$-algebra $\mathbf{C}$ and every mapping $h$ from $A / \Phi$ to $C$, if $h \circ \mathrm{pr}^{\Phi}$ is a homomorphism from $\mathbf{A}$ to, $\mathbf{C}$ then $h$ is a homomorphism from $\mathbf{C}$ to $\left(A / \Phi, \mathrm{L}_{\mathrm{pr}^{\Phi}}(F)\right)$ if and only if $\Phi$ is an $S$-sorted congruence on $\mathbf{A}$. This shows that, for every $S$-sorted congruence $\Phi$ on $\mathbf{A} A, A / \Phi$ is the underlying $S$-sorted set of exactly one $\Sigma$-algebra, and that the structure of partial $\Sigma$-algebra on $A / \Phi$ is uniquely determined by the structure of partial $\Sigma$-algebra on $A$ and the specification of the $S$-sorted congruence $\Phi$ on $A$. What we have just said is, in fact, a particular case of the following: for a partial $\Sigma$-algebra $\mathbf{A}=(A, F)$ and an $S$-sorted mapping $f$ from $A$ to $B$, there exists a co-optimal lift of $F$ with respect to $f$ if and only if $\operatorname{Ker}(f)$ is a congruence on $\mathbf{A}$.

We next state the weak homomorphism theorem.

Proposition 28. Let A, B and C be partial $\Sigma$-algebras, $f$ a full and surjective homomorphism from $\mathbf{A}$ to $\mathbf{B}$ and $g$ a homomorphism from $\mathbf{A}$ to $\mathbf{C}$. If $\operatorname{Ker}(g) \supseteq \operatorname{Ker}(f)$, then there exists a unique 
homomorphism $h$ from $\mathbf{B}$ to $\mathbf{C}$ such that $g=h \circ f$ (obviously, if there exists a homomorphism $h$ from $\mathbf{B}$ to $\mathbf{C}$ such that $g=h \circ f$, then $\operatorname{Ker}(g) \supseteq \operatorname{Ker}(f)$ ). Moreover,

1. $h$ is a full homomorphism if and only if $g$ is a full homomorphism;

2. $h$ is an isomorphism if and only if $g$ is a surjective and full homomorphism and $\operatorname{Ker}(g)=$ $\operatorname{Ker}(f)$;

3. if $g$ is closed, then $h$ is closed;

4. if $f$ is closed, then $h$ is closed if and only if $g$ is closed.

From the just stated proposition we obtain the weak isomorphism theorem.

Corollary 3. Let $\mathbf{A}$ and $\mathbf{B}$ be partial $\Sigma$-algebras and $f$ a full and surjective homomorphism from $\mathbf{A}$ to $\mathbf{B}$. Then $\mathbf{B}$ and $\mathbf{A} / \operatorname{Ker}(f)$ are isomorphic.

Proposition 29. Let $\mathbf{A}$ and $\mathbf{B}$ be partial $\Sigma$-algebras and $f$ a homomorphism from $\mathbf{A}$ to $\mathbf{B}$. Then there exists a unique injective homomorphism $f^{\mathrm{m}}$ from $\mathbf{A} / \operatorname{Ker}(f)$ to $\mathbf{B}$ such that $f=f^{\mathrm{m}} \circ \operatorname{pr}^{\operatorname{Ker}(f)}$. Moreover,

1. $f^{\mathrm{m}}$ is an isomorphism if and only if $f$ is full and surjective; and

2. for every partial $\Sigma$-algebra $\mathbf{C}$, every full and surjective homomorphism $g$ from $\mathbf{A}$ to $\mathbf{C}$ and every injective homomorphism $h$ from $\mathbf{C}$ to $\mathbf{B}$, if $f=h \circ g$, then there exists a unique isomorphism $k$ from $\mathbf{C}$ to $\mathbf{A} / \operatorname{Ker}(f)$ such that $k \circ g=\operatorname{pr}^{\operatorname{Ker}(f)}$ and $f^{\mathrm{m}} \circ k=h$.

Therefore (Full and surjective homomorphisms, Monomorphisms) is a factorization system in PAlg $(\Sigma)$.

\section{Partial Dedekind-Peano Algebras}

We next define for many-sorted partial algebras the counterpart of the notion of Dedekind-Peano algebra. Moreover, we state and prove the principle of definition by algebraic recursion for free many-sorted algebras with respect to many-sorted partial algebras and define a functor from a wide subcategory of $\mathbf{P A} \lg (\Sigma)$ to $\operatorname{Alg}(\Sigma)$ which will be used afterwards to obtain, from a subfunctor of that functor, an example of completion.

Definition 37. Let $\mathbf{A}$ be a partial L-algebra. We will say that $\mathbf{A}$ is a partial Dedekind-Peano $\Sigma$-algebra, abbreviated to PDP-algebra when this is unlikely to cause confusion, if the following axioms hold

PDP1For every $(\mathbf{s}, s) \in S^{\star} \times S$ and every $\sigma \in \Sigma_{\mathbf{s}, s}, F_{\sigma}: A_{\mathbf{s}} \longrightarrow A_{s}$ is injective, i.e., for every $\left(a_{j}\right)_{j \in|\mathbf{s}|},\left(b_{j}\right)_{j \in|\mathbf{s}|} \in \operatorname{Dom}\left(F_{\sigma}\right)$, if $F_{\sigma}\left(\left(a_{j}\right)_{j \in|\mathbf{s}|}\right)=F_{\sigma}\left(\left(b_{j}\right)_{j \in|\mathbf{s}|}\right)$, then $\left(a_{j}\right)_{j \in|\mathbf{s}|}=\left(b_{j}\right)_{j \in|\mathbf{s}|}$.

PDP2For every $s \in S$ and every $\sigma, \tau \in \Sigma$.,s, if $\sigma \neq \tau$, then $\operatorname{Im}\left(F_{\sigma}\right) \cap \operatorname{Im}\left(F_{\tau}\right)=\varnothing$, i.e., $F_{\sigma}\left[\operatorname{Dom}\left(F_{\sigma}\right)\right] \cap F_{\tau}\left[\operatorname{Dom}\left(F_{\tau}\right)\right]=\varnothing$.

$\operatorname{PDPBSg}_{\mathbf{A}}\left(A-\left(\cup_{\sigma \in \Sigma, s} \operatorname{Im}\left(F_{\sigma}\right)\right)_{s \in S}\right)=A$.

We call the $S$-sorted set $A-\left(\bigcup_{\sigma \in \Sigma, s} \operatorname{Im}\left(F_{\sigma}\right)\right)_{s \in S}$ the basis of Dedekind-Peano of $\mathbf{A}$, and we denote it by $\mathrm{B}(\mathbf{A})$.

Remark 30. A discrete partial $\sum$-algebra is always a PDP-algebra on its underlying S-sorted set. Moreover, every weak subalgebra of a PDP-algebra is a PDP-algebra.

We next state the principle of the definition by algebraic recursion for many-sorted PDP-algebras with respect to many-sorted algebras.

Proposition 30. Let $\mathbf{A}$ be a PDP-algebra, $\mathrm{B}(\mathbf{A})$ its basis, $\mathbf{B}$ a E-algebra and $f$ a mapping from $\mathrm{B}(\mathbf{A})$ to $B$. Then there exists a unique homomorphism $f^{\sharp}$ from $\mathbf{A}$ to $\mathbf{B}$ such that $f^{\sharp} \circ \mathrm{in}^{\mathrm{B}(\mathbf{A}), \mathbf{A}}=f$.

Proof. It suffices to take as $f^{\sharp}$ precisely $\left(\mathbf{A}, \Gamma_{f^{\sharp}}, \mathbf{B}\right)$, where $\Gamma_{f \sharp}$, the underlying $S$-sorted function of $f^{\sharp}$ is $\operatorname{Sg}_{\mathbf{A} \times \mathbf{B}}\left(\Gamma_{f}\right)$. 
We next state and prove the principle of definition by algebraic recursion for free many-sorted $\Sigma$-algebras, i.e., many-sorted DP-algebras, with respect to many-sorted partial $\Sigma$-algebras, which, for many-sorted partial algebras, is more important than the principle of definition by algebraic recursion for PDP-algebras with respect to many-sorted algebras.

Proposition 31. Let $X$ be an S-sorted set, $\mathbf{A}$ a partial $\Sigma$-algebra and $f$ an S-sorted mapping from $X$ to $A$. Then there exists a unique homomorphism

$$
f^{\partial}: \partial(f) \longrightarrow \mathbf{A}
$$

such that

1. $\eta^{X}[X] \subseteq \partial(f)$, where $\eta^{X}$ is the canonical embedding of $X$ into $\mathrm{T}_{\Sigma}(X)$.

2. $\partial(f)$ is an $X\left(\cong \eta^{X}[X]\right)$-generated relative subalgebra of $\mathbf{T}_{\Sigma}(X)$.

3. $f^{\partial} \circ$ in $^{X, \partial(f)}=f$, where in ${ }^{X, \partial(f)}$ is the canonical embedding of $X$ into $\partial(f)$.

4. $f^{\partial}$ is a closed homomorphism.

5. $\quad f^{\partial}$ is the largest homomorphic extension of $f$ to an $X$-generated relative subalgebra of $\mathbf{T}_{\Sigma}(X)$ with codomain $\mathbf{A}$.

Proof. Let $\mathbf{A}^{\infty}$ be the $\Sigma$-algebra defined as follows: The underlying $S$-sorted set of $\mathbf{A}^{\infty}$ is $\left(A_{s} \cup\left\{A_{s}\right\}\right)_{s \in S}$ and, for every $(\mathbf{s}, s) \in S^{\star} \times S$ and every $\sigma \in \Sigma_{\mathbf{s}, s}$, the operation $F_{\sigma}^{\infty}: A_{\mathbf{s}}^{\infty} \longrightarrow A_{S}^{\infty}$ associated to $\sigma$ is defined as

$$
F_{\sigma}^{\infty}\left\{\begin{aligned}
& A_{\mathbf{s}}^{\infty} \longrightarrow A_{s}^{\infty} \\
&\left(a_{j}\right)_{j \in|\mathbf{s}|} \longmapsto \begin{cases}F_{\sigma}\left(\left(a_{j}\right)_{j \in|\mathbf{s}|}\right), & \text { if }\left(a_{j}\right)_{j \in|\mathbf{s}|} \in \operatorname{Dom}\left(F_{\sigma}\right) ; \\
A_{s}, & \text { otherwise. }\end{cases}
\end{aligned}\right.
$$

We will call $\mathbf{A}^{\infty}$ the one-point per sort completion of the partial $\Sigma$-algebra $\mathbf{A}$. Notice that $\mathbf{A}$ is a relative subalgebra of $\mathbf{A}^{\infty}$, that $\mathbf{A}^{\infty}$ need not be generated by $A$ (since some of the new elements are not necessarily accessible from $A$ ) and that the canonical embedding in $A, A^{\infty}$ of $A$ into $A^{\infty}$ is the underlying mapping of a full and injective homomorphism, denoted by in $^{\mathbf{A}, \mathbf{A}^{\infty}}$, from $\mathbf{A}$ to $\mathbf{A}^{\infty}$. Then, by the universal property of the free algebra, there exists $\mathrm{a}$ unique homomorphism

$$
\left(\text { in }^{\mathbf{A}, \mathbf{A}^{\infty}} \circ f\right)^{\sharp}: \mathbf{T}_{\Sigma}(X) \longrightarrow \mathbf{A}^{\infty}
$$

such that $\left(\operatorname{in}^{\mathbf{A}, \mathbf{A}^{\infty}} \circ f\right)^{\sharp} \circ \eta^{X}=\operatorname{in}^{\mathbf{A}, \mathbf{A}^{\infty}} \circ f$. Let $\partial(f)$ be $\left(\left(\operatorname{in}^{A, A^{\infty}} \circ f\right)^{\sharp}\right)^{-1}[A]$ and let $f^{\partial}$ be $\left.\left(\text { in }^{A, A^{\infty}} \circ f\right)^{\sharp}\right|_{\left(f^{\sharp}\right)^{-1}[A]} ^{A}$, i.e., the birestriction of $\left(\operatorname{in}^{A, A^{\infty}} \circ f\right)^{\sharp}$ to $\left(\left(\operatorname{in}^{A, A^{\infty}} \circ f\right)^{\sharp}\right)^{-1}[A]$ and $A$. Then $\partial(f)$, the relative subalgebra of $\mathbf{T}_{\Sigma}(X)$ on $\partial(f)$, together with the homomorphism determined by $f^{\partial}$, which, with the customary abuse of notation, we denote by the same symbol, satisfy the desired conditions.

To better understand the mappings at play, we provide the commutative diagram in Figure 3.

Remark 31. Let us note that, by the Axiom of Regularity, we have that, for every $s \in S, A_{S} \cap$ $\left\{A_{s}\right\}=\varnothing$. Moreover, for every $(\mathbf{s}, s) \in S^{\star} \times S$ and every $\sigma \in \Sigma_{\mathbf{s}, s}, \Gamma_{F_{\sigma} \partial(f)}$, the underlying partial function of $F_{\sigma}^{\partial(f)}$, the partial operation from $\partial(f)_{\mathbf{s}}$ to $\partial(f)_{s}$ associated to $\sigma$, is

$$
\Gamma_{F_{\sigma}^{\mathbf{T}_{\Sigma}}(X)} \cap\left(\partial(f)_{\mathbf{s}} \times \partial(f)_{\mathcal{S}}\right) .
$$

On the other hand, $\Gamma_{f^{\partial}}=\operatorname{Sg}_{\mathrm{T}_{\Sigma}(X) \times \mathbf{A}}\left(\left(\eta^{X} \times \mathrm{id}_{A}\right)\left[\Gamma_{f}\right]\right)$, i.e., the underlying function of $f^{\partial}$, is the subalgebra of the partial $\Sigma$-algebra $\mathbf{T}_{\Sigma}(X) \times \mathbf{A}$ generated by the image under the S-sorted mapping $\eta^{X} \times \operatorname{id}_{A}: X \times A \longrightarrow \mathrm{T}_{\Sigma}(X) \times \mathrm{A}$, of the underlying function of $f$. Finally, we have that

1. For every $s \in S$ and every $x \in X_{S}, f_{S}^{\partial}(x)=f_{S}(x)$.

2. For every $s \in S$ and every $\sigma \in \Sigma_{\lambda, s}$, if $\operatorname{Dom}\left(F_{\sigma}\right) \neq \varnothing$, then $\sigma \in \partial(f)_{s}$ and $f_{s}^{\partial}(\sigma)=\sigma^{\mathbf{A}}$. 


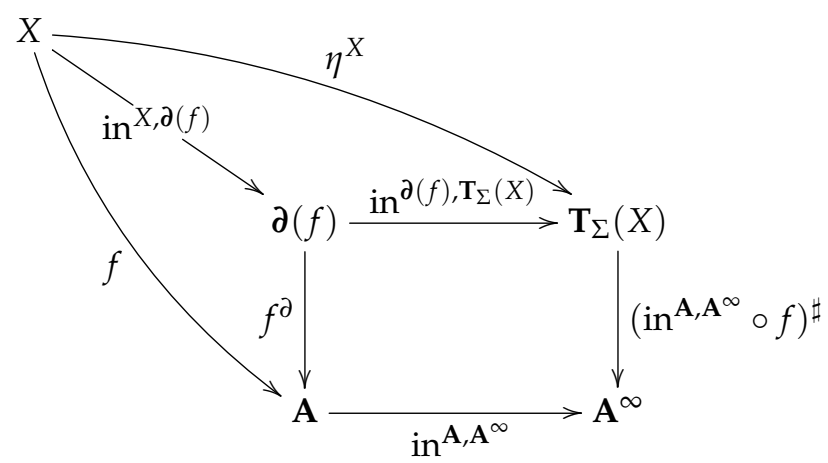

Figure 3. Recursion Theorem for DP-algebras w.r.t. partial algebras.

3. For every $(\mathbf{s}, s) \in\left(S^{\star}-\{\lambda\}\right) \times S$, every $\sigma \in \Sigma_{\mathbf{s}, s}$ and every $\left(P_{j}\right)_{j \in|\mathbf{s}|} \in \mathrm{T}_{\Sigma}(X)_{\mathbf{s}}$, if, for every $j \in|\mathbf{s}|, P_{j} \in \partial(f)_{s_{j}}$ and the family $\left(f_{s_{j}}^{\partial}\left(P_{j}\right)\right)_{j \in|\mathbf{s}|} \in \operatorname{Dom}\left(F_{\sigma}^{\mathbf{A}}\right)$, then $\sigma\left(\left(P_{j}\right)_{j \in|\mathbf{s}|}\right) \in \partial(f)_{s}$ and it holds that $\left.f_{s}^{\partial}\left(\sigma\left(\left(P_{j}\right)_{j \in|\mathbf{s}|}\right)\right)=F_{\sigma}^{\mathbf{A}}\left(\left(f_{s_{j}}^{\partial}\left(P_{j}\right)\right)\right)_{j \in|\mathbf{s}|}\right)$.

The construction that assigns to a partial $\Sigma$-algebra $\mathbf{A}$ the $\Sigma$-algebra $\mathbf{A}^{\infty}$ is not the object mapping of a functor left adjoint to the inclusion functor $\operatorname{In}_{\operatorname{Alg}(\Sigma)}$ from $\operatorname{Alg}(\Sigma)$ to $\operatorname{PAlg}(\Sigma)$. However, the just mentioned construction is the object mapping of a functor $(\cdot)^{\infty}$ from the category $\operatorname{PAlg}(\Sigma)_{c}$, of many-sorted partial $\Sigma$-algebras and closed homomorphisms, to the category $\operatorname{Alg}(\Sigma)$. For later use, specifically, when we consider completions, we next prove such a result.

Proposition 32. There exists a functor $(\cdot)^{\infty}$ from the category $\mathbf{P A l g}(\Sigma)_{\mathrm{c}}$ of many-sorted partial $\Sigma$-algebras and closed homomorphisms to the category $\operatorname{Alg}(\Sigma)$.

Proof. Let $\mathbf{A}=(A, F)$ and $\mathbf{B}=(B, G)$ be many-sorted partial $\Sigma$-algebras and $f: \mathbf{A} \longrightarrow \mathbf{B}$ a closed homomorphism. Then the many-sorted mapping $f^{\infty}$ from $A^{\infty}$ to $B^{\infty}$ defined, for every sort $s \in S$, as follows

$$
f_{s}^{\infty}\left\{\begin{aligned}
& A_{s}^{\infty} \longrightarrow B_{s}^{\infty} \\
& z \longmapsto \begin{cases}f_{s}(z), & \text { if } z \in A_{s} ; \\
B_{s}, & \text { if } z=A_{s},\end{cases}
\end{aligned}\right.
$$

is the underlying mapping of a homomorphism, also denoted by $f^{\infty}$, which we will call the one-point per sort completion of $f$, from $\mathbf{A}$ to $\mathbf{B}$. In fact, let $(\mathbf{s}, s)$ be a pair in $S^{\star} \times S, \sigma$ an operation symbol in $\Sigma_{\mathbf{s}, s}$ and $\left(z_{j}\right)_{j \in|\mathbf{s}|}$ a family of elements in $A_{\mathbf{s}}^{\infty}$. Then it could be the case that either (1) $\left(z_{j}\right)_{j \in|\mathbf{s}|} \in \operatorname{Dom}\left(F_{\sigma}^{\mathbf{A}}\right)$, or (2) $\left(z_{j}\right)_{j \in|\mathbf{s}|} \notin \operatorname{Dom}\left(F_{\sigma}^{\mathbf{A}}\right)$.

In Case (1), i.e., if $\left(z_{j}\right)_{j \in|\mathbf{s}|} \in \operatorname{Dom}\left(F_{\sigma}\right)$, then we have that $f_{\mathbf{s}}\left(\left(z_{j}\right)_{j \in|\mathbf{s}|}\right) \in \operatorname{Dom}\left(G_{\sigma}\right)$. Moreover, the following chain of equalities holds

$$
\begin{aligned}
f_{s}^{\infty}\left(F_{\sigma}^{\infty}\left(\left(z_{j}\right)_{j \in|\mathbf{s}|}\right)\right) & =f_{s}^{\infty}\left(F_{\sigma}\left(\left(z_{j}\right)_{j \in|\mathbf{s}|}\right)\right) \\
& =f_{s}\left(F_{\sigma}\left(\left(z_{j}\right)_{j \in|\mathbf{s}|}\right)\right) \\
& =G_{\sigma}\left(f_{\mathbf{s}}\left(\left(z_{j}\right)_{j \in|\mathbf{s}|}\right)\right) \\
& =G_{\sigma}\left(f_{\mathbf{s}}^{\infty}\left(\left(z_{j}\right)_{j \in|\mathbf{s}|}\right)\right) \\
& =G_{\sigma}^{\infty}\left(f_{\mathbf{s}}^{\infty}\left(\left(z_{j}\right)_{j \in|\mathbf{s}|}\right)\right) .
\end{aligned}
$$

In the just stated chain of equalities, the first equality follows from the definition of $F_{\sigma}^{\infty}$, as stated in the proof of Proposition 31; the second equality follows from the fact that, since 
$\left(z_{j}\right)_{j \in|\mathbf{s}|} \in \operatorname{Dom}\left(F_{\sigma}\right), F_{\sigma}\left(\left(z_{j}\right)_{j \in|\mathbf{s}|}\right)$ is an element of $A_{s}$ and the image under $f_{\mathcal{s}}^{\infty}$ of this last element is identical to its image under $f_{s}$; the third equality follows from the fact that, by assumption, $f$ is a homomorphism from $\mathbf{A}$ to $\mathbf{B}$; the fourth equality follows from the fact that $\left(z_{j}\right)_{j \in|\mathbf{s}|} \in \operatorname{Dom}\left(F_{\sigma}\right)$, therefore the image under $f_{S}^{\infty}$ of this last family coincides with its image under $f_{s}$; finally, the last equality follows from the fact that $f_{\mathbf{s}}\left(\left(z_{j}\right)_{j \in|\mathbf{s}|}\right) \in \operatorname{Dom}\left(G_{\sigma}\right)$, $f_{\mathbf{s}}\left(\left(z_{j}\right)_{j \in|\mathbf{s}|}\right)=f_{\mathbf{s}}^{\infty}\left(\left(z_{j}\right)_{j \in|\mathbf{s}|}\right)$ and the definition of $G_{\sigma}^{\infty}$, as stated in the proof of Proposition 31.

In Case (2), i.e., if $\left(z_{j}\right)_{j \in|\mathbf{s}|} \notin \operatorname{Dom}\left(F_{\sigma}\right)$, then, since $f$ is a closed homomorphism we have, by Definition 30, that $f_{\mathbf{s}}\left(\left(z_{j}\right)_{j \in|\mathbf{s}|}\right) \notin \operatorname{Dom}\left(G_{\sigma}\right)$. Moreover, the following chain of equalities holds

$$
\begin{aligned}
f_{s}^{\infty}\left(F_{\sigma}^{\infty}\left(\left(z_{j}\right)_{j \in|\mathbf{s}|}\right)\right) & =f_{s}^{\infty}\left(A_{s}\right) \\
& =B_{s} \\
& =G_{\sigma}^{\infty}\left(f_{\mathbf{s}}\left(\left(z_{j}\right)_{j \in|\mathbf{s}|}\right)\right) \\
& =G_{\sigma}^{\infty}\left(f_{\mathbf{s}}^{\infty}\left(\left(z_{j}\right)_{j \in|\mathbf{s}|}\right)\right) .
\end{aligned}
$$

The first equality follows from the definition of $F_{\sigma}^{\infty}$, as stated in the proof of Proposition 31, and the fact that we are assuming that $\left(z_{j}\right)_{j \in|\mathbf{s}|} \notin \operatorname{Dom}\left(F_{\sigma}^{\mathbf{A}}\right)$; the second equality follows from the definition of $f^{\infty}$; the third equality follows from the fact that $f_{\mathbf{s}}\left(\left(z_{j}\right)_{j \in|\mathbf{s}|}\right) \notin$ $\operatorname{Dom}\left(G_{\sigma}\right)$; for the fourth equality, since $f_{\mathbf{s}}\left(\left(z_{j}\right)_{j \in|\mathbf{s}|}\right) \notin \operatorname{Dom}\left(G_{\sigma}\right)$, we have that $f_{\mathbf{s}}^{\infty}\left(\left(z_{j}\right)_{j \in|\mathbf{s}|}\right) \notin$ $\operatorname{Dom}\left(G_{\sigma}\right)$, thus we have that

$$
G_{\sigma}^{\infty}\left(f_{\mathbf{s}}^{\infty}\left(\left(z_{j}\right)_{j \in|\mathbf{s}|}\right)\right)=B_{s}=G_{\sigma}^{\infty}\left(f_{\mathbf{s}}\left(\left(z_{j}\right)_{j \in|\mathbf{s}|}\right)\right)
$$

Therefore $f^{\infty}$ is a homomorphism from $\mathbf{A}^{\infty}$ to $\mathbf{B}^{\infty}$.

Let us notice that the property of being closed of $f$ has been crucial for the proof of Case (2) and cannot be weakened.

We let $(\cdot)^{\infty}$ stand for the pair of mappings that assign

1. to a partial $\Sigma$-algebra $\mathbf{A}$, the $\Sigma$-algebra $\mathbf{A}^{\infty}$, as defined in the proof of Proposition 31 ; and

2. to a closed homomorphism $f: \mathbf{A} \longrightarrow \mathbf{B}$ the homomorphism $f^{\infty}: \mathbf{A}^{\infty} \longrightarrow \mathbf{B}^{\infty}$.

We next prove that the $(\cdot)^{\infty}$ is a functor from $\operatorname{PAlg}(\Sigma)_{\mathbf{c}}$ to $\operatorname{Alg}(\Sigma)$.

Let $\mathbf{A}$ be a partial $\Sigma$-algebra. We want to prove that

$$
\left(\operatorname{id}_{\mathbf{A}}\right)^{\infty}=\mathrm{id}_{\mathbf{A}^{\infty}}
$$

i.e., that the one-point per sort completion $\left(\mathrm{id}_{\mathbf{A}}\right)^{\infty}: \mathbf{A}^{\infty} \longrightarrow \mathbf{A}^{\infty}$ of $\mathrm{id}_{\mathbf{A}}$ is identical to the identity at $\mathbf{A}^{\infty}$.

Let $s$ be a sort in $S$ and $z$ an element in $A_{S}^{\infty}$. It could be the case that either (1) $z \in A_{S}$ or (2) $z=A_{s}$.

In Case (1), i.e., if $z \in A_{S}$, then we have that

$$
\begin{aligned}
\left(\operatorname{id}_{\mathbf{A}}\right)_{s}^{\infty}(z) & =\operatorname{id}_{A_{s}}(z) \\
& =z \\
& =\left(\operatorname{id}_{\mathbf{A}^{\infty}}\right)_{s}(z)
\end{aligned}
$$

The first equality follows from the fact that $z \in A_{S}$ and the definition of $\left(\mathrm{id}_{\mathbf{A}}\right)_{S}^{\infty}(z)$; the second equality gives the value of the mapping $\left(\mathrm{id}_{\mathbf{A}}\right)_{s}$ at the element $z \in A_{s}$; and, finally, the last equality recovers the value of the mapping $\left(\mathrm{id}_{\mathbf{A}^{\infty}}\right)_{\mathcal{S}}$ at the element $z \in A_{S}^{\infty}$. 
In Case (2), i.e., if $z=A_{s}$, then we have that

$$
\begin{aligned}
\left(\operatorname{id}_{\mathbf{A}}\right)_{s}^{\infty}(z) & =\left(\operatorname{id}_{\mathbf{A}}\right)_{s}^{\infty}\left(A_{s}\right) \\
& =A_{\mathcal{S}} \\
& =\left(\operatorname{id}_{\mathbf{A}^{\infty}}\right)_{s}(z)
\end{aligned}
$$

The first equality follows from the fact that $z=A_{s}$; the second equality follows from the definition of $\left(\mathrm{id}_{\mathbf{A}}\right)_{s}^{\infty}\left(A_{s}\right)$; and, finally, the last equality recovers the value of the mapping $\left(\mathrm{id}_{\mathbf{A}^{\infty}}\right)_{s}$ at the element $z=A_{S} \in A_{S}^{\infty}$.

In any case, we conclude that $\left(\mathrm{id}_{\mathbf{A}}\right)^{\infty}=\mathrm{id}_{\mathbf{A}^{\infty}}$.

Now let $f: \mathbf{A} \longrightarrow \mathbf{B}$ and $g: \mathbf{B} \longrightarrow \mathbf{C}$ be two closed homomorphisms. Consider the one-point per sort completions $f^{\infty}: \mathbf{A}^{\infty} \longrightarrow \mathbf{B}^{\infty}$ and $g^{\infty}: \mathbf{B}^{\infty} \longrightarrow \mathbf{C}^{\infty}$. We want to prove that

$$
(g \circ f)^{\infty}=g^{\infty} \circ f^{\infty}
$$

Let $s$ be a sort in $S$ and $z$ an element in $A_{s}^{\infty}$. It could be the case that either (1) $z \in A_{s}$ or (2) $z=A_{s}$.

In Case (1), i.e., if $z \in A_{s}$, then we have that

$$
\begin{aligned}
(g \circ f)_{s}^{\infty}(z) & =(g \circ f)_{s}(z) \\
& =g_{s}\left(f_{s}(z)\right) \\
& =g_{s}^{\infty}\left(f_{s}^{\infty}(z)\right) \\
& =\left(g^{\infty} \circ f^{\infty}\right)_{s}(z) .
\end{aligned}
$$

The first equality follows from the fact that $z \in A_{s}$ and the definition of $(g \circ f)_{s}^{\infty}(z)$; the second equality unravels the $s$-sorted component of the composition of two many-sorted mappings; the third equality follows from the fact that $z \in A_{S}, f_{S}(z) \in B_{S}$ and the definitions of $f_{s}^{\infty}(z)$ and $g_{s}^{\infty}\left(f_{s}^{\infty}(z)\right)$; finally, the last equality recovers the $s$-sorted component of the composition of two many-sorted mappings.

In Case (2), i.e., if $z=A_{s}$, then the following chain of equalities holds

$$
\begin{aligned}
(g \circ f)_{s}^{\infty}(z) & =(g \circ f)_{s}^{\infty}\left(A_{s}\right) \\
& =C_{s} \\
& =g_{s}^{\infty}\left(f_{s}^{\infty}\left(A_{s}\right)\right) \\
& =\left(g^{\infty} \circ f^{\infty}\right)_{s}(z) .
\end{aligned}
$$

The first equality follows from the fact that $z=A_{s}$; the second equality follows from the definition of $(g \circ f)_{s}^{\infty}\left(A_{s}\right)$; the third equality follows from the fact that $f_{s}^{\infty}\left(A_{S}\right)=B_{S}$ and the definitions of $f_{s}^{\infty}\left(A_{S}\right)$ and $g_{s}^{\infty}\left(f_{S}^{\infty}\left(A_{S}\right)\right)$; finally, the last equality recovers the s-sorted component of the composition of two many-sorted mappings.

In any case, we conclude that $(g \circ f)^{\infty}=g^{\infty} \circ f^{\infty}$.

All in all, we conclude that $(\cdot)^{\infty}$ is a functor from $\operatorname{PAlg}(\Sigma)_{\mathrm{C}}$ to $\operatorname{Alg}(\Sigma)$.

\section{The free completion of a many-sorted partial $\Sigma$-algebra and the Schmidt construction}

We next prove, as a consequence of a well-known theorem about adjoint functors, that the inclusion functor $\operatorname{In}_{\operatorname{Alg}(\Sigma)}$ from $\operatorname{Alg}(\Sigma)$ to $\operatorname{PAlg}(\Sigma)$ has a left adjoint, the (absolutely) free completion functor. We point out that the free completion of a many-sorted partial $\Sigma$-algebra is one of the most useful tools of the theory of partial algebras. Later on, once we have defined the notion of completion and a suitable category $\operatorname{Cmpl}(\Sigma)$, of completions, we will prove that the free completion is a weakly initial object of $\mathbf{C m p l}(\Sigma)$. At the end of this section, and related to the free completion functor, we state the generalized recursion theorem of Schmidt (see [17]), which we will also call the Schmidt construction. This construction, which, as stated in the introduction, is fundamental in the field of many- 


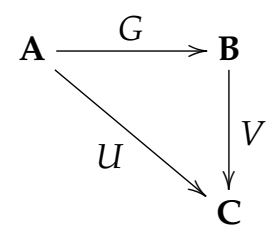

Figure 4. Conditions for the existence of a left adjoint.

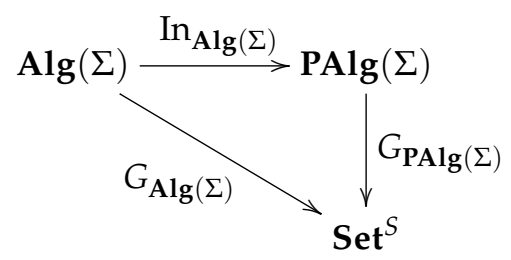

Figure 5. Conditions for the existence of the free completion.

sorted partial algebras, will be treated later from a more general point of view and, in addition, will be functorialized.

Proposition 33. If the diagram of categories and functors in Figure 4 commutes and the following conditions are satisfied:

1. A is complete, well-powered and co-well-powered,

2. G preserves limits,

3. U has a left-adjoint,

4. $V$ is faithful,

then $G$ has a left adjoint.

Corollary 4. The diagram in Figure 5 commutes and

1. $\operatorname{Alg}(\Sigma)$ is complete, well-powered and co-well-powered,

2. $\operatorname{In}_{\mathrm{Alg}(\Sigma)}$ preserves limits,

3. $G_{\mathbf{A l g}(\Sigma)}$ has a left-adjoint $\left(\mathbf{T}_{\Sigma}\right)$,

4. $G_{\operatorname{PAlg}(\Sigma)}$ is faithful.

Therefore $\operatorname{In}_{\mathrm{Alg}(\Sigma)}$ has a left adjoint $\mathbf{F}_{\Sigma}$, the free completion functor.

We next provide an explicit construction of the free completion of a partial $\Sigma$-algebra.

Proposition 34. Let $\mathbf{A}$ be a partial $\Sigma$-algebra. Then there exists a $\Sigma$-algebra $\mathbf{F}_{\Sigma}(\mathbf{A})$, the free completion of $\mathbf{A}$, and a homomorphism $\eta^{\mathbf{A}}$ from $\mathbf{A}$ to $\mathbf{F}_{\Sigma}(\mathbf{A})$ such that, for every $\Sigma$-algebra $\mathbf{B}$ and every homomorphism $f$ from $\mathbf{A}$ to $\mathbf{B}$, there exists a unique homomorphism ffc from $\mathbf{F}_{\Sigma}(\mathbf{A})$ to $\mathbf{B}$ such that $f=f^{\mathrm{fc}} \circ \eta^{\mathbf{A}}$.

Proof. Let $\mathbf{T}_{\Sigma}(A)$ be the free $\Sigma$-algebra on $A$ and, for every $(\mathbf{s}, s) \in S^{\star} \times S$ and every $\sigma \in \Sigma_{\mathbf{s}, s}$, let $\bar{F}_{\sigma}$ be the mapping from $\mathrm{T}_{\Sigma}(A)_{\mathbf{s}}$ to $\mathrm{T}_{\Sigma}(A)_{s}$ defined as:

$$
\bar{F}_{\sigma} \begin{cases}\mathrm{T}_{\Sigma}(A)_{\mathbf{s}} \longrightarrow \mathrm{T}_{\Sigma}(A)_{s} & \\ \left(P_{j}\right)_{j \in|\mathbf{s}|} \longmapsto \begin{cases}F_{\sigma}^{\mathbf{A}}\left(\left(P_{j}\right)_{j \in|\mathbf{s}|}\right), & \text { if }\left(P_{j}\right)_{j \in|\mathbf{s}|} \in \operatorname{Dom}\left(F_{\sigma}^{\mathbf{A}}\right) ; \\ F_{\sigma}^{\mathbf{T}_{\Sigma}(A)}\left(\left(P_{j}\right)_{j \in|\mathbf{s}|}\right), & \text { otherwise. }\end{cases} \end{cases}
$$

Thus $\bar{F}_{\sigma}$ has been obtained by activating $F_{\sigma}^{\mathbf{A}}$, the partial operation of $\mathbf{A}$ associated to $\sigma$, when this is possible, and when this is not the case, retrieving a syntactic term. Let $\overline{\mathbf{T}}_{\Sigma}(\mathbf{A})$ be the resulting $\Sigma$-algebra. Then $\mathbf{F}_{\Sigma}(\mathbf{A})=\mathbf{S g}_{\overline{\mathbf{T}}_{\Sigma}(\mathbf{A})}(A)$, the subalgebra of $\overline{\mathbf{T}}_{\Sigma}(\mathbf{A})$ 
generated by $A$, together with $\eta^{\mathbf{A}}$, the canonical embedding of $\mathbf{A}$ into $\mathbf{F}_{\Sigma}(\mathbf{A})$ induced by the corestriction of the mapping $\eta^{A}: A \longrightarrow \mathrm{T}_{\Sigma}(A)$ to the underlying $S$-sorted set of $\mathbf{F}_{\Sigma}(\mathbf{A})$, is a universal morphism from $\mathbf{A}$ to $\operatorname{In}_{\mathrm{Alg}(\Sigma)}$. In fact, from $\mathbf{F}_{\Sigma}(\mathbf{A})$ we obtain a partial DedekindPeano $\Sigma$-algebra $\mathbf{F}_{\Sigma}^{*}(\mathbf{A})=\left(\mathrm{F}_{\Sigma}(\mathbf{A}), F^{*}\right)$ by defining, for every $(\mathbf{s}, s) \in S^{\star} \times S$ and every $\sigma \in \Sigma_{\mathbf{s}, s}, F_{\sigma}^{*}$ to be the partial mapping from $\mathrm{F}_{\Sigma}(\mathbf{A})_{\mathbf{s}}$ to $\mathrm{F}_{\Sigma}(\mathbf{A})_{s}$ whose domain of definition is $\mathrm{F}_{\Sigma}(\mathbf{A})_{\mathbf{s}}-\operatorname{Dom}\left(F_{\sigma}^{\mathbf{A}}\right)$. Then, by Proposition 30, there exists a unique homomorphism $f^{\sharp}$ from $\mathbf{F}_{\Sigma}^{*}(\mathbf{A})$ to $\mathbf{B}$ such that $f^{\sharp} \circ \eta^{\mathbf{A}}=f$. We recall that $\Gamma_{f^{\sharp}}=\operatorname{Sg}_{\mathbf{F}_{\Sigma}^{*}(\mathbf{A}) \times \mathbf{B}}\left(\Gamma_{f}\right)$. Since $f^{\sharp} \circ \eta^{\mathbf{A}}=f$ is a homomorphism from $\mathbf{A}$ to $\mathbf{B}, f^{\sharp}$ is a homomorphism from $\mathbf{F}_{\Sigma}(\mathbf{A})$ to $\mathbf{B}$. Then it suffices to take as $f^{\text {fc }}$ the homomorphism $f^{\sharp}$ but considered as a homomorphism from $\mathbf{F}_{\Sigma}(\mathbf{A})$ to $\mathbf{B}$.

Corollary 5. The functor $\mathbf{F}_{\Sigma}$ from $\mathbf{P A} \lg (\Sigma)$ to $\mathbf{A} \lg (\Sigma)$, which sends a partial $\Sigma$-algebra $\mathbf{A}$ to $\mathbf{F}_{\Sigma}(\mathbf{A})$ and a homomorphism $f$ from $\mathbf{A}$ to $\mathbf{B}$ to $f^{@}$, the unique homomorphism $\left(\eta_{\mathbf{A}} \circ f\right)^{\mathrm{fc}}$ from $\mathbf{F}_{\Sigma}(\mathbf{A})$ to $\mathbf{F}_{\Sigma}(\mathbf{B})$ such that $\left(\eta^{\mathbf{B}} \circ f\right)^{\mathrm{fc}} \circ \eta^{\mathbf{A}}=\eta^{\mathbf{B}} \circ f$, is a left adjoint of the functor $\operatorname{In}_{\mathbf{A l g}(\Sigma)}$ from $\operatorname{Alg}(\Sigma)$ to $\mathbf{P A} \lg (\Sigma)$.

Remark 32. For every partial $\Sigma$-algebra $\mathbf{A}$, the injective homomorphism $\eta^{\mathbf{A}}$ from $\mathbf{A}$ to $\mathbf{F}_{\Sigma}(\mathbf{A})$, i.e., the value of the unit of the adjuction at $\mathbf{A}$, is, in addition, an epimorphism, hence a bimorphism. Therefore, the full subcategory $\operatorname{Alg}(\Sigma)$ of $\operatorname{PAlg}(\Sigma)$ is monoreflective and epireflective.

For later use, specifically, when we consider completions, we next show that there exists a natural transformation from the restriction of the functor $\mathbf{F}_{\Sigma}$ to $\operatorname{PAlg}(\Sigma)_{\mathrm{c}}$ to the functor $(\cdot)^{\infty}$ from $\operatorname{PAlg}(\Sigma)_{\mathrm{C}}$ to $\operatorname{Alg}(\Sigma)$.

Proposition 35. There exists a natural transformation from $\mathbf{F}_{\Sigma} \circ \operatorname{In} \operatorname{PAlg}_{(\Sigma)_{c}}$, the restriction of the functor $\mathbf{F}_{\Sigma}$ to $\mathbf{P A} \lg (\Sigma)_{\mathrm{c}}$, to the functor $(\cdot)^{\infty}$ from $\mathbf{P A} \lg (\Sigma)_{\mathrm{C}}$ to $\operatorname{Alg}(\Sigma)$.

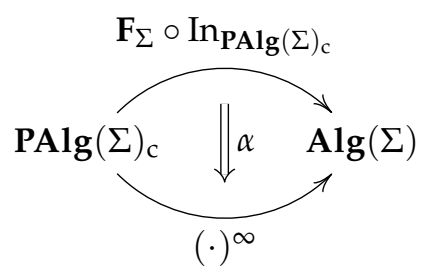

Proof. Let $\mathbf{A}=(A, F)$ be a many-sorted partial $\Sigma$-algebra. Consider $\mathbf{F}_{\Sigma}(\mathbf{A})$, the free completion of $\mathbf{A}$, and $\mathbf{A}^{\infty}$, the one-point per sort completion of $\mathbf{A}$ and the canonical embedding

$$
\operatorname{in}^{\mathbf{A}, \mathbf{A}^{\infty}}: \mathbf{A} \longrightarrow \mathbf{A}^{\infty}
$$

which is a full and injective homomorphism. Then, by the universal property of the free completion stated in Proposition 34, there exists a unique homomorphism

$$
\left(\text { in }^{\mathbf{A}, \mathbf{A}^{\infty}}\right)^{\mathrm{fc}}: \mathbf{F}_{\Sigma}(\mathbf{A}) \longrightarrow \mathbf{A}^{\infty}
$$

such that the following diagram commutes

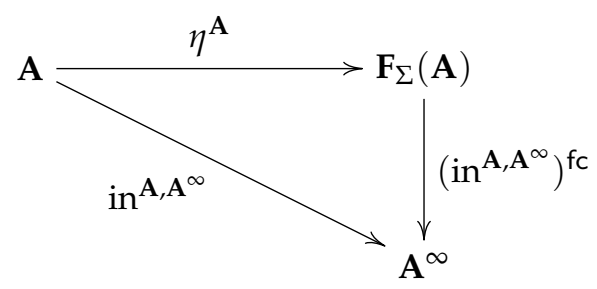

We shall let $\alpha^{\mathbf{A}}$ stand for $\left(\operatorname{in}^{\mathbf{A}, \mathbf{A}^{\infty}}\right)^{\mathrm{fc}}$. 
To show that $\alpha=\left(\alpha^{\mathbf{A}}\right)_{\mathbf{A} \in \mathbf{P A l g}(\Sigma)_{\mathrm{c}}}$ is a natural transformation from $\mathbf{F}_{\Sigma}$ to $(\cdot)^{\infty}$ we need to check that, for every closed homomorphism $f: \mathbf{A} \longrightarrow \mathbf{B}$,

$$
\alpha^{\mathbf{B}} \circ f^{@}=f^{\infty} \circ \alpha^{\mathbf{A}}
$$

i.e., that the following diagram

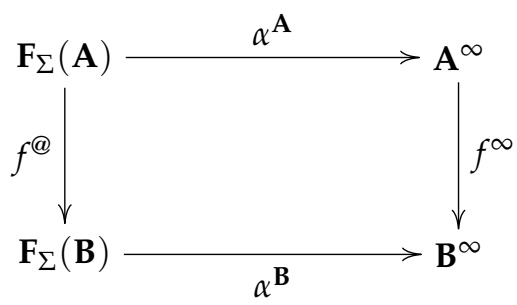

commutes.

Since, by Proposition $34, \mathrm{~F}_{\Sigma}(\mathbf{A})=\mathrm{Sg}_{\overline{\mathrm{T}}_{\Sigma}(\mathbf{A})}(A)$ it suffices to check that

$$
\left(\alpha^{\mathbf{B}} \circ f^{@}\right) \circ \eta^{\mathbf{A}}=\left(f^{\infty} \circ \alpha^{\mathbf{A}}\right) \circ \eta^{\mathbf{A}} .
$$

From the left hand side of $(+)$ we obtain the following chain of equalities

$$
\begin{aligned}
\left(\alpha^{\mathbf{B}} \circ f^{@}\right) \circ \eta^{\mathbf{A}} & =\alpha^{\mathbf{B}} \circ\left(f^{@} \circ \eta^{\mathbf{A}}\right) \\
& =\alpha^{\mathbf{B}} \circ\left(\eta^{\mathbf{B}} \circ f\right) \\
& =\left(\alpha^{\mathbf{B}} \circ \eta^{\mathbf{B}}\right) \circ f \\
& =\operatorname{in}^{\mathbf{B}, \mathbf{B}^{\infty} \circ f .}
\end{aligned}
$$

In the just stated chain of equalities the first equality follows by the associativity of the composition of S-sorted mappings; the second equality follows from Corollary 5; the third equality follows by the associativity of the composition of $S$-sorted mappings; and, finally, the last equality follows from the universal property of the free completion stated in Proposition 34 for the homomorphism in $\mathbf{B}^{\mathbf{B}} \mathbf{B}^{\infty}$.

From the right hand side of $(\dagger)$ we obtain the following chain of equalities

$$
\begin{aligned}
\left(f^{\infty} \circ \alpha^{\mathbf{A}}\right) \circ \eta^{\mathbf{A}} & =f^{\infty} \circ\left(\alpha^{\mathbf{A}} \circ \eta^{\mathbf{A}}\right) \\
& =f^{\infty} \circ \text { in }^{\mathbf{A}, \mathbf{A}^{\infty}} .
\end{aligned}
$$

In the just stated chain of equalities the first equality follows by the associativity of the composition of $S$-sorted mappings and the last equality follows from the universal property of the free completion stated in Proposition 34 for the homomorphism in ${ }^{\mathbf{A}, \mathbf{A}^{\infty}}$.

Therefore, in order to check that $(+)$ holds it suffices to check that

$$
\text { in }^{\mathbf{B}, \mathbf{B}^{\infty}} \circ f=f^{\infty} \circ \text { in }^{\mathbf{A}, \mathbf{A}^{\infty}} \text {. }
$$

Let $s$ be a sort in $S$ and $a \in A_{s}$, then we have the following chain of equalities

$$
\begin{aligned}
& \left(f^{\infty} \circ \operatorname{in}^{\mathbf{A}, \mathbf{A}^{\infty}}\right)_{s}(a)=f_{s}^{\infty}\left(\operatorname{in}_{s}^{\mathbf{A}, \mathbf{A}^{\infty}}(a)\right) \\
& =f_{s}^{\infty}(a) \\
& =f_{s}(a) \\
& =\operatorname{in}_{s}^{\mathbf{B}, \mathbf{B}^{\infty}}\left(f_{s}(a)\right) \\
& =\left(\operatorname{in}^{\mathbf{B}, \mathbf{B}^{\infty}} \circ f\right)_{s}(a) \text {. }
\end{aligned}
$$




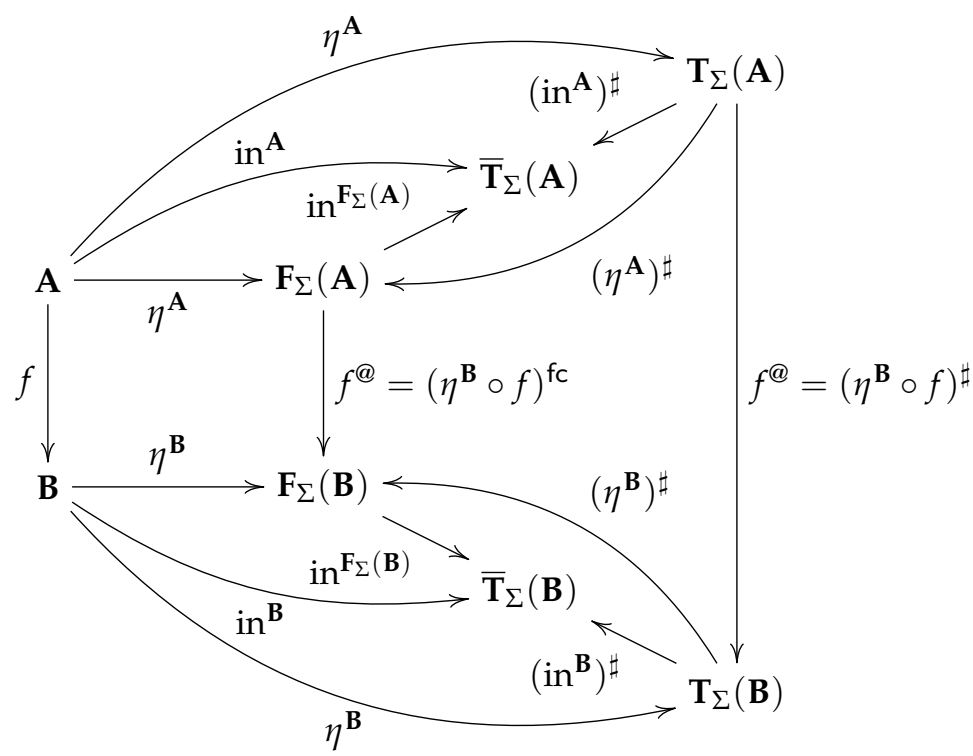

Figure 6. The free completion functor.

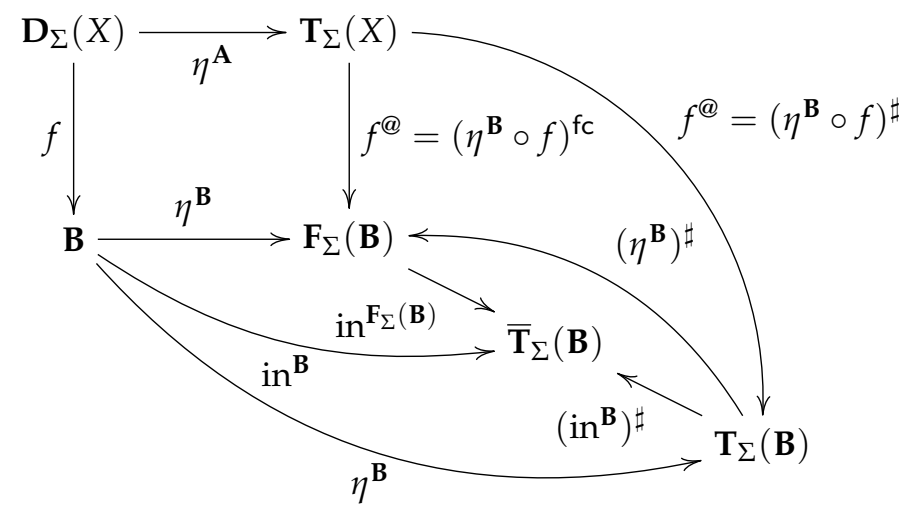

Figure 7. The free completion functor on a discrete $\Sigma$-algebra.

In the just stated chain of equalities, the first equality applies the $s$-th component of the composition of two $S$-sorted mappings; the second equality applies the $s$-th component of the inclusion mapping on an element in $A_{s}$; the third equality follows from the definition of $f^{\infty}$ in the proof of Definition 32; the fourth equality recovers the image of the $s$-th component of the inclusion mapping on an element in $B_{s}$; finally, the last equality recovers the $s$-th component of the composition of two $S$-sorted mappings.

It follows that $(\ddagger)$ holds.

Thus, $\alpha$ is a natural transformation from $\mathbf{F}_{\Sigma} \circ \operatorname{In}_{\mathrm{PAlg}(\Sigma)_{\mathrm{c}}}$ to $(\cdot)^{\infty}$.

Remark 33. Let $X$ be an $S$-sorted set. Then, for $\mathbf{D}_{\Sigma}(X)$, the discrete many-sorted partial $\Sigma$-algebra associated to an S-sorted set $X$, the three many-sorted $\Sigma$-algebras $\mathbf{F}_{\Sigma}\left(\mathbf{D}_{\Sigma}(X)\right), \overline{\mathbf{T}}_{\Sigma}\left(\mathbf{D}_{\Sigma}(X)\right)$ and $\mathbf{T}_{\Sigma}\left(\mathbf{D}_{\Sigma}(X)\right)$ are equal to $\mathbf{T}_{\Sigma}(X)$, the free many-sorted $\Sigma$-algebra on $X$. Moreover, for every manysorted partial $\Sigma$-algebra $\mathbf{B}$, we have that every mapping $f$ from $X$ to the underlying $S$-sorted set of $\mathbf{B}$ is a homomorphism $f$ from $\mathbf{D}_{\Sigma}(X)$ to $\mathbf{B}$.

To better understand the working of the functor $\mathbf{F}_{\Sigma}$, we provide the commutative diagram in Figure 6. Let us note that for $f: \mathbf{D}_{\Sigma}(X) \longrightarrow \mathbf{B}$ the diagram in Figure 6 becomes the commutative diagram in Figure 7. 


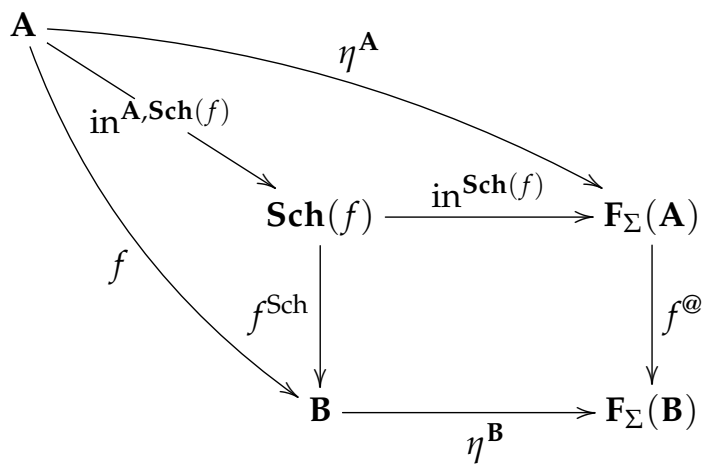

Figure 8. The Schmidt homomorphisn relative to a partial algebra.

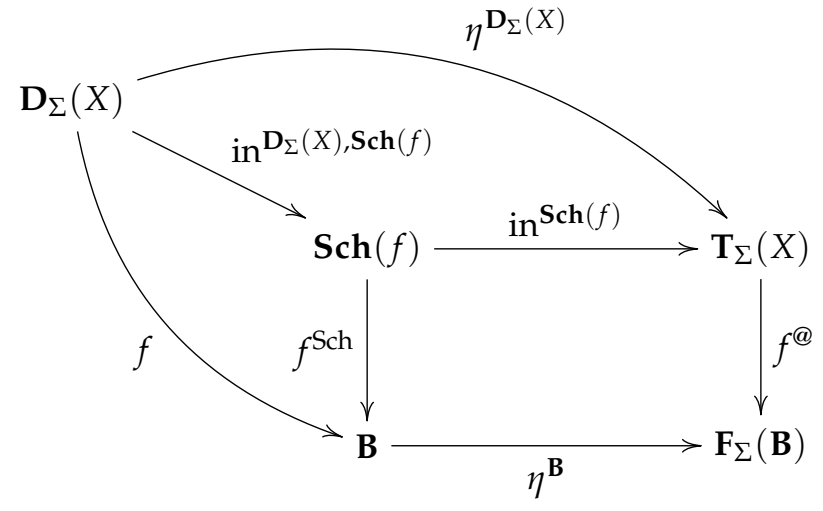

Figure 9. The Schmidt homomorphism for the discrete case.

Remark 34. If $\mathbf{A}$ is a $\Sigma$-algebra, then $\mathbf{A}$ together with $\mathrm{id}_{\mathbf{A}}$ is a free completion of $\mathbf{A}$. Moreover, for every S-sorted set $X, \mathbf{T}_{\Sigma}(X)$ together with $\eta^{X}$ is a free completion of $\mathbf{D}_{\Sigma}(X)$.

In the following proposition we state that the free completion of a partial $\Sigma$-algebra can also be characterized internally.

Proposition 36. Let $\mathbf{A}$ be a partial $\Sigma$-algebra and $\mathbf{B}$ a $\sum$-algebra such that $\mathbf{A}$ is a weak subalgebra of $\mathbf{B}$. Then $\mathbf{B}$ together with in $\mathbf{A}, \mathbf{B}$, the canonical embedding of $\mathbf{A}$ into $\mathbf{B}$, is a free completion of $\mathbf{A}$ if, and only if, the following conditions hold:

FC1. For every $(\mathbf{s}, s) \in S^{\star} \times S$, every $\sigma \in \Sigma_{\mathbf{s}, s}$ and every $\left(b_{i}\right)_{i \in|\mathbf{s}|} \in B_{\mathbf{s}}$, if $F_{\sigma}^{\mathbf{B}}\left(\left(b_{i}\right)_{i \in|\mathbf{s}|}\right) \in$ $A_{s}$, then $F_{\sigma}^{\mathbf{B}}\left(\left(b_{i}\right)_{i \in|\mathbf{s}|}\right)=F_{\sigma}^{\mathbf{A}}\left(\left(b_{i}\right)_{i \in|\mathbf{s}|}\right)$ (thus, in particular, $\left(b_{i}\right)_{i \in|\mathbf{s}|} \in \operatorname{Dom}\left(F_{\sigma}^{\mathbf{A}}\right)$ ). This condition, as Burmeister, in [17] on p. 80, says "means that no value of a fundamental operation which lies in $A$ 'can come from the outside' ".

FC2. For every $s \in S$, every $\sigma, \tau \in \Sigma_{\text {,s }}$, every $\left(b_{i}\right)_{i \in|\mathbf{s}|} \in B_{\mathbf{s}}$, where $\mathbf{s}=\operatorname{ar}(\sigma)$, and every $\left(c_{j}\right)_{j \in|\mathbf{t}|} \in B_{\mathbf{s}}$, where $\mathbf{t}=\operatorname{ar}(\tau)$, if $F_{\sigma}^{\mathbf{B}}\left(\left(b_{i}\right)_{i \in|\mathbf{s}|}\right)=F_{\tau}^{\mathbf{B}}\left(\left(c_{j}\right)_{j \in|\mathbf{t}|}\right) \notin A_{s}$, then $\sigma=\tau$ and $\left(b_{i}\right)_{i \in|\mathbf{s}|}=\left(c_{j}\right)_{j \in|\mathbf{t}|}$ (i.e., "outside of $\mathbf{A}$ " the second axiom of the notion of partial DedekindPeano is satisfied).

FC3. $\operatorname{Sg}_{\mathbf{B}}(A)=B$.

Remark 35. The condition FC1 in Proposition 36 entails that the weak subalgebra $\mathbf{A}$ of $\mathbf{B}$ is even a relative subalgebra of $\mathbf{B}$. Relative subalgebras satisfying the condition FC1 will be called normal according to Schmidt [17].

In connection with the functor $\mathbf{F}_{\Sigma}$ from $\operatorname{PA} \lg (\Sigma)$ to $\operatorname{Alg}(\Sigma)$ we have the following generalized recursion theorem, which we will also call the Schmidt construction. This 
fundamental construction will be later dealt with from a more general standpoint and, in addition, it will be functorialized.

Proposition 37 (The Schmidt construction). Let $f$ be a homomorphism from the partial $\Sigma$ algebra $\mathbf{A}$ to the partial $\Sigma$-algebra $\mathbf{B}$. Then there exists an A-generated relative subalgebra $\mathbf{S} \mathbf{c h}(f)$ of $\mathbf{F}_{\Sigma}(\mathbf{A})$, the free completion of $\mathbf{A}$, and a closed homomorphism $f^{\mathrm{Sch}}: \mathbf{S c h}(f) \longrightarrow \mathbf{B}$ such that the diagram in Figure 8 commutes, where in ${ }^{\mathbf{A}, \mathbf{S c h}}(f)$ is the canonical inclusion of $\mathbf{A}$ into $\mathbf{S c h}(f)$. Moreover, $f$ Sch is the largest homomorphic extension of $f$ to an A-generated relative subalgebra of $\mathbf{F}_{\Sigma}(\mathbf{A})$, and it is the only closed one of this kind. In honour of J. Schmidt, who introduced these concepts in [17], we will call $f^{\mathrm{Sch}}: \mathbf{S c h}(f) \longrightarrow \mathbf{B}$ the Schmidt closed A-initial extension of $f$ or, for simplicity, the Schmidt homomorphism of $f, \operatorname{Sch}(f)$ the Schmidt algebra of $f$, and $\operatorname{Ker}\left(f^{\mathrm{Sch}}\right)$, denoted by SKer $(f)$, the Schmidt kernel of $f$.

Proof. It suffices to take as $f^{\text {Sch }}$ precisely $\left(\mathbf{S c h}(f), \Gamma_{f}\right.$ sch, $\left.\mathbf{B}\right)$, where $\Gamma_{f}$ sch, the underlying $S$-sorted function of $f^{\mathrm{Sch}}$, is $\operatorname{Sg}_{\mathbf{F}_{\Sigma}(\mathbf{A}) \times \mathbf{B}}\left(\Gamma_{f}\right)$ and $\mathbf{S c h}(f)=\left(f^{@}\right)^{-1}[\mathbf{B}]$, where, with the customary abuse of notation, we have identified $B$ with $\eta^{\mathbf{B}}[B]$.

Remark 36. The canonical inclusion in $\mathbf{A}^{\mathbf{A}, \mathbf{S c h}(f)}$ of $\mathbf{A}$ into $\mathbf{S c h}(f)$ is an $\mathbf{A} \mathbf{l g}(\Sigma)$-extendable epimorphism, where, we recall, a homomorphism $f: \mathbf{C} \longrightarrow \mathbf{D}$ of the category $\mathbf{P A l g}(\Sigma)$ is called $\mathbf{A} \lg (\Sigma)$-extendable if, for every $\Sigma$-algebra $\mathbf{E}$ of the category $\mathbf{A} \lg (\Sigma)$ and every homomorphism $g: \mathbf{C} \longrightarrow \mathbf{E}$, there exists a homomorphism $h: \mathbf{D} \longrightarrow \mathbf{E}$ such that $h \circ f=g$.

From Proposition 37 we obtain, for a homomorphism whose source is a discrete many-sorted partial algebra, the following corollary.

Corollary 6. Let $X$ be an S-sorted set, B a partial $\sum$-algebra and $f$ an S-sorted mapping from $X$ to $B$ or, what is equivalent, a homomorphism from $\mathbf{D}_{\Sigma}(X)$ to $\mathbf{B}$. Then there exists an $X$-generated relative subalgebra $\mathbf{S c h}(f)$ of $\mathbf{T}_{\Sigma}(X)$, the free completion of $\mathbf{D}_{\Sigma}(X)$, and a closed homomorphism $f^{\mathrm{Sch}}: \mathbf{S c h}(f) \longrightarrow \mathbf{B}$ such that the diagram in Figure 9 commutes, where in $\mathbf{D}_{\Sigma}(X), \mathbf{S c h}(f)$ is the canonical inclusion of $X$ into $\mathbf{S c h}(f)$. Moreover, $f^{\mathrm{Sch}}: \mathbf{S c h}(f) \longrightarrow \mathbf{B}$ is the largest homomorphic extension of $f$ to an $X$-generated relative subalgebra of $\mathbf{F}_{\Sigma}(X)$, and it is the only closed one of this kind.

Let us note that for $f: \mathbf{D}_{\Sigma}(X) \longrightarrow \mathbf{B}$ the diagram in Figure 8 becomes the diagram in Figure 9. Moreover, in this particular case, we have that $f^{@}=\left(\eta^{\mathbf{B}} \circ f\right)^{\sharp}$ and $\mathbf{S c h}(f)=$ $\left(\left(\eta^{\mathbf{B}} \circ f\right)^{\sharp}\right)^{-1}\left[\eta^{\mathbf{B}}[\mathbf{B}]\right]$.

Remark 37. We would like to highlight that in the proof of Proposition 37 as well as in Corollary 6, we have made use of the free completion both in the domain and in the codomain. One of the key points for the development of this work - which aims to show the functoriality of a generalization of the Schmidt construction-has been to understand that these free completions can be replaced by others with similar behavior. This has led us, after defining a suitable category of completions and morphisms between them, to state and prove a generalization of the Schmidt construction. The reader can go to Proposition 42 to become aware of the generalisation of the Schmidt construction and to check the similarities between its proof and that of Proposition 37.

\section{7. $\Sigma$-Completions}

In this section we define a category $\operatorname{Cmpl}(\Sigma)$, of $\Sigma$-completions, and prove that $\left(\operatorname{PAlg}(\Sigma), \mathbf{F}_{\Sigma}, \eta\right)$, the $\Sigma$-completion associated to the free completion functor $\mathbf{F}_{\Sigma}$ and the unit $\eta$ of the adjunction $\mathbf{F}_{\Sigma} \dashv \operatorname{In}_{\mathrm{Alg}(\Sigma)}$, is a weakly initial object of $\operatorname{Cmpl}(\Sigma)$.

Assumption 3. From now on we will assume that the category $\operatorname{PAlg}(\Sigma)$ is equipped with a factorization system $(\mathbf{E}, \mathbf{M})$. 


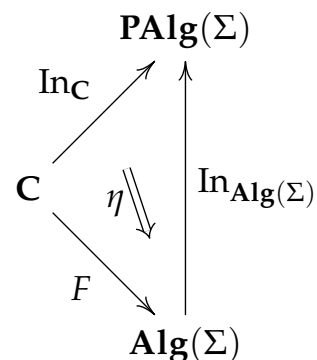

Figure 10. A $\Sigma$-completion.

Before defining the notion of $\Sigma$-completion that will be used in this paper, it is worth recalling that in [6], on p. 216, Burmeister says "... it cannot be denied that the topic of completions of partial algebras or questions about their weak, full or even normal embeddability into a total algebras of some prespecified kind are very important, in particular in computer science, but also in mathematics in general", and in [6], on p. 217, Burmeister says that a $\Sigma$-algebra $\mathbf{B}$ is a weak completion of a partial $\Sigma$-algebra $\mathbf{A}$, if $\mathbf{A}$ is a weak subalgebra of $\mathbf{B}$, a completion, if $\mathbf{A}$ is a relative subalgebra of $\mathbf{B}$, and a normal completion, if $\mathbf{A}$ is a normal in $\mathbf{B}$ (for the notion of normal subalgebra see Remark 35). If A generates its (weak) completion B, then Burmeisteir says that B is a minimal (weak or normal) completion of $\mathbf{A}$.

Definition 38 ( $\Sigma$-completion). A $\Sigma$-completion is an ordered triple $(\mathbf{C}, F, \eta)$, depicted in Figure 10 , where

1. $\mathbf{C}$ is a wide subcategory of $\mathbf{P A l g}(\Sigma)$, i.e., a subcategory of $\mathbf{P A l g}(\Sigma)$ such that $\mathrm{Ob}(\mathbf{C})=$ $\mathrm{Ob}(\mathbf{P A l g}(\Sigma))$, satisfying that

1.1 for every homomorphism $f$ of $\mathbf{C}$ if $f=h \circ g$, for some $(g, h) \in \mathbf{E} \times \mathbf{M}$, then $h$ is a homomorphism of $\mathbf{C}$.

2. F is a functor from $\mathbf{C}$ to $\mathbf{A} \lg (\Sigma)$.

3. $\eta$ is a natural transformation from $\operatorname{In}_{\mathbf{C}}$ to $\operatorname{In}_{\mathbf{A l g}(\Sigma)} \circ F$, i.e., for every homomorphism $f: \mathbf{A} \longrightarrow \mathbf{B}$ between partial $\Sigma$-algebras of $\mathbf{C}$, we have that

$$
F(f) \circ \eta^{\mathbf{A}}=\eta^{\mathbf{B}} \circ f
$$

satisfying that

3.1 for every partial $\Sigma$-algebra $\mathbf{A}, \eta^{\mathbf{A}}: \mathbf{A} \longrightarrow F(\mathbf{A})$ is a dense injective homomorphism.

Although in the definition of the notion of $\Sigma$-completion the first coordinate is univocally determined by the second, since it is the domain of the latter, and is therefore redundant, we prefer, for the sake of clarity in the formal definition, to keep it.

We warn the reader that to simplify notation from now on we will write $\mathscr{F}, \mathscr{F}_{0}, \ldots, \mathscr{G}$, $\mathscr{G}_{0}, \ldots, \mathscr{H}, \mathscr{H}_{0}, \ldots$ instead of $(\mathbf{C}, F, \eta),\left(\mathbf{C}_{0}, F_{0}, \eta_{0}\right), \ldots,(\mathbf{D}, G, \rho),\left(\mathbf{D}_{0}, G_{0}, \rho_{0}\right), \ldots,(\mathbf{E}, H, \tau)$, $\left(\mathbf{E}_{0}, H_{0}, \tau_{0}\right), \ldots$

Remark 38. The conditions set forth in the third item of Definition 38 state, using the terminology of Eilenberg $\mathcal{E} M a c$ Lane [9], that $\operatorname{In}_{\mathbf{C}}$ is a subfunctor of the functor $\operatorname{In}_{\mathrm{Alg}(\Sigma)} \circ F$. Regarding the use of the term "completion", it would be convenient to recall that the explanation of why $\widehat{\mathbb{Z}}$, the profinite completion of $\mathbb{Z}$, is a completion is that $\widehat{\mathbb{Z}}$ is equipped with a topology such that $\mathbb{Z}$ is a dense subgroup of $\widehat{\mathbb{Z}}$.

7.1. Examples of completions

Examples 1. The following ordered triples are $\Sigma$-completions. 
1. $\left(\mathbf{P A l g}(\Sigma), \mathbf{F}_{\Sigma}, \eta\right)$, where, we recall, $\mathbf{F}_{\Sigma}$ is the functor dedfined in Corollary 5 and $\eta$ the unit of the adjunction $\mathbf{F}_{\Sigma} \dashv \operatorname{In}_{\mathbf{A l g}(\Sigma)}$, where $\operatorname{In}_{\mathbf{A l g}(\Sigma)}$ is the canonical inclusion of $\operatorname{Alg}(\Sigma)$ in $\operatorname{PA} \lg (\Sigma)$.

Before setting up the second example we recall that, by Proposition 32, from $\operatorname{PAlg}(\Sigma)_{\mathrm{c}}$ to $\operatorname{Alg}(\Sigma)$ we have the functor

$$
(\cdot)^{\infty}: \operatorname{PAlg}(\Sigma)_{\mathrm{C}} \longrightarrow \operatorname{Alg}(\Sigma)
$$

2. $\quad\left(\mathbf{P A l g}(\Sigma)_{\mathrm{c}},(\cdot)^{\circ}, \iota\right)$, where $(\cdot)^{\circ}$ is the functor

$$
(\cdot)^{\circ}: \operatorname{PAlg}(\Sigma)_{\mathrm{c}} \longrightarrow \operatorname{Alg}(\Sigma)
$$

defined as follows:

1. for every partial $\sum$-algebra $\mathbf{A}, \mathbf{A}^{\circ}$ is $\mathbf{S g}_{\mathbf{A}^{\infty}}(A)$, the subalgebra of $\mathbf{A}^{\infty}$ generated by $A$, an we will call it the normal sub-one-point per sort completion of $\mathbf{A}$,

2. for every homomorphism $f: \mathbf{A} \longrightarrow \mathbf{B}$ between partial $\Sigma$-algebras, $f^{\circ}$ is the homomorphism from $\mathbf{A}^{\circ}$ to $\mathbf{B}^{\circ}$ defined as the birestriction of $f^{\infty}$ to $A^{\circ}$ and $B^{\circ}$ (this definition is correct because from $f^{\infty}[A]=f[A] \subseteq B$ and, by Remark $6, f^{\infty}\left[\operatorname{Sg}_{\mathbf{A}^{\infty}}(A)\right] \subseteq$ $\operatorname{Sg}_{\mathbf{B}^{\infty}}\left(f^{\infty}[A]\right)$ and then, taking into account that the operator $\mathrm{Sg}_{\mathbf{B}^{\infty}}$ is isotone, it follows that $f^{\infty}\left[A^{\circ}\right] \subseteq B^{\circ}$ ),

and $\iota$ the natural transformation from $\operatorname{In}_{\mathrm{PAlg}(\Sigma)_{\mathrm{c}}}$ to $\operatorname{In}_{\mathrm{Alg}(\Sigma)} \circ(\cdot)^{\circ}$ which assigns to a partial $\Sigma$-algebra A precisely in $\mathbf{A}^{\mathbf{A}} \mathbf{A}^{\circ}$, the canonical dense injective homomorphism from $\mathbf{A}$ to $\mathbf{A}^{\circ}$.

Let us notice that $(\cdot)^{\circ}$ is a subfunctor of $(\cdot)^{\infty}$ and that $\left(\operatorname{PA} \lg (\Sigma)_{\mathrm{c}},(\cdot)^{\infty}, \iota\right)$ is not a $\Sigma$-completion because it does not necessarily satisfies condition 3.1 of Definition 38.

\subsection{Morphisms between completions}

We now introduce the notion of morphism between $\Sigma$-completions.

Definition 39 (Morphisms between $\Sigma$-completions). Let $\mathscr{F}=(\mathbf{C}, F, \eta)$ and $\mathscr{G}=(\mathbf{D}, G, \rho)$ be two $\Sigma$-completions. A morphism from $\mathscr{F}$ to $\mathscr{G}$ is an ordered triple $(\mathscr{F},(K, \gamma, \alpha), \mathscr{G})$, denoted by $(K, \gamma, \alpha): \mathscr{F} \longrightarrow \mathscr{G}$ and depicted in Figure 11, where

1. $K$ is a functor from $\mathbf{D}$ to $\mathbf{C}$ (note that the direction of the functor $K$ is the opposite of that of the morphism $(K, \gamma, \alpha))$.

2. $\gamma$ is a natural transformation from $\operatorname{In}_{\mathbf{D}}$ to $\operatorname{In}_{\mathbf{C}} \circ K$, i.e., for every homomorphism $f: \mathbf{A} \longrightarrow \mathbf{B}$ of $\mathbf{D}, K(f) \circ \gamma^{\mathbf{A}}=\gamma^{\mathbf{B}} \circ f$ (see the commutative inner left diagram in Figure 12), such that

(2.1) for every many-sorted partial $\Sigma$-algebra $\mathbf{A}, \gamma^{\mathbf{A}}: \mathbf{A} \longrightarrow K(\mathbf{A})$ is a dense injective homomorphism of $\mathbf{P A} \lg (\Sigma)$.

3. $\alpha$ is a natural transformation from $F \circ K$ to $G$, i.e., for every homomorphism $f: \mathbf{A} \longrightarrow \mathbf{B}$ of $\mathbf{D}, G(f) \circ \alpha^{\mathbf{A}}=\alpha^{\mathbf{B}} \circ F(K(f))$ (see the commutative inner right diagram in Figure 12).

4. $\left(\operatorname{id}_{\operatorname{In}}{ }_{\operatorname{Alg}(\Sigma)} * \alpha\right) \circ\left(\eta * \mathrm{id}_{K}\right) \circ \gamma=\rho$, or, what is equivalent, for every many-sorted partial $\Sigma$-algebra $\mathbf{A}$, the following equality holds

$$
\alpha^{\mathbf{A}} \circ \eta^{K(\mathbf{A})} \circ \gamma^{\mathbf{A}}=\rho^{\mathbf{A}} .
$$

i.e., the upper diagram in Figure 12 commutes.

We warn the reader that to simplify notation from now on we will write $\boldsymbol{\alpha}, \boldsymbol{\alpha}_{0}, \ldots, \boldsymbol{\beta}$, $\beta_{0}, \ldots$, instead of $(K, \gamma, \alpha): \mathscr{F} \longrightarrow \mathscr{G},\left(K_{0}, \gamma_{0}, \alpha_{0}\right): \mathscr{F}_{0} \longrightarrow \mathscr{G}_{0}, \ldots,(L, \delta, \beta): \mathscr{G} \longrightarrow \mathscr{H}$, $\left(L_{0}, \delta_{0}, \beta_{0}\right): \mathscr{G}_{0} \longrightarrow \mathscr{H}_{0}, \ldots$

We next define the composition of morphisms of $\Sigma$-completions and state that the composition of morphisms of $\Sigma$-completions is a $\Sigma$-completion. 


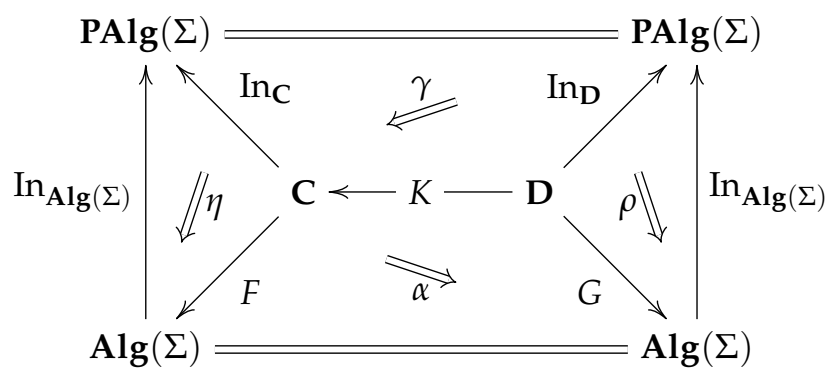

Figure 11. A morphism between $\Sigma$-completions.

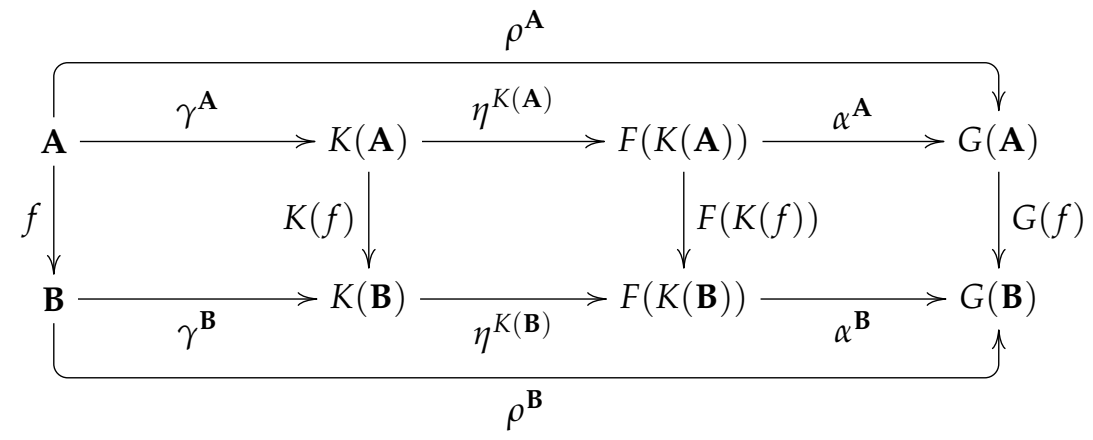

Figure 12. Detail of a morphism of $\Sigma$-completions on partial $\Sigma$-algebras.

Definition 40. Given morphisms $\alpha: \mathscr{F} \longrightarrow \mathscr{G}$ and $\beta: \mathscr{G} \longrightarrow \mathscr{H}$ between $\Sigma$-completions, its composition, denoted by $\boldsymbol{\beta} \diamond \boldsymbol{\alpha}$, is defined as

$$
\boldsymbol{\beta} \diamond \boldsymbol{\alpha}=\left(K \circ L,\left(\gamma * \mathrm{id}_{L}\right) \circ \delta, \beta \circ\left(\alpha * \mathrm{id}_{L}\right)\right),
$$

where $\mathrm{id}_{L}: L \Longrightarrow L$ is the identity natural transformation at $L$, o denotes both the composition of functors and the vertical composition of natural transformations and $*$ denotes the horizontal composition of natural transformations.

Proposition 38. Given morphisms $\alpha: \mathscr{F} \longrightarrow \mathscr{G}$ and $\beta: \mathscr{G} \longrightarrow \mathscr{H}$ between $\Sigma$-completions, then the composition, $\boldsymbol{\beta} \diamond \boldsymbol{\alpha}$, is a morphism from $\mathscr{F}$ to $\mathscr{H}$.

We next state that the composition of morphisms of $\Sigma$-completions is associative.

Proposition 39. The composition of morphism of $\Sigma$-completions is associative.

In the following proposition we state that every $\Sigma$-completion has univocally associated an identity morphism.

Proposition 40. Let $\mathscr{F}=(\mathrm{C}, F, \eta)$ be a $\sum$-completion. Then the ordered triple $\left(\operatorname{Id}_{\mathrm{C}}, \mathrm{id}_{\operatorname{In}_{\mathrm{C}}}, \mathrm{id}_{F}\right)$, denoted by $\mathscr{F}_{\mathscr{F}}$, is the identity morphism at $\mathscr{F}$.

Definition 41. We denote by $\operatorname{Cmpl}(\Sigma)$ the category of $\Sigma$-completions and morphisms between S-completions.

We next prove that $\left(\operatorname{PAlg}(\Sigma), \mathbf{F}_{\Sigma}, \eta\right)$, the free completion, is a weakly initial object of $\operatorname{Cmpl}(\Sigma)$, where, we recall, an object $x$ of a category $\mathbf{C}$ is said to be weakly initial if, for every object $y$ of $\mathbf{C}$ there exists al least one morphism from $x$ to $y$.

Proposition 41. $\left(\operatorname{PAlg}(\Sigma), \mathbf{F}_{\Sigma}, \eta\right)$ is a weakly initial object of $\mathbf{C m p l}(\Sigma)$. 
Proof. Let $(\mathbf{D}, G, \rho)$ be a $\Sigma$-completion. We will prove that there exists a unique morphism from $\left(\operatorname{PAlg}(\Sigma), \mathbf{F}_{\Sigma}, \eta\right)$ to $(\mathbf{D}, G, \rho)$ by gradually constructing it.

By Definition 38, D is a subcategory of $\operatorname{PAlg}(\Sigma)$, therefore we have the inclusion functor $\operatorname{In}_{\mathbf{D}}$ from $\mathbf{D}$ to $\operatorname{PAlg}(\Sigma)$.

Moreover, we have the identity natural transformation of the functor $\operatorname{In}_{\mathbf{D}}$, i.e., $\mathrm{id}_{\mathrm{In}_{\mathrm{D}^{\prime}}}$ which is a natural transformation from $\operatorname{In}_{\mathbf{D}}$ to $\operatorname{Id} \mathrm{IAlg}_{\mathrm{PA}(\Sigma)} \circ \operatorname{In}_{\mathbf{D}}$, this last composition being equal to $\operatorname{In}_{\mathbf{D}}$. And, for every many-sorted partial $\Sigma$-algebra $\mathbf{A}, \mathrm{id}_{I_{n_{\mathbf{D}}}}$ is such that $\left(\operatorname{id}_{I_{\mathbf{D}}}\right)^{\mathbf{A}}$, which is equal to $\mathrm{id}_{\mathbf{A}}$, is a dense injective endomorphism of $\mathbf{A}$.

We next define a natural transformation $\alpha$ from $\mathbf{F}_{\Sigma} \circ \operatorname{In}_{\mathbf{D}}$ to $G$. Let $\mathbf{A}$ be a partial $\Sigma$-algebra. Consider $G(\mathbf{A})$, the completion of $\mathbf{A}$, according to the $\Sigma$-completion $(\mathbf{D}, G, \rho)$. Then, by the universal property of the free completion, there exists a unique homomorphism

$$
\left(\rho^{\mathbf{A}}\right)^{\mathrm{fc}}: \mathbf{F}_{\Sigma}(\mathbf{A}) \longrightarrow G(\mathbf{A})
$$

such that the following diagram commutes

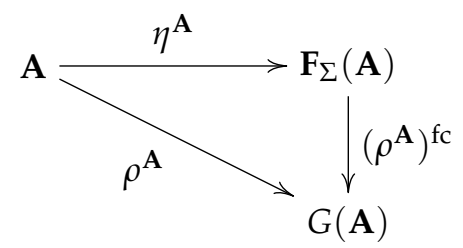

We let $\alpha^{\mathbf{A}}$ stand for $\left(\rho^{\mathbf{A}}\right)^{\text {fc }}$. Then to show that $\alpha=\left(\alpha^{\mathbf{A}}\right)_{\mathbf{A} \in \mathbf{D}}$ is a natural transformation we need to check that, for every homomorphism $f: \mathbf{A} \longrightarrow \mathbf{B}$ of $\mathbf{D}$, the following diagram in $\operatorname{Alg}(\Sigma)$ commutes

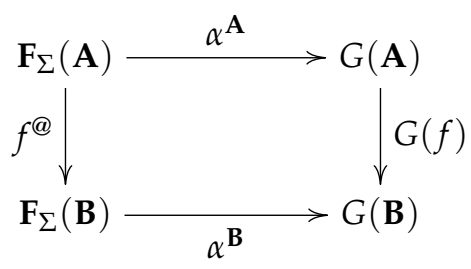

But, to check that

$$
\alpha^{\mathbf{B}} \circ f^{@}=G(f) \circ \alpha^{\mathbf{A}},
$$

since, by Proposition $34, \mathbf{F}_{\Sigma}(\mathbf{A})=\mathbf{S g}_{\overline{\mathbf{T}}_{\Sigma}(\mathbf{A})}(A)$, it suffices to check that

$$
\left(\alpha^{\mathbf{B}} \circ f^{@}\right) \circ \eta^{\mathbf{A}}=\left(G(f) \circ \alpha^{\mathbf{A}}\right) \circ \eta^{\mathbf{A}} .
$$

However, the following chain of equalities holds

$$
\begin{aligned}
\left(\alpha^{\mathbf{B}} \circ f^{@}\right) \circ \eta^{\mathbf{A}} & =\alpha^{\mathbf{B}} \circ\left(f^{@} \circ \eta^{\mathbf{A}}\right) \\
& =\alpha^{\mathbf{B}} \circ\left(\eta^{\mathbf{B}} \circ f\right) \\
& =\left(\alpha^{\mathbf{B}} \circ \eta^{\mathbf{B}}\right) \circ f \\
& =\rho^{\mathbf{B}} \circ f \\
& =G(f) \circ \rho^{\mathbf{A}} \\
& =G(f) \circ\left(\alpha^{\mathbf{A}} \circ \eta^{\mathbf{A}}\right) \\
& =\left(G(f) \circ \alpha^{\mathbf{A}}\right) \circ \eta^{\mathbf{A}} .
\end{aligned}
$$

In the just stated chain of equalities the first equality follows from the associativity of the composition of $S$-sorted mappings; the second equality follows by Corollary 5 ; the 


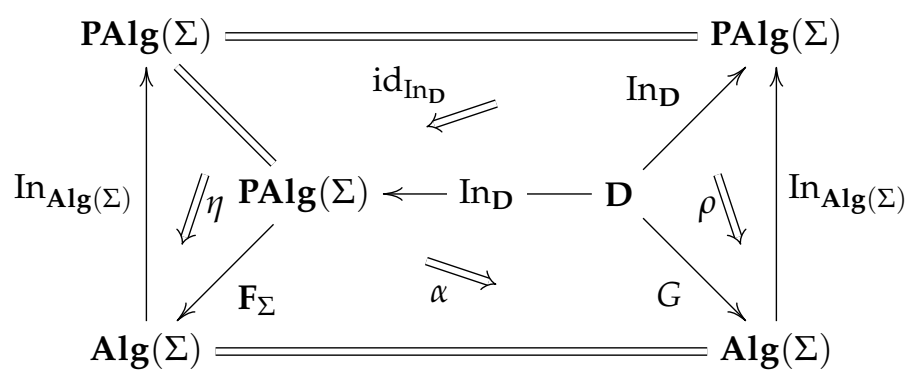

Figure 13. The free completion is a weakly initial object of $\operatorname{Cmpl}(\Sigma)$.

third equality follows from the associativity of the composition of $S$-sorted mappings; the fourth equality follows by Proposition 34; the fifth equality follows by Definition 38; the sixth equality follows by Proposition 34; and, finally, the last equality follows from the associativity of the composition of $S$-sorted mappings.

Therefore $(\dagger)$ holds.

Thus $\alpha$ is a natural transformation from $\mathbf{F}_{\Sigma} \circ \operatorname{In}_{\mathbf{D}}$ to $G$.

Finally, for every partial $\Sigma$-algebra $\mathbf{A}$, the following chain of equalities holds

$$
\alpha^{\mathbf{A}} \circ \eta^{\operatorname{In}_{\mathbf{D}}(\mathbf{A})} \circ\left(\operatorname{id}_{\operatorname{In}_{\mathbf{D}}}\right)^{\mathbf{A}}=\alpha^{\mathbf{A}} \circ \eta^{\mathbf{A}} \circ \mathrm{id}^{\mathbf{A}}=\left(\rho^{\mathbf{A}}\right)^{\mathrm{fc}} \circ \eta^{\mathbf{A}}=\rho^{\mathbf{A}} .
$$

Therefore $\left(\operatorname{In}_{\mathbf{D}}, \operatorname{id}_{\operatorname{In}_{\mathbf{D}}}, \alpha\right)$ is a morphism from $\left(\operatorname{PAlg}(\Sigma), \mathbf{F}_{\Sigma}, \eta\right)$ to $(\mathbf{D}, G, \rho)$. This morphism is depicted in the diagram of Figure 13.

Remark 39. For the $\Sigma$-completion $\left(\operatorname{PAlg}(\Sigma)_{\mathrm{c}},(\cdot)^{\circ}, \iota\right)$ (see Example 1), the morphism from

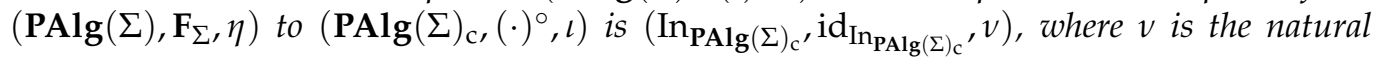
transformation from $\mathbf{F}_{\Sigma} \circ \operatorname{In}_{\mathbf{P A l g}(\Sigma)_{\mathrm{c}}}$ to $(\cdot)^{\circ}$ whose component at $\mathbf{A}$, for $\mathbf{A}$ a partial $\Sigma$-algebra, is $\left(\mathrm{in}^{\mathbf{A}, \mathbf{A}^{\circ}}\right)^{\mathrm{fc}}$, i.e., the unique homomorphism

$$
\left(\text { in }^{\mathbf{A}, \mathbf{A}^{\circ}}\right)^{\mathrm{fc}}: \mathbf{F}_{\Sigma}(\mathbf{A}) \longrightarrow \mathbf{A}^{\circ}
$$

such that the following diagram commutes

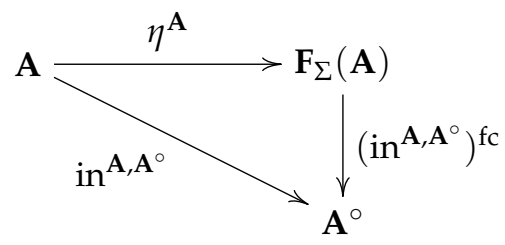

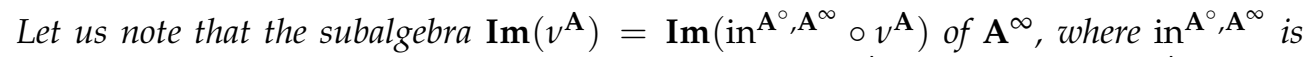
the canonical injection from $\mathbf{A}^{\circ}$ to $\mathbf{A}^{\infty}$, is such that $A \subseteq \operatorname{Im}\left(v^{\mathbf{A}}\right)$, thus, since $\operatorname{Im}\left(v^{\mathbf{A}}\right) \subseteq A^{\circ}$, $\operatorname{Im}\left(v^{\mathbf{A}}\right)=\mathbf{A}^{\circ}$. Therefore $\mathbf{F}_{\Sigma}(\mathbf{A}) / \operatorname{Ker}\left(v^{\mathbf{A}}\right) \cong \mathbf{A}^{\circ}$. Moreover, $\operatorname{Ker}\left(v^{\mathbf{A}}\right)=\Delta_{A} \cup\left(\left(\mathrm{F}_{\Sigma}(\mathbf{A})_{s}-\right.\right.$ $\left.\left.A_{S}\right)^{2}\right)_{s \in S}$. In addition, the vertical composition of the natural transformation $v$ from $\mathbf{F}_{\Sigma} \circ \operatorname{In}_{\mathbf{P A l g}(\Sigma)_{c}}$ to $(\cdot)^{\circ}$ and the natural transformation from $(\cdot)^{\circ}$ to $(\cdot)^{\infty}$ is precisely $\alpha$, the natural transformation from $\mathbf{F}_{\Sigma} \circ \operatorname{In}_{\mathbf{P A l g}(\Sigma)_{\mathrm{c}}}$ to $(\cdot)^{\infty}$ in Proposition 35.

Moreover, if a $\Sigma$-completion $(\mathbf{D}, G, \rho)$ is such that, for every $\Sigma$-algebra $\mathbf{A}$ of $\mathbf{D}, \mathbf{G}(\mathbf{A})$ is a minimal normal completion of $\operatorname{Im}\left(\rho^{\mathbf{A}}\right) \cong \mathbf{A}$ or, what is equivalente, by a result of Burmeister contained in [6] on p. 220, $\operatorname{Ker}\left(\left(\rho^{\mathbf{A}}\right)^{\mathrm{fc}}\right) \subseteq \operatorname{Ker}\left(\left(\operatorname{in}^{\mathbf{A}, \mathbf{A}^{\circ}}\right)^{\mathrm{fc}}\right)$, and $\operatorname{Im}\left(\left(\rho^{\mathbf{A}}\right)^{\mathrm{fc}}\right)=G(\mathbf{A})$, then there exists a unique homomorphism coast ${ }_{G(\mathbf{A})}\left(\left(\mathrm{in}^{\mathbf{A}, \mathbf{A}^{\circ}}\right)^{\mathrm{fc}}\right)$, the coastriction of $\left(\mathrm{in}^{\mathbf{A}, \mathbf{A}^{\circ}}\right)^{\mathrm{fc}}$ to $G(\mathbf{A})$, from $G(\mathbf{A})$ to $\mathbf{A}^{\circ}$ such that the following diagram commutes 


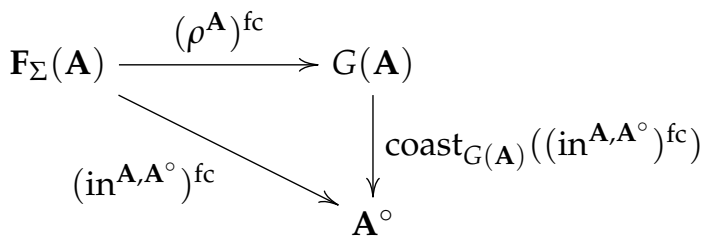

\section{The Schmidt homomorphism associated to a pair formed by a morphism of $\Sigma$-completions and a homomorphism}

In this section we begin to realize our project of showing the functionality of the Schmidt construction. To do it, we begin by associating to an ordered pair $(\boldsymbol{\alpha}, f)$, where $\alpha=(K, \gamma, \alpha)$ is a morphism of $\Sigma$-completions from $\mathscr{F}=(\mathbf{C}, F, \eta)$ to $\mathscr{G}=(\mathbf{D}, G, \rho)$ and $f$ a homomorphism of $\mathbf{D}$ from the partial $\Sigma$-algebra $\mathbf{A}$ to the partial $\Sigma$-algebra $\mathbf{B}$, a homomorphism $Y_{\alpha}^{\mathscr{G}, 0}(f): \mathbf{S c h}_{\alpha}(f) \longrightarrow \mathbf{B}$. This construction, actually, generalizes the Schmidt construction. We then prove that there exists an endofunctor, denoted $Y_{\alpha}^{\mathscr{G}, 0}$, of $\operatorname{Mor}_{\mathrm{tw}}(\mathbf{D})$, the twisted morphism category of $\mathbf{D}$, thus showing the naturalness of the Schmidt construction.

Proposition 42 (The Schmidt homomorphism). Let $\boldsymbol{\alpha}=(K, \gamma, \alpha)$ be a morphism of $\Sigma$ completions from $\mathscr{F}=(\mathbf{C}, F, \eta)$ to $\mathscr{G}=(\mathbf{D}, G, \rho)$ and $f$ a homomorphism of $\mathbf{D}$ from the partial $\Sigma$-algebra $\mathbf{A}$ to the partial $\Sigma$-algebra $\mathbf{B}$. Then there exists an $\eta^{K(\mathbf{A})}\left[\gamma^{\mathbf{A}}[A]\right]$-generated relative subalgebra $\mathbf{S} \mathbf{c h}_{\alpha}(f)$ of $F(K(\mathbf{A}))$, the $\Sigma$-completion of $K(\mathbf{A})$ associated to $\mathscr{F}$, and a homomorphism of $\mathbf{D}$ from $\mathbf{S c h}_{\alpha}(f)$ to $\mathbf{B}$, denoted by $\mathbf{Y}_{\alpha}^{\mathscr{E}, 0}(f): \mathbf{S c h}_{\alpha}(f) \longrightarrow \mathbf{B}$ such that the diagram in Figure 14 commutes, where, with the customary abuse of notation, the same symbol is used for the homomorphism $\eta^{K(\mathbf{A})} \circ \gamma^{\mathbf{A}}$ from $\mathbf{A}$ to $F(K(\mathbf{A}))$ and its corestriction to $\mathbf{S c h}_{\alpha}(f)$. Moreover, $\mathbf{Y}_{\alpha}^{\mathscr{G}, 0}(f)$ is the largest homomorphic extension of $f$ to an $\eta^{K(\mathbf{A})}\left[\gamma^{\mathbf{A}}[A]\right]$-generated relative subalgebra of $F(\mathbf{A})$.

We will call $Y_{\alpha}^{\mathscr{S}, 0}(f): \mathbf{S c h}_{\alpha}(f) \longrightarrow \mathbf{B}$ the initial extension of $f$ with respect to $\boldsymbol{\alpha}$ and $\mathscr{G}$ or the Schmidt homomorphism of $f$ with respect to $\alpha$ and $\mathscr{G}$ and $\mathbf{S c h}_{\alpha}(f)$ the Schmidt partial $\Sigma$-algebra associated to $f$ with respect to $\alpha$.

Proof. Let us recall that, since $\alpha$ is a natural transformation from $F \circ K$ to $G$, for the homomorphism $f: \mathbf{A} \longrightarrow \mathbf{B}$ of $\mathbf{D}$, the diagram in Figure 15 commutes. We let $Y_{\alpha}^{\mathscr{S}, 0}(f)$ stand for $\left(\mathbf{S c h}_{\alpha}(f), \Gamma_{Y_{\alpha}^{\mathscr{S}, 0}(f)}, \mathbf{B}\right)$, where

1. $\operatorname{Sch}_{\alpha}(f)$, the domain of $Y_{\alpha}^{\mathscr{E}, 0}(f)$, is given by $\left(G(f) \circ \alpha^{\mathbf{A}}\right)^{-1}\left[\rho^{\mathbf{B}}[B]\right]$, and

2. $\Gamma_{Y_{\alpha}^{\mathscr{E}, 0}(f)}$, the underlying $S$-sorted function of $Y_{\alpha}^{\mathscr{E}, 0}(f)$, is

$$
\operatorname{Sg}_{F(K(\mathbf{A})) \times \mathbf{B}}\left(\left(\left(\eta^{K(\mathbf{A})} \circ \gamma^{\mathbf{A}}\right) \times \operatorname{id}_{\mathbf{B}}\right)\left[\Gamma_{f}\right]\right)
$$

where $\left(\eta^{K(\mathbf{A})} \circ \gamma^{\mathbf{A}}\right) \times \mathrm{id}_{\mathbf{B}}$ is the homomorphism from $\mathbf{A} \times \mathbf{B}$ to $F(K(\mathbf{A})) \times \mathbf{B}$ that, for every $s \in S$, sends $(a, b)$ in $A_{s} \times B_{s}$ to $\left(\eta_{s}^{K(\mathbf{A})}\left(\gamma_{s}^{\mathbf{A}}(a)\right), b\right)$ in $F(K(A))_{s} \times B_{s}$.

Since $\eta^{K(\mathbf{A})}$ and $\gamma^{\mathbf{A}}$ are dense homomorphisms, the many-sorted $\Sigma$-algebra $F(K(\mathbf{A}))$ is $\eta^{K(\mathbf{A})}\left[\gamma^{\mathbf{A}}[A]\right]$-generated. Since $\mathbf{S c h}_{\alpha}(f)$ is a relative subalgebra of $F(K(\mathbf{A}))$, we conclude that $\mathbf{S c h}_{\alpha}(f)$ is also $\eta^{K(\mathbf{A})}\left[\gamma^{\mathbf{A}}[A]\right]$-generated.

We next prove that the image of the composition $\eta^{K(\mathbf{A})} \circ \gamma^{\mathbf{A}}$ is included in $\operatorname{Sch}_{\alpha}(f)$. In order to obtain this result we will prove the following equality

$$
G(f) \circ \alpha^{\mathbf{A}} \circ \eta^{K(\mathbf{A})} \circ \gamma^{\mathbf{A}}=\rho^{\mathbf{B}} \circ f .
$$

Let us note that the following chain of equalities holds

$$
\begin{aligned}
G(f) \circ \alpha^{\mathbf{A}} \circ \eta^{K(\mathbf{A})} \circ \gamma^{\mathbf{A}} & =G(f) \circ \rho^{\mathbf{A}} \\
& =\rho^{\mathbf{B}} \circ f .
\end{aligned}
$$




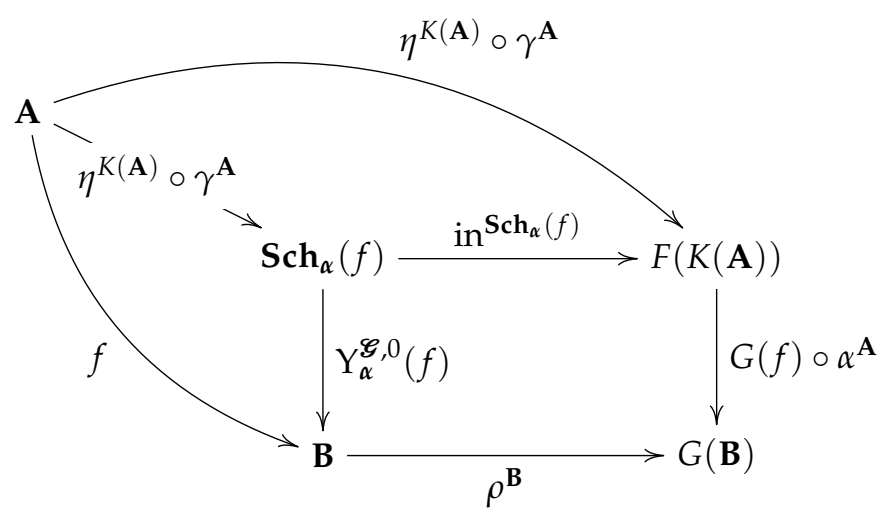

Figure 14. The Schmidt homomorphism of $f$ with respect to $\alpha$ and $\mathscr{G}$ (and relatives).

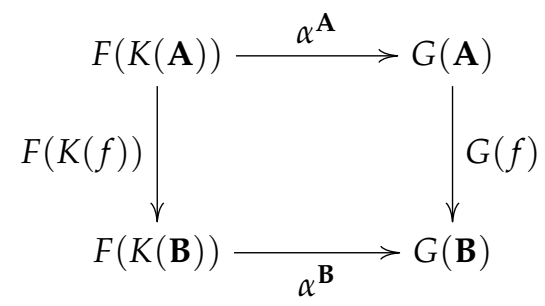

Figure 15. The natural transformation $\alpha$ at $f$.

In the just stated chain of equalities the first equality follows from the fact that $\alpha$ is a morphism from $\mathscr{F}$ to $\mathscr{G}$, thus, for every many-sorted partial $\Sigma$-algebra $\mathbf{A}$, it holds that $\alpha^{\mathbf{A}} \circ \eta^{K(\mathbf{A})} \circ \gamma^{\mathbf{A}}=\rho^{\mathbf{A}}$; finally, the last equality follows from the fact that $\rho$ is a natural transformation from $\operatorname{In}_{\mathbf{D}}$ to $\operatorname{In}_{\mathbf{A l g}(\Sigma)} \circ G$.

We conclude that the image of $\eta^{K(\mathbf{A})} \circ \gamma^{\mathbf{A}}$ is included in $\operatorname{Sch}_{(K, \gamma, \alpha)}(f)$.

According to the definition of $Y_{\alpha}^{\mathscr{G}, 0}(f)$, the next equality follows

$$
Y_{\alpha}^{\mathscr{S}, 0}(f) \circ \eta^{K(\mathbf{A})} \circ \eta^{\mathbf{A}}=f .
$$

This completes the proof.

Our next aim is to prove that there exists an endofunctor, denoted $Y_{\alpha}^{\mathscr{E}, 0}$, of $\mathbf{M o r}_{\mathrm{tw}}(\mathbf{D})$, the twisted morphism category of $\mathbf{D}$, thus showing the naturalness of the previous construction. For this purpose we first recall the definition of $\operatorname{Mor}_{\mathrm{tw}}(\mathbf{D})$.

Definition 42. Let $\mathbf{D}$ be a category. Then we denote by $\operatorname{Mor}_{\mathrm{tw}}(\mathbf{D})$ the category defined as follows

1. $\operatorname{Ob}\left(\mathbf{M o r}_{\mathrm{tw}}(\mathbf{D})\right)=\operatorname{Mor}(\mathbf{D})$, i.e., the objects of $\mathbf{M o r}_{\mathrm{tw}}(\mathbf{D})$ are the homomorphisms of $\mathbf{D}$.

2. Morphisms from the object $f: \mathbf{A} \longrightarrow \mathbf{B}$ to the object $f^{\prime}: \mathbf{A}^{\prime} \longrightarrow \mathbf{B}^{\prime}$ : the ordered triples

$\left(f,(g, h), f^{\prime}\right)$, denoted by $(g, h): f \longrightarrow f^{\prime}$, where $g$ is a homomorphism of $\mathbf{D}$ from $\mathbf{A}$ to $\mathbf{A}^{\prime}$ and h a homomorphism of $\mathbf{D}$ from $\mathbf{B}^{\prime}$ to $\mathbf{B}$ such that $f=h \circ f^{\prime} \circ g$, i.e., such that the diagram in Figure 16 commutes.

We will call $\mathbf{M o r}_{\mathrm{tw}}(\mathbf{D})$ the twisted category of morphisms of $\mathbf{D}$.

Proposition 43. Let $\boldsymbol{\alpha}=(K, \gamma, \alpha)$ be a morphism of $\Sigma$-completions from $\mathscr{F}=(\mathbf{C}, F, \eta)$ to $\mathscr{G}=(\mathbf{D}, G, \rho), f: \mathbf{A} \longrightarrow \mathbf{B}$ and $f^{\prime}: \mathbf{A}^{\prime} \longrightarrow \mathbf{B}^{\prime}$ two objects of the category $\mathbf{M o r}_{\mathrm{tw}}(\mathbf{D})$ and $(g, h)$ a morphism from $f$ to $f^{\prime}$. Then the following equation holds

$$
F(K(g)) \circ\left(\eta^{K(\mathbf{A})} \circ \gamma^{\mathbf{A}}\right)=\left(\eta^{K\left(\mathbf{A}^{\prime}\right)} \circ \gamma^{\mathbf{A}^{\prime}}\right) \circ g .
$$




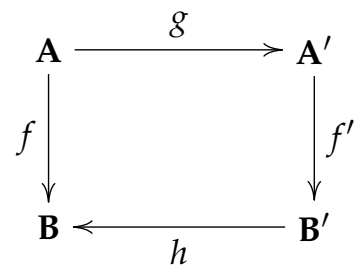

Figure 16. Morphism $(g, h)$ from $f$ to $f^{\prime}$ of the category $\operatorname{Mor}_{\mathrm{tw}}(\mathbf{D})$.

Proof. The following chain of equalities holds

$$
\begin{aligned}
& F(K(g)) \circ\left(\eta^{K(\mathbf{A})} \circ \gamma^{\mathbf{A}}\right)=\left(F(K(g)) \circ \eta^{K(\mathbf{A})}\right) \circ \eta^{\mathbf{A}} \\
& =\left(\eta^{K\left(\mathbf{A}^{\prime}\right)} \circ K(g)\right) \circ \gamma^{\mathbf{A}} \\
& =\eta^{K\left(\mathbf{A}^{\prime}\right)} \circ\left(K(g) \circ \gamma^{\mathbf{A}}\right) \\
& =\eta^{K\left(\mathbf{A}^{\prime}\right)} \circ\left(\gamma^{\mathbf{A}^{\prime}} \circ g\right) \\
& =\left(\eta^{K\left(\mathbf{A}^{\prime}\right)} \circ \gamma^{\mathbf{A}^{\prime}}\right) \circ g \text {. }
\end{aligned}
$$

In the just stated chain of equalities, the first equality follows by associativity of the composition of $S$-sorted mappings; the second equality follows from the fact that $\mathscr{F}$ is a $\Sigma$-completion, therefore $F(K(g)) \circ \eta^{K(\mathbf{A})}=\eta^{K\left(\mathbf{A}^{\prime}\right)} \circ K(g)$; the third equality follows by associativity of the composition of $S$-sorted mappings; the fourth equality follows from the fact that $\alpha$ is a morphism from $\mathscr{F}$ to $\mathscr{G}$ therefore, since $\gamma$ is a natural transformation from $\operatorname{In}_{\mathbf{D}}$ to $\operatorname{In}_{\mathbf{C}} \circ K$, it holds that $K(g) \circ \gamma^{\mathbf{A}}=\gamma^{\mathbf{A}^{\prime}} \circ g$; finally, the last equality follows by associativity of the composition of $S$-sorted mappings.

This completes the proof of Proposition 43.

Remark 40. We suggest the reader to retain the equality presented in Proposition 43 because it will be used to justify certain equalities in the proof of the following proposition. In addition, the reader will find all the homomorphisms involved in the just mentioned equality, as well as their interrelationships with other homomorphisms, in Figure 17.

We next prove that the construction associated to a morphism of $\Sigma$-completions presented in Proposition 42 is in fact functorial. But before proceeding to do so, we establish the following auxiliary definition.

Definition 43. Let $\boldsymbol{\alpha}=(K, \gamma, \alpha)$ be a morphism of $\sum$-completions from $\mathscr{F}=(\mathbf{C}, F, \eta)$ to $\mathscr{G}=(\mathbf{D}, G, \rho)$. Then we denote by $\mathrm{Y}_{\alpha}^{\mathscr{S}, 0}$ the mapping that sends

1. an object $f: \mathbf{A} \longrightarrow \mathbf{B}$ of $\mathbf{M o r}_{\mathrm{tw}}(\mathbf{D})$ to $\mathbf{Y}_{\alpha}^{\mathscr{S}, 0}(f): \mathbf{S c h}_{\alpha}(f) \longrightarrow \mathbf{B}$, which is an object of the same category, and

2. a morphism $(g, h)$ of $\mathbf{M o r}_{\mathrm{tw}}(\mathbf{D})$ from the object $f$ to the object $f^{\prime}$ to the morphism $(F(K(g)) \circ$

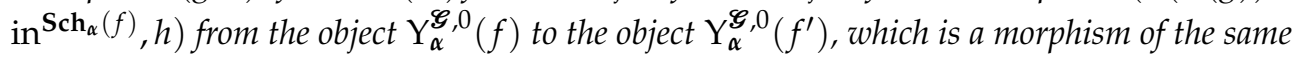
category.

The transformations associated to the morphism of $\Sigma$-completions $\boldsymbol{\alpha}$ are represented in Figure

Proposition 44. Let $\boldsymbol{\alpha}=(K, \gamma, \alpha)$ be a morphism of $\sum$-completions from $\mathscr{F}=(\mathbf{C}, F, \eta)$ to $\mathscr{G}=(\mathbf{D}, G, \rho)$. Then $\mathrm{Y}_{\alpha}^{\mathscr{G}, 0}$ is an endofunctor of $\mathbf{M o r}_{\mathrm{tw}}(\mathbf{D})$ which we will call the Schmidt endofunctor relative to $\alpha$ and $\mathscr{G}$. 


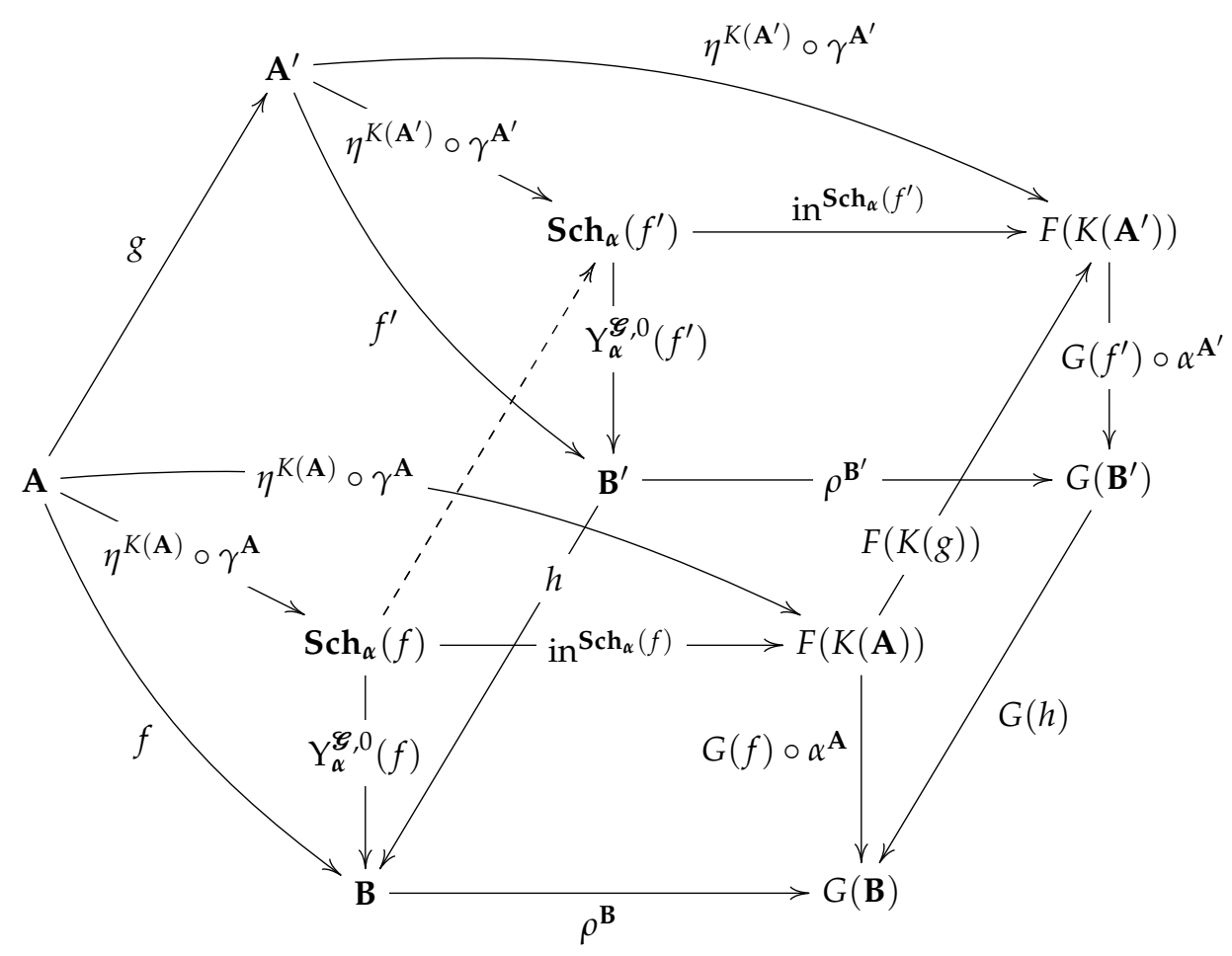

Figure 17. Pasting partial diagrams.
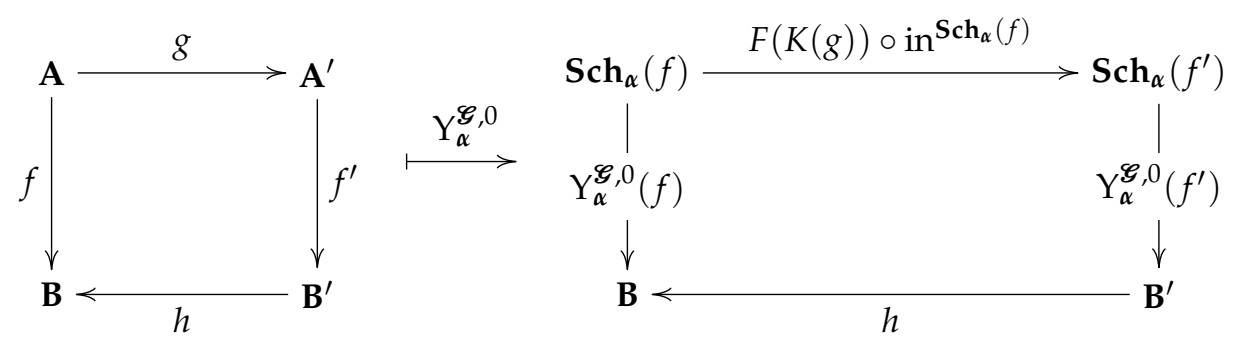

Figure 18. The functor associated to $\alpha$. 
Proof. We have divided the proof into a series of claims.

Claim 1. Let $f: \mathbf{A} \longrightarrow \mathbf{B}$ and $f^{\prime}: \mathbf{A}^{\prime} \longrightarrow \mathbf{B}^{\prime}$ be two objects of $\mathbf{M o r}_{\mathrm{tw}}(\mathbf{D})$ and $(g, h)$ a morphism from $f$ to $f^{\prime}$. Then the homomorphism $F(K(g)) \circ$ in $^{\mathbf{S c h}_{\alpha}(f)}$ from $\mathbf{S c h}_{\alpha}(f)$ to $F\left(K\left(\mathbf{A}^{\prime}\right)\right)$ is such that

$$
F(K(g))\left[\operatorname{in}^{\operatorname{Sch}_{\alpha}(f)}\left[\operatorname{Sch}_{\alpha}(f)\right]\right] \subseteq \operatorname{Sch}_{\alpha}\left(f^{\prime}\right) .
$$

In fact, by definition, we have that

$$
\operatorname{Sch}_{\alpha}\left(f^{\prime}\right)=\left(G\left(f^{\prime}\right) \circ \alpha^{\mathbf{A}^{\prime}}\right)^{-1}\left[\rho^{\mathbf{B}^{\prime}}\left[B^{\prime}\right]\right] .
$$

Hence, to prove that the image of $F(K(g)) \circ \operatorname{in}^{\operatorname{Sch}_{\alpha}(f)}$ is included in $\operatorname{Sch}_{\alpha}\left(f^{\prime}\right)$ it suffices to prove that

$$
\left(G\left(f^{\prime}\right) \circ \alpha^{\mathbf{A}^{\prime}}\right)\left[F(K(g))\left[\operatorname{in}^{\operatorname{Sch}_{\alpha}(f)}\left[\operatorname{Sch}_{\alpha}(f)\right]\right]\right] \subseteq \rho^{\mathbf{B}^{\prime}}\left[B^{\prime}\right] .
$$

But, since, by Proposition 42, the many-sorted partial $\Sigma$-algebra $\mathbf{S c h}_{\alpha}(f)$ is $\eta^{K(\mathbf{A})}\left[\gamma^{\mathbf{A}}[A]\right]$ generated and $G\left(f^{\prime}\right) \circ \alpha^{\mathbf{A}^{\prime}}, F(K(g))$ and in $^{\operatorname{Sch}_{\alpha}(f)}$ are homomorphisms, it suffices to prove that, for every sort $s \in S$ and every $a \in A_{s}$, the following statement holds

$$
\left(G\left(f^{\prime}\right) \circ \alpha^{\mathbf{A}^{\prime}}\right)_{s}\left(F(K(g))_{s}\left(\operatorname{in}_{s}^{\mathbf{S c h}_{\alpha}(f)}\left(\eta_{s}^{K(\mathbf{A})}\left(\gamma_{s}^{\mathbf{A}}(a)\right)\right)\right)\right) \in \rho_{s}^{\mathbf{B}^{\prime}}\left[B_{s}^{\prime}\right] .
$$

But we have that the following chain of equalities holds

$$
\begin{aligned}
G\left(f^{\prime}\right) \circ \alpha^{\mathbf{A}^{\prime}} \circ F(K(g)) \circ \text { in }^{\operatorname{Sch}_{\alpha}(f)} \circ \eta^{K(\mathbf{A})} \circ \gamma^{\mathbf{A}} \\
=G\left(f^{\prime}\right) \circ \alpha^{\mathbf{A}^{\prime}} \circ F(K(g)) \circ \eta^{K(\mathbf{A})} \circ \gamma^{\mathbf{A}} \\
=G\left(f^{\prime}\right) \circ \alpha^{\mathbf{A}^{\prime}} \circ \eta^{K\left(\mathbf{A}^{\prime}\right)} \circ \gamma^{\mathbf{A}^{\prime}} \circ g \\
=\rho^{\mathbf{B}^{\prime}} \circ f^{\prime} \circ g .
\end{aligned}
$$

In the just stated chain of equalities, the first equality follows from the fact that, by Proposition $42, \operatorname{in}^{\operatorname{Sch}_{\alpha}(f)} \circ \eta^{K(\mathbf{A})} \circ \gamma^{\mathbf{A}}=\eta^{K(\mathbf{A})} \circ \gamma^{\mathbf{A}}$; the second equality follows from the fact that, by Proposition 43, $F(K(g)) \circ \eta^{K(\mathbf{A})} \circ \gamma^{\mathbf{A}}=\eta^{K\left(\mathbf{A}^{\prime}\right)} \circ \gamma^{\mathbf{A}^{\prime}} \circ g$; finally, the last equality follows from the fact that, by Proposition 42, we have that $G\left(f^{\prime}\right) \circ \alpha^{\mathbf{A}^{\prime}} \circ \eta^{K\left(\mathbf{A}^{\prime}\right)} \circ \gamma^{\mathbf{A}^{\prime}}=\rho^{\mathbf{B}^{\prime}} \circ f^{\prime}$.

Therefore, to prove (Cl1) is equivalent to prove that, for every sort $s \in S$ and every $a \in A_{s}$, the following statement holds

$$
\rho_{s}^{\mathbf{B}^{\prime}}\left(f_{s}^{\prime}\left(g_{s}(a)\right)\right) \in \rho_{s}^{\mathbf{B}^{\prime}}\left[B_{s}^{\prime}\right] .
$$

But, for every sort $s \in S$ and every $a \in A_{s}$, we have that $f_{s}^{\prime}\left(g_{s}(a)\right) \in B_{s}^{\prime}$. From which it follows that $\rho_{s}^{\mathbf{B}^{\prime}}\left(f_{s}^{\prime}\left(g_{s}(a)\right)\right) \in \rho_{s}^{\mathbf{B}^{\prime}}\left[B_{s}^{\prime}\right]$. Thus we can affirm that the image of the homomorphism $F(K(g)) \circ$ in $\mathbf{S c h}_{\alpha}(f)$ from $\mathbf{S c h}_{\alpha}(f)$ to $F\left(K\left(\mathbf{A}^{\prime}\right)\right)$ is included in $\mathbf{S c h}_{\alpha}\left(f^{\prime}\right)$. To shorten notation, we let $F(K(g)) \circ$ in $^{\operatorname{Sch}_{\alpha}(f)}$ stand for $\operatorname{cores}_{\mathbf{S c h}_{\alpha}\left(f^{\prime}\right)}\left(F(K(g)) \circ \operatorname{in}^{\operatorname{Sch}_{\alpha}(f)}\right)$, the corestriction of $F(K(g)) \circ \mathrm{in}^{\mathbf{S c h}_{\alpha}(f)}$ to $\mathbf{S c h}_{\alpha}\left(f^{\prime}\right)$. This homomorphism is represented in the diagram of Figure 17 as an unnamed dashed arrow.

Claim 1 follows.

Claim 2. Let $f: \mathbf{A} \longrightarrow \mathbf{B}$ and $f^{\prime}: \mathbf{A}^{\prime} \longrightarrow \mathbf{B}^{\prime}$ be two objects of $\mathbf{M o r}_{\mathrm{tw}}(\mathbf{D})$ and $(g, h)$ a morphism

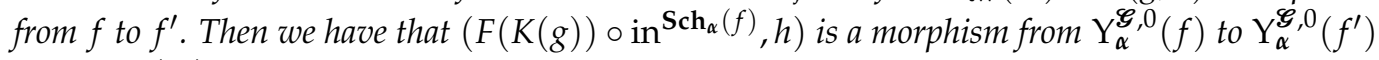
of $\operatorname{Mor}_{\mathrm{tw}}(\mathbf{D})$, i.e.,

$$
Y_{\alpha}^{\mathscr{G}, 0}(f)=h \circ Y_{\alpha}^{\mathscr{G}, 0}\left(f^{\prime}\right) \circ F(K(g)) \circ \operatorname{in}^{\operatorname{Sch}_{\alpha}(f)} .
$$

In fact, since, by Proposition 42, the many-sorted partial $\Sigma$-algebra $\mathbf{S c h}_{\alpha}(f)$ is $\eta^{K(\mathbf{A})}\left[\gamma^{\mathbf{A}}[A]\right]$ generated and $h, \mathbf{Y}_{\boldsymbol{\alpha}}^{\mathscr{S}, 0}(f), F(K(g))$ and in $\mathbf{S c h}_{\alpha}(f)$ are homomorphisms, to prove Claim 2 it 
suffices to prove that

$$
\mathrm{Y}_{\boldsymbol{\alpha}}^{\mathscr{S}, 0}(f) \circ \eta^{K(\mathbf{A})} \circ \gamma^{\mathbf{A}}=h \circ \mathrm{Y}_{\boldsymbol{\alpha}}^{\mathscr{E}, 0}\left(f^{\prime}\right) \circ F(K(g)) \circ \operatorname{in}^{\mathbf{S c h}_{\alpha}(f)} \circ \eta^{K(\mathbf{A})} \circ \gamma^{\mathbf{A}} .
$$

Starting from the right hand side of the above equation, we have that the following chain of equalities holds

$$
\begin{aligned}
& h \circ Y_{\alpha}^{\mathscr{G}, 0}\left(f^{\prime}\right) \circ F(K(g)) \circ \operatorname{in}^{\mathbf{S c h}_{\alpha}(f)} \circ \eta^{K(\mathbf{A})} \circ \gamma^{\mathbf{A}} \\
& =h \circ Y_{\alpha}^{\mathscr{G}, 0}\left(f^{\prime}\right) \circ F(K(g)) \circ \eta^{K(\mathbf{A})} \circ \gamma^{\mathbf{A}} \\
& =h \circ Y_{\alpha}^{\mathscr{G}, 0}\left(f^{\prime}\right) \circ \eta^{K\left(\mathbf{A}^{\prime}\right)} \circ \gamma^{\mathbf{A}^{\prime}} \circ g \\
& =h \circ f^{\prime} \circ g \\
& =f \\
& =Y_{\alpha}^{\mathscr{E}, 0}(f) \circ \eta^{K(\mathbf{A})} \circ \gamma^{\mathbf{A}} \text {. }
\end{aligned}
$$

In the just stated chain of equalities, the first equality follows from the fact that, by Proposition $42, \operatorname{in}^{\mathbf{S c h}_{\alpha}(f)} \circ \eta^{K(\mathbf{A})} \circ \gamma^{\mathbf{A}}=\eta^{K(\mathbf{A})} \circ \gamma^{\mathbf{A}}$; the second equality follows from the fact that, by Proposition 43, $F(K(g)) \circ \eta^{K(\mathbf{A})} \circ \gamma^{\mathbf{A}}=\eta^{K\left(\mathbf{A}^{\prime}\right)} \circ \gamma^{\mathbf{A}^{\prime}} \circ g$; the third equality follows from the fact that, by Proposition $42, Y_{\alpha}^{\mathscr{E}, 0}\left(f^{\prime}\right) \circ \eta^{K\left(\mathbf{A}^{\prime}\right)} \circ \gamma^{\mathbf{A}^{\prime}}=f^{\prime}$; the fourth equality follows from the fact that $(g, h)$ is a morphism of $\operatorname{Mor}_{\text {tw }}(\mathbf{D})$ from $f$ to $f^{\prime}$; finally, the last equality follows from the fact that, by Proposition $42, Y_{\alpha}^{\mathscr{G}, 0}(f) \circ \eta^{K(\mathbf{A})} \circ \gamma^{\mathbf{A}}=f$.

Claim 2 follows.

Therefore $Y_{\alpha}^{\mathscr{E}, 0}$ maps objects to objects and morphisms to morphisms. We next prove that $Y_{\alpha}^{\mathscr{S}, 0}$ preserves identities.

Claim 3. Let $f: \mathbf{A} \longrightarrow \mathbf{B}$ be a homomorphism between partial $\Sigma$-algebras of $\mathbf{D}$. Then

$$
\mathrm{Y}_{\alpha}^{\mathscr{E}, 0}\left(\mathrm{id}_{f}\right)=\mathrm{id}_{\mathrm{Y}_{\alpha}^{\mathscr{S}, 0}(f)} .
$$

We recall that id $f$, the identity at $f$ in $\operatorname{Mor}_{\text {tw }}(\mathbf{D})$, is $\left(\operatorname{id}_{\mathbf{A}}, \mathrm{id}_{\mathbf{B}}\right)$. Let us note, that, on the one hand, we have that

$$
\mathrm{Y}_{\alpha}^{\mathscr{G}, 0}\left(\mathrm{id}_{f}\right)=Y_{\alpha}^{\mathscr{S}, 0}\left(\mathrm{id}_{\mathbf{A}}, \operatorname{id}_{\mathbf{B}}\right)=\left(F\left(K\left(\operatorname{id}_{\mathbf{A}}\right)\right) \circ \operatorname{in}^{\operatorname{Sch}_{\alpha}(f)}, \operatorname{id}_{\mathbf{B}}\right),
$$

and, on the other hand, we have that

$$
\operatorname{id}_{Y_{\alpha}^{\mathscr{S}, 0}(f)}=\left(\operatorname{id}_{\mathbf{S c h}_{\alpha}(f)}, \operatorname{id}_{\mathbf{B}}\right) .
$$

Hence, to check that $Y_{\alpha}^{\mathscr{G}, 0}\left(\operatorname{id}_{f}\right)=\operatorname{id}_{Y_{\alpha}^{\mathscr{S}, 0}(f)}$, it suffices to prove that

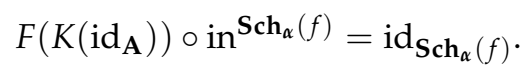

But, by Proposition 42, the many-sorted partial $\Sigma$-algebra $\mathbf{S c h}_{\alpha}(f)$ is $\eta^{K(\mathbf{A})}\left[\gamma^{\mathbf{A}}[A]\right]$ generated and $F\left(K\left(\operatorname{id}_{\mathbf{A}}\right)\right)$, in $\operatorname{Sch}_{\alpha}(f)$, and in $\operatorname{Sch}_{\alpha}(f)$ are homomorphisms. Therefore, it suffices to prove that

$$
F\left(K\left(\operatorname{id}_{\mathbf{A}}\right)\right) \circ \operatorname{in}^{\operatorname{Sch}_{\alpha}(f)} \circ \eta^{K(\mathbf{A})} \circ \gamma^{\mathbf{A}}=\operatorname{id}_{\mathbf{S c h}_{\alpha}(f)} \circ \eta^{K(\mathbf{A})} \circ \gamma^{\mathbf{A}} .
$$




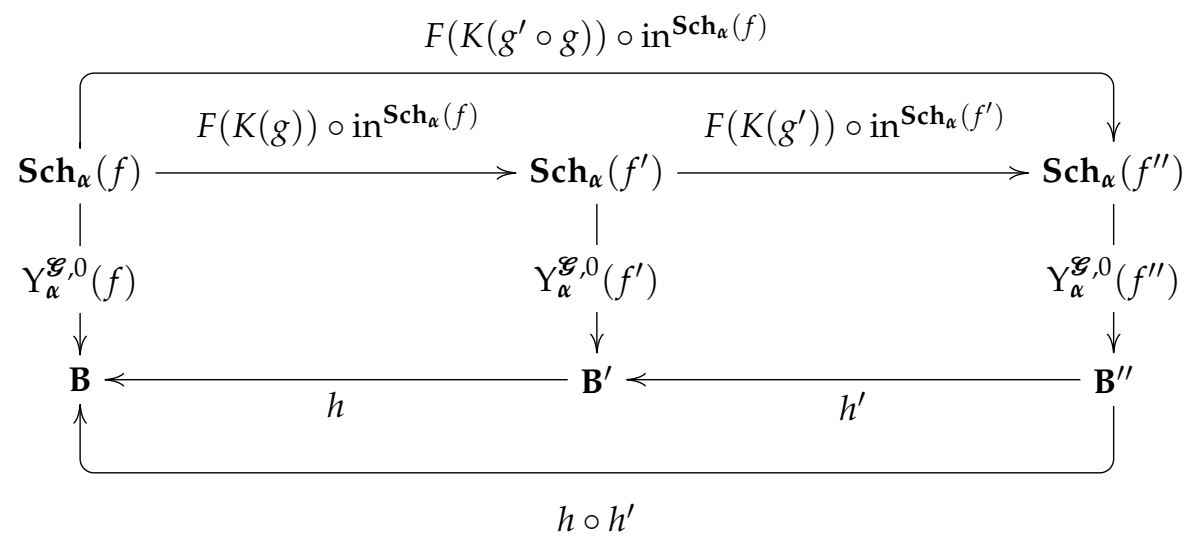

Figure 19. The functor associated to the morphism $\alpha$ on compositions.

However, the following chain of equalities holds

$$
\begin{aligned}
F\left(K\left(\mathrm{id}_{\mathbf{A}}\right)\right) \circ \operatorname{in}^{\mathbf{S c h}_{\alpha}(f)} \circ \eta^{K(\mathbf{A})} \circ \gamma^{\mathbf{A}} & =F\left(K\left(\mathrm{id}_{\mathbf{A}}\right)\right) \circ \eta^{K(\mathbf{A})} \circ \gamma^{\mathbf{A}} \\
& =\eta^{K(\mathbf{A})} \circ \gamma^{\mathbf{A}} \circ \mathrm{id}_{\mathbf{A}} \\
& =\eta^{K(\mathbf{A})} \circ \gamma^{\mathbf{A}} \\
& =\operatorname{id}_{\mathbf{S c h}_{\alpha}(f)} \circ \eta^{K(\mathbf{A})} \circ \gamma^{\mathbf{A}} .
\end{aligned}
$$

In the just stated chain of equalities, the first equality follows from the fact that, by Proposition 42, in $^{\mathbf{S c h}_{\alpha}(f)} \circ \eta^{K(\mathbf{A})} \circ \gamma^{\mathbf{A}}=\eta^{K(\mathbf{A})} \circ \gamma^{\mathbf{A}}$; the second equality follows from the fact that, by Proposition $43, F\left(K\left(\mathrm{id}_{\mathbf{A}}\right)\right) \circ \eta^{K(\mathbf{A})} \circ \gamma^{\mathbf{A}}=\eta^{K(\mathbf{A})} \circ \gamma^{\mathbf{A}} \circ \mathrm{id}_{\mathbf{A}}$; the third equality follows from the fact that $\mathrm{id}_{\mathbf{A}}$ is an identity; finally the last equality follows from the fact that, by Proposition 42, the image of $\eta^{K(\mathbf{A})} \circ \gamma^{\mathbf{A}}$ is included in $\mathbf{S c h}_{\boldsymbol{\alpha}}(f)$ and $\mathbf{i d}_{\mathbf{S c h}_{\alpha}(f)}$ is an identity.

Claim 3 follows.

We next prove that $\mathrm{Y}_{\alpha}^{\mathscr{G}, 0}$ preserves the composition of morphisms of $\operatorname{Mor}_{\mathrm{tw}}(\mathbf{D})$.

Claim 4. Let $f: \mathbf{A} \longrightarrow \mathbf{B}, f^{\prime}: \mathbf{A}^{\prime} \longrightarrow \mathbf{B}^{\prime}$ and $f^{\prime \prime}: \mathbf{A}^{\prime \prime} \longrightarrow \mathbf{B}^{\prime \prime}$ be homomorphisms between partial $\Sigma$-algebras of $\mathbf{D}$. Let $(g, h)$ and $\left(g^{\prime}, h^{\prime}\right)$ be morphisms of $\mathbf{M o r}_{\mathbf{t w}}(\mathbf{D})$ from $f$ to $f^{\prime}$ and from $f^{\prime}$ to $f^{\prime \prime}$, respectively. Then

$$
\mathrm{Y}_{\boldsymbol{\alpha}}^{\mathscr{G}, 0}\left(\left(g^{\prime}, h^{\prime}\right) \circ(g, h)\right)=\mathrm{Y}_{\alpha}^{\mathscr{S}, 0}\left(g^{\prime}, h^{\prime}\right) \circ \mathrm{Y}_{\alpha}^{\mathscr{S}, 0}(g, h)
$$

We recall that $\left(g^{\prime}, h^{\prime}\right) \circ(g, h)$, the composition of $\left(g^{\prime}, h^{\prime}\right)$ with $(g, h)$ in $\mathbf{M o r}_{\mathrm{tw}}(\mathbf{D})$, is $\left(g^{\prime} \circ g, h \circ h^{\prime}\right)$. Let us note that, on the one hand, we have that

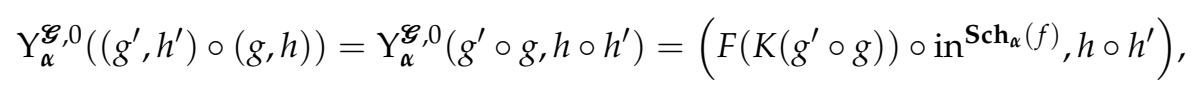

and, on the other hand, we have that

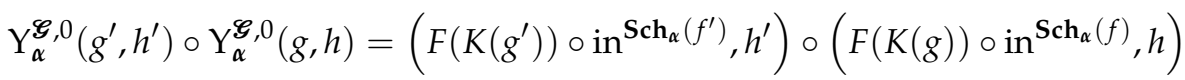

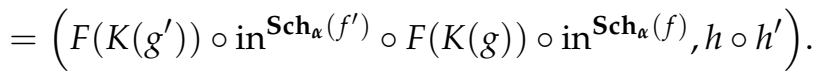

(The reader is advised to consult Figure 19 for a better understanding of the homomorphisms under consideration.)

Thus, to check that

$$
\mathrm{Y}_{\boldsymbol{\alpha}}^{\mathscr{G}, 0}\left(\left(g^{\prime}, h^{\prime}\right) \circ(g, h)\right)=\mathrm{Y}_{\boldsymbol{\alpha}}^{\mathscr{G}, 0}\left(g^{\prime}, h^{\prime}\right) \circ \mathrm{Y}_{\boldsymbol{\alpha}}^{\mathscr{G}, 0}(g, h),
$$


it suffices to prove that

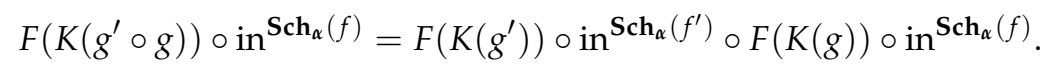

But, since, by Proposition 42, the many-sorted partial $\Sigma$-algebra $\mathbf{S c h}_{\boldsymbol{\alpha}}(f)$ is $\eta^{K(\mathbf{A})}\left[\gamma^{\mathbf{A}}[A]\right]$ generated and $F\left(K\left(g^{\prime} \circ g\right)\right)$, in $\mathbf{S c h}_{\alpha}(f), F\left(K\left(g^{\prime}\right)\right)$, in $\mathbf{S c h}_{\alpha}\left(f^{\prime}\right)$ and $F(K(g))$ are homomorphisms, it suffices to prove that

$$
\begin{aligned}
& F\left(K\left(g^{\prime} \circ g\right)\right) \circ \operatorname{in}^{\mathbf{S c h}_{\alpha}(f)} \circ \eta^{K(\mathbf{A})} \circ \gamma^{\mathbf{A}} \\
& =F\left(K\left(g^{\prime}\right)\right) \circ \operatorname{in}^{\mathbf{S c h}_{\alpha}\left(f^{\prime}\right)} \circ F(K(g)) \circ \text { in }^{\mathbf{S c h}_{\alpha}(f)} \circ \eta^{K(\mathbf{A})} \circ \gamma^{\mathbf{A}} .
\end{aligned}
$$

Now, starting from the right hand side of the above equation, we have that the following chain of equalities holds

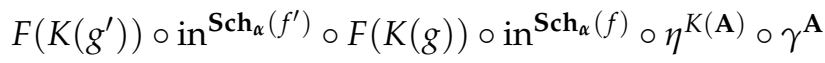

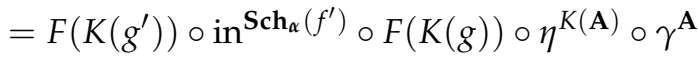

$$
\begin{aligned}
& =F\left(K\left(g^{\prime}\right)\right) \circ \operatorname{in}^{\mathbf{S c h}_{\alpha}\left(f^{\prime}\right)} \circ \eta^{K\left(\mathbf{A}^{\prime}\right)} \circ \gamma^{\mathbf{A}^{\prime}} \circ g \\
& =F\left(K\left(g^{\prime}\right)\right) \circ \eta^{K\left(\mathbf{A}^{\prime}\right)} \circ \gamma^{\mathbf{A}^{\prime}} \circ g \\
& =\eta^{K\left(\mathbf{A}^{\prime \prime}\right)} \circ \gamma^{\mathbf{A}^{\prime \prime}} \circ g^{\prime} \circ g \\
& =F\left(K\left(g^{\prime} \circ g\right)\right) \circ \eta^{K(\mathbf{A})} \circ \gamma^{\mathbf{A}} \\
& =F\left(K\left(g^{\prime} \circ g\right)\right) \circ \operatorname{in}^{\mathbf{S c h}_{\alpha}(f)} \circ \eta^{K(\mathbf{A})} \circ \gamma^{\mathbf{A}} \text {. }
\end{aligned}
$$

In the just stated chain of equalities, the first equality follows from the fact that, by Proposition 42, in $^{\mathbf{S c h}_{\alpha}(f)} \circ \eta^{K(\mathbf{A})} \circ \gamma^{\mathbf{A}}=\eta^{K(\mathbf{A})} \circ \gamma^{\mathbf{A}}$; the second equality follows from the fact that,

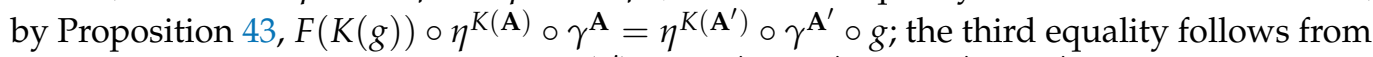
the fact that, by Proposition 42, in $\mathbf{S c h}_{\alpha}\left(f^{\prime}\right) \circ \eta^{K\left(\mathbf{A}^{\prime}\right)} \circ \gamma^{\mathbf{A}^{\prime}}=\eta^{K\left(\mathbf{A}^{\prime}\right)} \circ \gamma^{\mathbf{A}^{\prime}}$; the fourth equality follows from the fact that, by Proposition 43, $F\left(K\left(g^{\prime}\right)\right) \circ \eta^{K\left(\mathbf{A}^{\prime}\right)} \circ \gamma^{\mathbf{A}^{\prime}}=\eta^{K\left(\mathbf{A}^{\prime \prime}\right)} \circ \gamma^{\mathbf{A}^{\prime \prime}} \circ g^{\prime}$; the fifth equality follows from the fact that, by Proposition $43, F\left(K\left(g^{\prime} \circ g\right)\right) \circ \eta^{K(\mathbf{A})} \circ \gamma^{\mathbf{A}}=$ $\eta^{K\left(\mathbf{A}^{\prime \prime}\right)} \circ \gamma^{\mathbf{A}^{\prime \prime}} \circ g^{\prime} \circ g$; finally, the last equality follows from the fact that, by Proposition 42 , $\operatorname{in}^{\mathbf{S c h}_{\alpha}(f)} \circ \eta^{K(\mathbf{A})} \circ \gamma^{\mathbf{A}}=\eta^{K(\mathbf{A})} \circ \gamma^{\mathbf{A}}$.

Claim 4 follows.

This completes the proof of Proposition 44.

\section{Functoriality of the Schmidt construction}

In this section we finally reach the objective expressed in the title of the paper. Specifically, we will prove that, for every $\Sigma$-completion $\mathscr{G}$, there exists a functor $\mathrm{Y}^{\mathscr{G}}$ from the comma category $(\operatorname{Cmpl}(\Sigma) \downarrow \mathscr{G})$ to $\operatorname{End}\left(\operatorname{Mor}_{\mathrm{tw}}(\mathbf{D})\right)$, the category of endofunctors of $\operatorname{Mor}_{\text {tw }}\left(\right.$ D), such that $Y^{\mathscr{G}, 0}$, the object mapping of $Y^{\mathscr{G}}$, sends an object $\alpha: \mathscr{F} \longrightarrow \mathscr{G}$ of $(\operatorname{Cmpl}(\Sigma) \downarrow \mathscr{G})$, i.e., a morphism of $\Sigma$-completion of $\operatorname{Cmpl}(\Sigma)$ with codomain $\mathscr{G}$, to the Schmidt endofunctor $\mathrm{Y}_{\alpha}^{\mathscr{S}, 0}$ in $\operatorname{End}\left(\operatorname{Mor}_{\mathrm{tw}}(\mathbf{D})\right)$.

To obtain the result just mentioned we first recall the definition of the comma category $(\operatorname{Cmpl}(\Sigma) \downarrow \mathscr{G})$.

Definition 44. Let $\mathscr{G}$ be a $\Sigma$-completion of $\mathbf{C m p l}(\Sigma)$. Then we let $(\mathbf{C m p l}(\Sigma) \downarrow \mathscr{G})$ denote the comma category of objects over $\mathscr{G}$. Thus $(\mathbf{C m p l}(\Sigma) \downarrow \mathscr{G})$ is the category whose objects are morphisms $\alpha$ of $\mathbf{C m p l}(\Sigma)$ whose codomain is $\mathscr{G}$ and whose morphisms from the object $\boldsymbol{\alpha}_{0}: \mathscr{F}_{0} \longrightarrow \mathscr{G}$ to the object $\boldsymbol{\alpha}_{1}: \mathscr{F}_{1} \longrightarrow \mathscr{G}$ are the ordered triples $\left(\boldsymbol{\alpha}_{0}, \boldsymbol{\beta}, \boldsymbol{\alpha}_{1}\right)$, which to shorten notation we identify with $\boldsymbol{\beta}$, where $\boldsymbol{\beta}$ is a morphism from $\mathscr{F}_{0}$ to $\mathscr{F}_{1}$ such that

$$
\alpha_{1} \diamond \beta=\alpha_{0},
$$




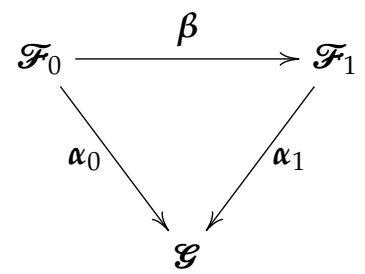

Figure 20. Morphism $\beta$ from $\alpha_{0}$ to $\alpha_{1}$ of the category $(\operatorname{Cmpl}(\Sigma) \downarrow \mathscr{G})$.

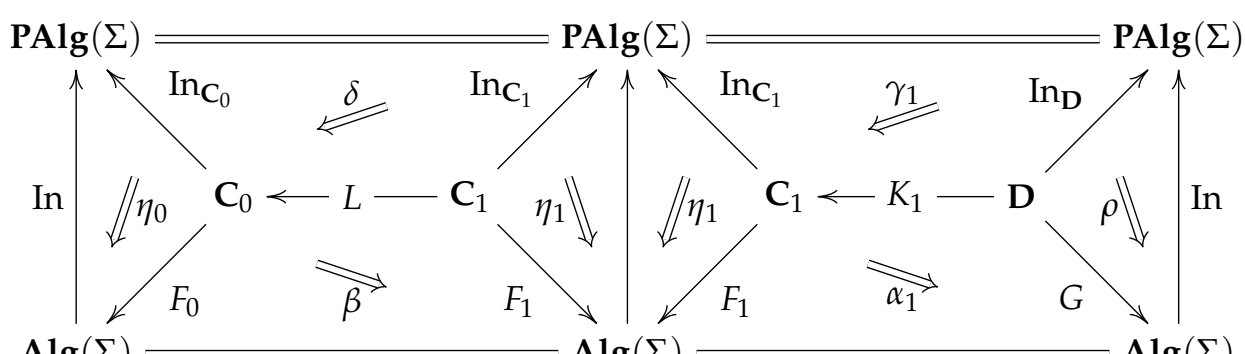

Figure 21. The composable morphisms of $\Sigma$-completions $\beta$ and $\alpha_{1}$.

i.e., such that the diagram in Figure 20 commutes.

Let us point out that, as a morphism of $(\operatorname{Cmpl}(\Sigma) \downarrow \mathscr{G})$, the domain of $\beta$ is $\boldsymbol{\alpha}_{0}$ and its codomain is $\boldsymbol{\alpha}_{1}$.

We will next show how to associate to a morphism $\beta$ of $(\operatorname{Cmpl}(\Sigma) \downarrow \mathscr{G})$ from an object $\alpha_{0}$ of $(\operatorname{Cmpl}(\Sigma) \downarrow \mathscr{G})$ to another object $\alpha_{1}$ of $(\operatorname{Cmpl}(\Sigma) \downarrow \mathscr{G})$ a natural transformation from $\mathrm{Y}_{\alpha_{0}}^{\mathscr{S}, 0}$, the Schmidt functor at $\mathbf{M o r}_{\mathrm{tw}}(\mathbf{D})$ associated to its domain, to $\mathrm{Y}_{\alpha_{1}}^{\mathscr{S}, 0}$, the Schmidt functor at $\mathbf{M o r}_{\mathrm{tw}}(\mathbf{D})$ associated to its codomain.

Proposition 45. Let $\mathscr{F}_{0}, \mathscr{F}_{1}$ and $\mathscr{G}$ be $\Sigma$-completions of $\mathrm{Cmpl}(\Sigma)$. Let $\boldsymbol{\alpha}_{0}$ be a morphism of $\operatorname{Cmpl}(\Sigma)$ from $\mathscr{F}_{0}$ to $\mathscr{G}, \alpha_{1}$ a morphism of $\operatorname{Cmpl}(\Sigma)$ form $\mathscr{F}_{1}$ to $\mathscr{G}$, and $\boldsymbol{\beta}$ a morphism of $(\operatorname{Cmpl}(\Sigma) \downarrow \mathscr{G})$ from $\boldsymbol{\alpha}_{0}$ to $\boldsymbol{\alpha}_{1}$, thus $\boldsymbol{\alpha}_{1} \diamond \boldsymbol{\beta}=\boldsymbol{\alpha}_{0}$. Then there exists a natural transformation from $\mathrm{Y}_{\alpha_{0}}^{\mathscr{G}, 0}$ to $\mathrm{Y}_{\alpha_{1}}^{\mathscr{G}, 0}$.

Proof. Let $\mathscr{F}_{0}, \mathscr{F}_{1}$ and $\mathscr{G}_{\text {stand for }}$

$$
\mathscr{F}_{0}=\left(\mathbf{C}_{0}, F_{0}, \eta_{0}\right), \mathscr{F}_{1}=\left(\mathbf{C}_{1}, F_{1}, \eta_{1}\right) \text { and } \mathscr{G}=(\mathbf{D}, G, \rho) \text {, respectively, }
$$

and let $\alpha_{0}, \alpha_{1}$ and $\beta$ stand for

$$
\boldsymbol{\alpha}_{0}=\left(K_{0}, \gamma_{0}, \alpha_{0}\right), \boldsymbol{\alpha}_{1}=\left(K_{1}, \gamma_{1}, \alpha_{1}\right) \text { and } \boldsymbol{\beta}=(L, \delta, \beta) \text {, respectively. }
$$

The reader is advised to consult the diagrams in Figures 21 and 22 for a better understanding of the entities under consideration.

On the other hand, from $\alpha_{1} \diamond \beta=\alpha_{0}$, we conclude that

$$
\begin{aligned}
& K_{0}=L \circ K_{1} ; \\
& \gamma_{0}=\left(\delta * \mathrm{id}_{K_{1}}\right) \circ \gamma_{1} ; \\
& \alpha_{0}=\alpha_{1} \circ\left(\beta * \mathrm{id}_{K_{1}}\right) .
\end{aligned}
$$

Moreover, by Proposition 44, the Schmidt endofunctors $Y_{\alpha_{0}, 0}^{\mathscr{E}, 0}$ and $Y_{\alpha_{1}, 0}^{\mathscr{E}, 0}$ relatives to $\boldsymbol{\alpha}_{0}$ 


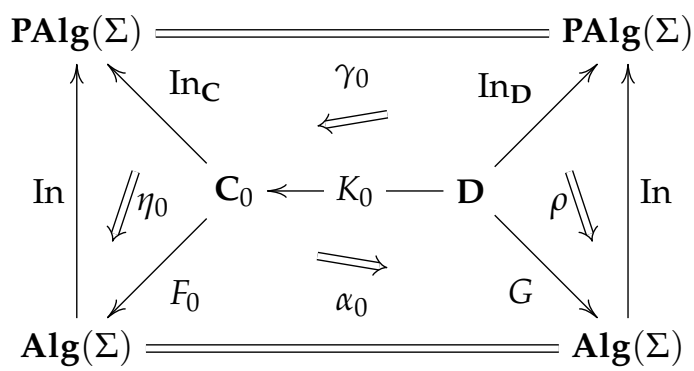

Figure 22. The morphism of $\Sigma$-completions $\alpha_{0}$.
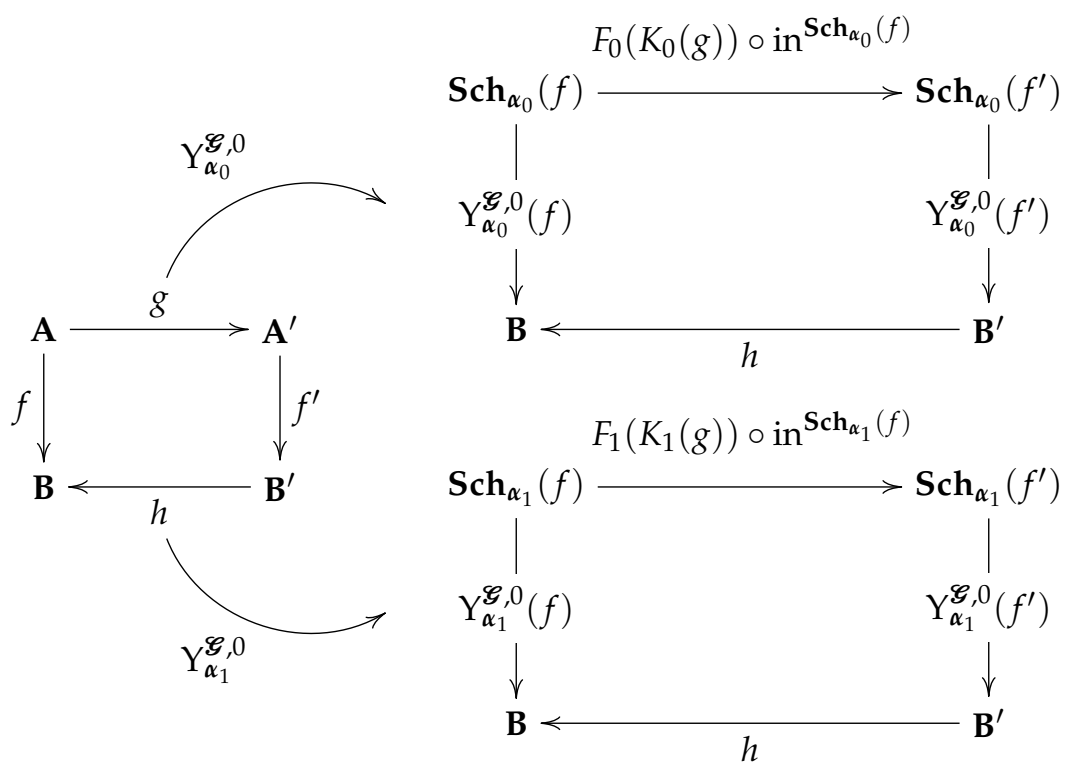

Figure 23. The functors $Y_{\alpha_{0}}^{\mathscr{G}, 0}$ and $Y_{\alpha_{1}}^{\mathscr{G}, 0}$.

and $\mathscr{G}$ and to $\alpha_{1}$ and $\mathscr{G}$, respectively, associate, to each object $f: \mathbf{A} \longrightarrow \mathbf{B}$ of $\mathbf{M o r}_{\mathrm{tw}}(\mathbf{D})$, the morphisms

$$
\mathrm{Y}_{\alpha_{0}}^{\mathscr{S}, 0}(f): \mathbf{S c h}_{\alpha_{0}}(f) \longrightarrow \mathbf{B} \text { and } Y_{\alpha_{1}}^{\mathscr{G}, 0}(f): \mathbf{S c h}_{\alpha_{1}}(f) \longrightarrow \mathbf{B} \text {, respectively, }
$$

and, to each morphism $(g, h)$ of $\mathbf{M o r}_{\text {tw }}(\mathbf{D})$ from the object $f: \mathbf{A} \longrightarrow \mathbf{B}$ to the object $f^{\prime}: \mathbf{A}^{\prime} \longrightarrow \mathbf{B}^{\prime}$, the morphisms

$$
\begin{aligned}
& \mathrm{Y}_{\boldsymbol{\alpha}_{0}}^{\mathscr{G}, 0}(g, h)=\left(F_{0}\left(K_{0}(g)\right) \circ \operatorname{in}^{\operatorname{Sch}_{\alpha_{0}}(f)}, h\right) \text { and } \\
& Y_{\boldsymbol{\alpha}_{1}}^{\mathscr{G}, 0}(g, h)=\left(F_{1}\left(K_{1}(g)\right) \circ \operatorname{in}^{\mathbf{S c h}_{\alpha_{1}}(f)}, h\right), \text { respectively. }
\end{aligned}
$$

The reader is advised to consult, in this respect, the diagrams in Figure 23.

After these preliminary remarks, we proceed, in what follows, to define a natural transformation $Y_{\beta}^{\mathscr{S}, 1}$ from the functor $Y_{\alpha_{0}}^{\mathscr{S}, 0}$ to the functor $Y_{\alpha_{1}}^{\mathscr{S}, 0}$, as shown in the diagram of Figure 24. To do it we should assign, in a natural way, to every object $f: \mathbf{A} \longrightarrow \mathbf{B}$ in $\mathbf{M o r}_{\mathrm{tw}}(\mathbf{D})$ a morphism $\mathbf{Y}_{\boldsymbol{\beta}, f}^{\mathscr{S}, 1}$ of $\mathbf{M o r}_{\mathrm{tw}}(\mathbf{D})$ from the homomorphism $\mathbf{Y}_{\boldsymbol{\alpha}_{0}, 0}^{\mathscr{E}}(f)$ to the homomorphism $\mathrm{Y}_{\alpha_{1}}^{\mathscr{G}, 0}(f)$, which will be the component of the natural transformation $\mathrm{Y}_{\beta}^{\mathscr{E}, 1}$ at $f$. Since, to define the morphisms of type $\mathrm{Y}_{\beta, f}^{\mathscr{G}, 1}$, we will make use both of the definition and the properties of the homomorphisms of type $\mathrm{Y}_{\alpha_{0}}^{\mathscr{S}, 0}$ and $\mathrm{Y}_{\alpha_{1}}^{\mathscr{S}, 0}$, the reader is advised to bear in mind Proposition 42. 


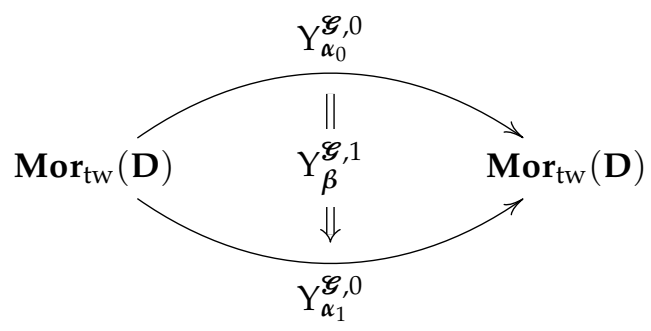

Figure 24. The natural transformation $Y_{\beta}^{\mathscr{S}, 1}$ from $Y_{\alpha_{0}}^{\mathscr{G}, 0}$ to $Y_{\alpha_{1}}^{\mathscr{G}, 0}$.

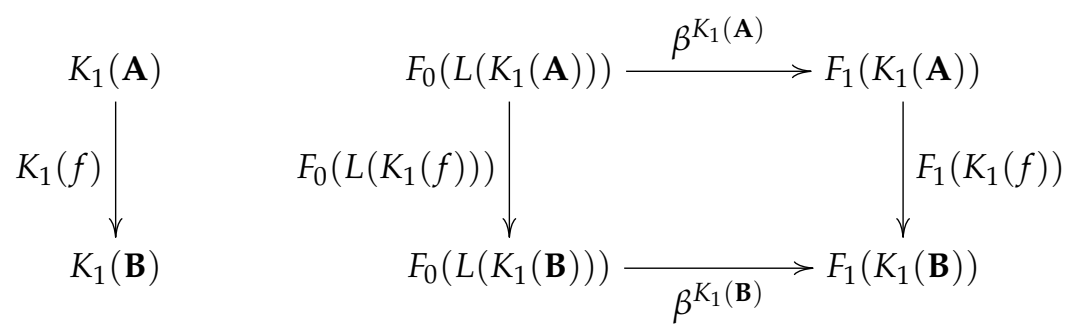

Figure 25. The natural transformation $\beta$ at $K_{1}(f)$.

To begin with, let us consider the value of the natural transformation $\beta$, from $F_{0} \circ L$ to $F_{1}$, at $K_{1}(\mathbf{A})$, i.e., the homomorphism $\beta^{K_{1}(\mathbf{A})}$ from $F_{0}\left(L\left(K_{1}(\mathbf{A})\right)\right)$ to $F_{1}\left(K_{1}(\mathbf{A})\right)$. This homomorphism is such that

$$
\beta^{K_{1}(\mathbf{B})} \circ F_{0}\left(L\left(K_{1}(f)\right)\right)=F_{1}\left(K_{1}(f)\right) \circ \beta^{K_{1}(\mathbf{A})},
$$

and we depict it in the diagram of Figure 25.

Let us recall that, by $\left(\dagger_{1}\right), K_{0}=L \circ K_{1}$. Therefore, $\beta^{K_{1}(\mathbf{A})}$ is a homomorphism from $F_{0}\left(K_{0}(\mathbf{A})\right)$ to $F_{1}\left(K_{1}(\mathbf{A})\right)$. Moreover, by Proposition $42, \mathbf{S c h}_{\boldsymbol{\alpha}_{0}}(f)$ is a relative subalgebra of $F_{0}\left(K_{0}(\mathbf{A})\right)$ and $\mathbf{S c h}_{\alpha_{1}}(f)$ is a relative subalgebra of $F_{1}\left(K_{1}(\mathbf{A})\right)$.

The following claim explains how the subalgebras $\mathbf{S c h}_{\boldsymbol{\alpha}_{0}}(f)$ and $\mathbf{S} \mathbf{c h} \boldsymbol{\alpha}_{\boldsymbol{\alpha}_{1}}(f)$ are related by means of $\beta^{K_{1}(\mathbf{A})}$.

Claim 5. The homomorphism $\beta^{K_{1}(\mathbf{A})}: F_{0}\left(K_{0}(\mathbf{A})\right) \longrightarrow F_{1}\left(K_{1}(\mathbf{A})\right)$ is such that $\beta^{K_{1}(\mathbf{A})}\left[\operatorname{Sch}_{\boldsymbol{\alpha}_{0}}(f)\right] \subseteq$ $\operatorname{Sch}_{\alpha_{1}}(f)$.

In fact, by Proposition 42, we have that

$$
\operatorname{Sch}_{\alpha_{0}}(f)=\left(G(f) \circ \alpha_{0}^{\mathbf{A}}\right)^{-1}\left[\rho^{\mathbf{B}}[B]\right] \text { and } \operatorname{Sch}_{\alpha_{1}}(f)=\left(G(f) \circ \alpha_{1}^{\mathbf{A}}\right)^{-1}\left[\rho^{\mathbf{B}}[B]\right] \text {. }
$$

Therefore, to prove that, for every $s \in S$ and every $z \in \operatorname{Sch}_{\alpha_{0}}(f)_{s} \beta_{s}^{K_{1}(\mathbf{A})}(z) \in \operatorname{Sch}_{\alpha_{1}}(f)_{s}$, it suffices to prove that

$$
G(f)_{s}\left(\alpha_{1, s}^{\mathbf{A}}\left(\beta_{s}^{K_{1}(\mathbf{A})}(z)\right)\right) \in \rho_{s}^{\mathbf{B}}\left[B_{S}\right]
$$

Let us note that the following chain of equalities holds

$$
\begin{aligned}
G(f) \circ \alpha_{1}^{\mathbf{A}} \circ \beta^{K_{1}(\mathbf{A})} & =G(f) \circ\left(\alpha_{1} \circ\left(\beta * \mathrm{id}_{K_{1}}\right)\right)^{\mathbf{A}} \\
& =G(f) \circ \alpha_{0}^{\mathbf{A}} .
\end{aligned}
$$

In the just stated chain of equalities, the first equality recovers the $\mathbf{A}$-th component of the natural transformation $\alpha_{1} \circ\left(\beta * \mathrm{id}_{K_{1}}\right)$; finally, the last equality follows according to $\left(\dagger_{3}\right)$. 


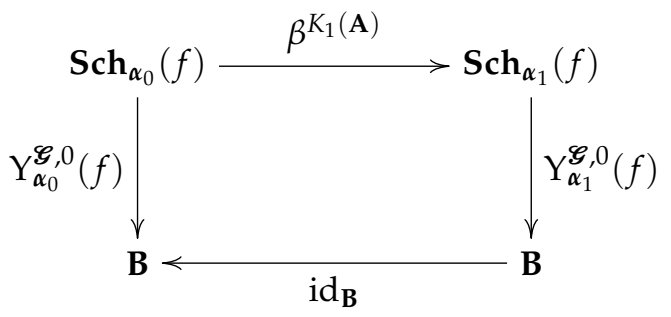

Figure 26. The morphism $Y_{\beta}^{\mathscr{E}, 1}$ at $f$.

Taking this into account and the definition of $\operatorname{Sch}_{\boldsymbol{\alpha}_{0}}(f)$, previously introduced, we conclude that $(\mathrm{Cl})$ holds.

This concludes the proof of Claim 5.

Notation 1. Let $f: \mathbf{A} \longrightarrow \mathbf{B}$ be any homomorphism of $\mathbf{D}$. Then, to shorten notation we let $\beta^{K_{1}(\mathbf{A})}$ stand for bires $\mathbf{S c h}_{\alpha_{0}}(f), \mathbf{S c h}_{\alpha_{1}}(f)\left(\beta^{K_{1}(\mathbf{A})}\right)$, the birestriction of $\beta^{K_{1}(\mathbf{A})}$ to $\mathbf{S c h}_{\boldsymbol{\alpha}_{0}}(f)$ and $\mathbf{S c h}_{\boldsymbol{\alpha}_{1}}(f)$.

Now, let $\mathrm{Y}_{\beta}^{\mathscr{S}, 1}$ be the mapping from $\operatorname{Mor}(\mathbf{D})$ to $\operatorname{Mor}(\mathbf{D})^{2}$, defined as follows: Let $f: \mathbf{A} \longrightarrow \mathbf{B}$ be a homomorphism, then $\mathrm{Y}_{\boldsymbol{\beta}, f}^{\mathscr{G}, 1}=\left(\beta^{K_{1}(\mathbf{A})}, \mathrm{id}_{\mathbf{B}}\right)$.

From here to the end of this proof, we proceed to show that $Y_{\beta}^{\mathscr{G}, 1}$, so defined, is, in fact, a natural transformation.

The following claim asserts that, for any homomorphism $f: \mathbf{A} \longrightarrow \mathbf{B}$ of $\mathbf{D}, \mathbf{Y}_{\boldsymbol{\beta}, f}^{\mathscr{E}, 1}$ is a morphism of $\mathbf{M o r}_{\mathrm{tw}}(\mathbf{D})$ from $\mathrm{Y}_{\boldsymbol{\alpha}_{0}}^{\mathscr{G}, 0}(f)$ to $\mathrm{Y}_{\boldsymbol{\alpha}_{1}}^{\mathscr{G}, 0}(f)$, i.e., that the diagram in Figure 26 commutes. From this it will follow that $\mathrm{Y}_{\beta}^{\mathscr{G}, 1}$ is a mapping from the set of objects of $\operatorname{Mor}_{\mathrm{tw}}(\mathbf{D})$ to the set of morphisms of $\operatorname{Mor}_{\mathrm{tw}}(\mathbf{D})$.

Claim 6. Let $f: \mathbf{A} \longrightarrow \mathbf{B}$ be a homomorphism of $\mathbf{D}$, then $\mathbf{Y}_{\boldsymbol{\beta}, f}^{\mathscr{G}, 1}$ is a morphism of $\mathbf{M o r}_{\mathrm{tw}}(\mathbf{D})$ from $\mathrm{Y}_{\boldsymbol{\alpha}_{0}}^{\mathscr{G}, 0}(f)$ to $\mathrm{Y}_{\boldsymbol{\alpha}_{1}}^{\mathscr{G}, 0}(f)$.

In the proof of this Claim we take into account Claim 5. (The reader is advised to consult the diagram in Figure 26 for a better understanding of the elements under consideration.)

We need to prove that

$$
\mathrm{Y}_{\alpha_{0}}^{\mathscr{G}, 0}(f)=\mathrm{id}_{\mathbf{B}} \circ \mathrm{Y}_{\boldsymbol{\alpha}_{1}}^{\mathscr{G}, 0}(f) \circ \beta^{K_{1}(\mathbf{A})}
$$

But, by Proposition 42, the many-sorted partial $\Sigma$-algebra $\mathbf{S c h}_{\boldsymbol{\alpha}_{0}}(f)$ is $\eta_{0}^{K_{0}(\mathbf{A})}\left[\gamma_{0}^{\mathbf{A}}[A]\right]$ generated and $\operatorname{id}_{\mathbf{B}}, Y_{\boldsymbol{\alpha}_{1}}^{\mathscr{G}, 0}(f), \beta^{K_{1}(\mathbf{A})}$ and $\mathrm{Y}_{\boldsymbol{\alpha}_{0}}^{\mathscr{G}, 0}(f)$ are homomorphisms. Therefore, it suffices to prove that the following equality holds

$$
\mathrm{Y}_{\boldsymbol{\alpha}_{0}}^{\mathscr{G}, 0}(f) \circ \eta_{0}^{K_{0}(\mathbf{A})} \circ \gamma_{0}^{\mathbf{A}}=\mathrm{id}_{\mathbf{B}} \circ \mathrm{Y}_{\boldsymbol{\alpha}_{1}}^{\mathscr{G}, 0}(f) \circ \beta^{K_{1}(\mathbf{A})} \circ \eta_{0}^{K_{0}(\mathbf{A})} \circ \gamma_{0}^{\mathbf{A}} .
$$

Starting from the right hand side of the above equation, we have that the following chain of equalities holds 


$$
\begin{aligned}
& \operatorname{id}_{\mathbf{B}} \circ Y_{\alpha_{1}}^{\mathscr{E}, 0}(f) \circ \beta^{K_{1}(\mathbf{A})} \circ \eta_{0}^{K_{0}(\mathbf{A})} \circ \gamma_{0}^{\mathbf{A}} \\
& =\mathrm{Y}_{\alpha_{1}}^{\mathscr{S}, 0}(f) \circ \beta^{K_{1}(\mathbf{A})} \circ \eta_{0}^{K_{0}(\mathbf{A})} \circ \gamma_{0}^{\mathbf{A}} \\
& =\mathrm{Y}_{\boldsymbol{\alpha}_{1}}^{\mathscr{G}, 0}(f) \circ \beta^{K_{1}(\mathbf{A})} \circ \eta_{0}^{K_{0}(\mathbf{A})} \circ\left(\left(\delta * \mathrm{id}_{K_{1}}\right) \circ \gamma_{1}\right)^{\mathbf{A}} \\
& =Y_{\alpha_{1}}^{\mathscr{S}, 0}(f) \circ \beta^{K_{1}(\mathbf{A})} \circ \eta_{0}^{K_{0}(\mathbf{A})} \circ \delta^{K_{1}(\mathbf{A})} \circ \gamma_{1}^{\mathbf{A}} \\
& =\mathrm{Y}_{\boldsymbol{\alpha}_{1}}^{\mathscr{G}, 0}(f) \circ \beta^{K_{1}(\mathbf{A})} \circ \eta_{0}^{L\left(K_{1}(\mathbf{A})\right)} \circ \delta^{K_{1}(\mathbf{A})} \circ \gamma_{1}^{\mathbf{A}} \\
& =\mathrm{Y}_{\alpha_{1}}^{\mathscr{S}, 0}(f) \circ \eta_{1}^{K_{1}(\mathbf{A})} \circ \gamma_{1}^{\mathbf{A}} \\
& =f \\
& =\mathrm{Y}_{\boldsymbol{\alpha}_{0}}^{\mathscr{E}, 0}(f) \circ \eta_{0}^{K_{0}(\mathbf{A})} \circ \gamma_{0}^{\mathbf{A}} \text {. }
\end{aligned}
$$

In the just stated chain of equalities, the first equality applies the identity mapping at B; the second equality follows from $\left(t_{2}\right)$; the third equality recovers the $\mathbf{A}$-th component of the natural transformation $\left(\delta * \mathrm{id}_{K_{1}}\right) \circ \gamma_{1}$; the fourth equality follows from $\left(t_{1}\right)$; the fifth equality follows from the fact that $\beta$ is a morphism from the $\Sigma$-completion $\mathscr{F}_{0}$ to the $\Sigma$-completion $\mathscr{F}_{1}$; the sixth equality follows from Proposition 42 at $\mathrm{Y}_{\alpha_{1}}^{\mathscr{G}, 0}(f)$; finally, the last equality follows from Proposition 42 at $\mathrm{Y}_{\boldsymbol{\alpha}_{0}, 0}^{\mathscr{S}, 0}(f)$.

This completes the proof of Claim 6.

We can now prove that $\mathrm{Y}_{\beta}^{\mathscr{S}, 1}$ is the desired natural transformation.

Claim 7. $\mathrm{Y}_{\boldsymbol{\beta}}^{\mathscr{S}, 1}$ is a natural transformation from $\mathrm{Y}_{\boldsymbol{\alpha}_{0}}^{\mathscr{S}, 0}$ to $\mathrm{Y}_{\boldsymbol{\alpha}_{1}}^{\mathscr{G}, 0}$.

To prove Claim 7, we need to show that, for every pair of homomorphisms $f: \mathbf{A} \longrightarrow \mathbf{B}$ and $f^{\prime}: \mathbf{A}^{\prime} \longrightarrow \mathbf{B}^{\prime}$ of $\mathbf{D}$ and every morphism $(g, h)$ from $f$ to $f^{\prime}$ of $\mathbf{M o r}_{\mathrm{tw}}(\mathbf{D})$, the following equality holds

$$
\mathrm{Y}_{\boldsymbol{\beta}, f^{\prime}}^{\mathscr{G}, 1} \circ \mathrm{Y}_{\boldsymbol{\alpha}_{0}}^{\mathscr{S}, 0}(g, h)=\mathrm{Y}_{\boldsymbol{\alpha}_{1}}^{\mathscr{G}, 0}(g, h) \circ \mathrm{Y}_{\boldsymbol{\beta}, f}^{\mathscr{G}, 1}
$$

But, taking into account the definitions of the four components of the above equality, to show that it is fulfilled is equivalent to showing that the following equalities are satisfied

$$
\begin{aligned}
& F_{1}\left(K_{1}(g)\right) \circ \operatorname{in}^{\mathbf{S c h}_{\alpha_{1}}(f)} \circ \beta^{K_{1}(\mathbf{A})}=\beta^{K_{1}\left(\mathbf{A}^{\prime}\right)} \circ F_{0}\left(K_{0}(g)\right) \circ \operatorname{in}^{\mathbf{S c h}_{\alpha_{0}}(f)} ; \\
& \operatorname{id}_{\mathbf{B}} \circ h=h \circ \mathrm{id}_{\mathbf{B}^{\prime}} \text {. }
\end{aligned}
$$

The reader is advised to consult the diagram in Figure 27 for a better understanding of the elements under consideration.

The equation $\mathrm{id}_{\mathbf{B}} \circ h=h \circ \mathrm{id}_{\mathbf{B}^{\prime}}$ trivially holds. Therefore, it suffices to prove the first equation. In this regard, since, by Proposition 42, the partial $\Sigma$-algebra $\mathbf{S c h}_{\boldsymbol{\alpha}_{0}}(f)$ is $\eta_{0}^{K_{0}(\mathbf{A})}\left[\gamma_{0}^{\mathbf{A}}[A]\right]$-generated and since $F_{1}\left(K_{1}(g)\right)$, in $\mathbf{S c h}_{\alpha_{1}}(f), \beta^{K_{1}(\mathbf{A})}, \beta^{K_{1}\left(\mathbf{A}^{\prime}\right)}, F_{0}\left(K_{0}(g)\right)$, and in $\operatorname{Sch}_{\alpha_{0}}(f)$ are homomorphisms, it suffices to prove that

$$
\begin{aligned}
F_{1}\left(K_{1}(g)\right) \circ \operatorname{in}^{\mathbf{S c h}_{\alpha_{1}}(f)} \circ \beta^{K_{1}(\mathbf{A})} \circ \eta_{0}^{K_{0}(\mathbf{A})} \circ \gamma_{0}^{\mathbf{A}} & \\
& =\beta^{K_{1}\left(\mathbf{A}^{\prime}\right)} \circ F_{0}\left(K_{0}(g)\right) \circ \operatorname{in}^{\mathbf{S c h}_{\alpha_{0}}(f)} \circ \eta_{0}^{K_{0}(\mathbf{A})} \circ \gamma_{0}^{\mathbf{A} .}
\end{aligned}
$$

Starting from the left hand side of the above equation, we have that the following chain of equalities holds

$$
\begin{aligned}
& F_{1}\left(K_{1}(g)\right) \circ \operatorname{in}^{\mathbf{S c h}_{\alpha_{1}}(f)} \circ \beta^{K_{1}(\mathbf{A})} \circ \eta_{0}^{K_{0}(\mathbf{A})} \circ \gamma_{0}^{\mathbf{A}} \\
& \quad=F_{1}\left(K_{1}(g)\right) \circ \operatorname{in}^{\mathbf{S c h}_{\alpha_{1}}(f)} \circ \beta^{K_{1}(\mathbf{A})} \circ \eta_{0}^{K_{0}(\mathbf{A})} \circ\left(\left(\delta * \mathrm{id}_{K_{1}}\right) \circ \gamma_{1}\right)^{\mathbf{A}}
\end{aligned}
$$




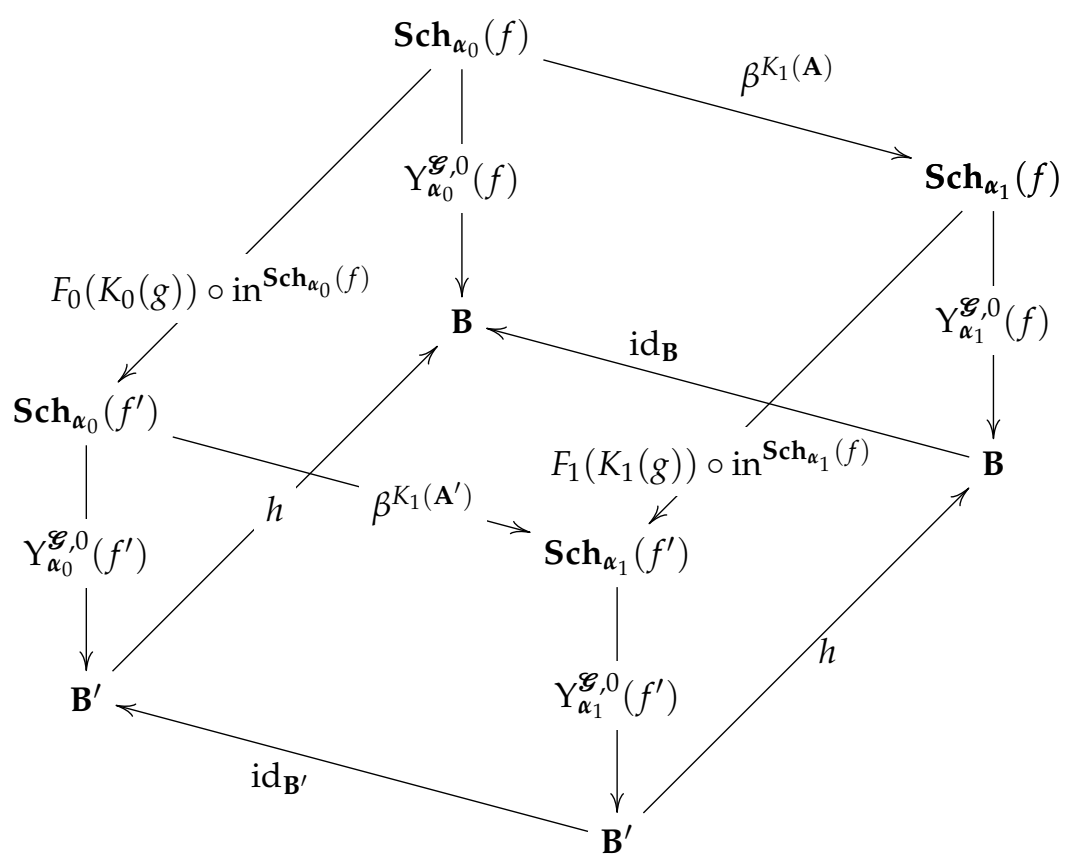

Figure 27. The natural transformation $Y_{\beta}^{\mathscr{S}, 1}$ at a morphism $(g, h)$ from $f$ to $f^{\prime}$ of $\operatorname{Mor}_{\mathrm{tw}}(\mathbf{D})$.

$$
\begin{aligned}
& =F_{1}\left(K_{1}(g)\right) \circ \operatorname{in}^{\mathbf{S c h}_{\alpha_{1}}(f)} \circ \beta^{K_{1}(\mathbf{A})} \circ \eta_{0}^{K_{0}(\mathbf{A})} \circ \delta^{K_{1}(\mathbf{A})} \circ \gamma_{1}^{\mathbf{A}} \\
& =F_{1}\left(K_{1}(g)\right) \circ \operatorname{in}^{\mathbf{S c h}_{\alpha_{1}}(f)} \circ \beta^{K_{1}(\mathbf{A})} \circ \eta_{0}^{L\left(K_{1}(\mathbf{A})\right)} \circ \delta^{K_{1}(\mathbf{A})} \circ \gamma_{1}^{\mathbf{A}} \\
& =F_{1}\left(K_{1}(g)\right) \circ \operatorname{in}^{\mathbf{S c h}_{\alpha_{1}}(f)} \circ \eta_{1}^{K_{1}(\mathbf{A})} \circ \gamma_{1}^{\mathbf{A}} \\
& =F_{1}\left(K_{1}(g)\right) \circ \eta_{1}^{K_{1}(\mathbf{A})} \circ \gamma_{1}^{\mathbf{A}} \\
& =\eta_{1}^{K_{1}\left(\mathbf{A}^{\prime}\right)} \circ K_{1}(g) \circ \gamma_{1}^{\mathbf{A}} \\
& =\beta^{K_{1}\left(\mathbf{A}^{\prime}\right)} \circ \eta_{0}^{L\left(K_{1}\left(\mathbf{A}^{\prime}\right)\right)} \circ \delta^{K_{1}\left(\mathbf{A}^{\prime}\right)} \circ K_{1}(g) \circ \gamma_{1}^{\mathbf{A}} \\
& =\beta^{K_{1}\left(\mathbf{A}^{\prime}\right)} \circ \eta_{0}^{L\left(K_{1}\left(\mathbf{A}^{\prime}\right)\right)} \circ L\left(K_{1}(g)\right) \circ \delta^{K_{1}(\mathbf{A})} \circ \gamma_{1}^{\mathbf{A}} \\
& =\beta^{K_{1}\left(\mathbf{A}^{\prime}\right)} \circ F_{0}\left(L\left(K_{1}(g)\right)\right) \circ \eta_{0}^{L\left(K_{1}(\mathbf{A})\right)} \circ \delta^{K_{1}(\mathbf{A})} \circ \gamma_{1}^{\mathbf{A}} \\
& =\beta^{K_{1}\left(\mathbf{A}^{\prime}\right)} \circ F_{0}\left(K_{0}(g)\right) \circ \eta_{0}^{K_{0}(\mathbf{A})} \circ \delta^{K_{1}(\mathbf{A})} \circ \gamma_{1}^{\mathbf{A}} \\
& =\beta^{K_{1}\left(\mathbf{A}^{\prime}\right)} \circ F_{0}\left(K_{0}(g)\right) \circ \eta_{0}^{K_{0}(\mathbf{A})} \circ\left(\left(\delta * \mathrm{id}_{K_{1}}\right) \circ \gamma_{1}\right)^{\mathbf{A}} \\
& =\beta^{K_{1}\left(\mathbf{A}^{\prime}\right)} \circ F_{0}\left(K_{0}(g)\right) \circ \eta_{0}^{K_{0}(\mathbf{A})} \circ \gamma_{0}^{\mathbf{A}} \\
& =\beta^{K_{1}\left(\mathbf{A}^{\prime}\right)} \circ F_{0}\left(K_{0}(g)\right) \circ \operatorname{in}^{\mathbf{S c h}_{\alpha_{0}}(f)} \circ \eta_{0}^{K_{0}(\mathbf{A})} \circ \gamma_{0}^{\mathbf{A}} .
\end{aligned}
$$

In the just stated chain of equalities, the first equality follows from $\left(t_{2}\right)$; the second equality recovers the $\mathbf{A}$-th component at the natural transformation $\left(\delta * \mathrm{id}_{K_{1}}\right) \circ \gamma_{1}$; the third equality follows from $\left(t_{1}\right)$; the fourth equality follows from the fact that $\beta$ is a morphism from the $\Sigma$-completion $\mathscr{F}_{0}$ to the $\Sigma$-completion $\mathscr{F}_{1}$; the fifth equality follows from Proposition 42; the sixth equality follows from the fact that $\eta_{1}$ is a natural transformation from $\operatorname{In}_{\mathrm{C}_{1}}$ to $\operatorname{In}_{\operatorname{Alg}(\Sigma)} \circ F_{1}$; the seventh equality follows from the fact that $\beta$ is a morphism from the $\Sigma$-completion $\mathscr{F}_{0}$ to the $\Sigma$-completion $\mathscr{F}_{1}$; the eighth equality follows from the fact that $\delta$ is a natural transformation from $\operatorname{In}_{\mathrm{C}_{1}}$ to $\operatorname{In}_{\mathrm{C}_{0}} \circ L$; the ninth equality follows from the fact that $\eta_{0}$ is a natural transformation from $\operatorname{In}_{\mathbf{C}_{0}}$ to $\operatorname{In}_{\mathbf{A l g}(\Sigma)} \circ F_{0}$; the tenth equality follows from $\left(t_{1}\right)$; the eleventh equality recovers the A-th component at the natural transformation $\left(\delta * \mathrm{id}_{K_{1}}\right) \circ \gamma_{1}$; the twelfth equality follows from $\left(\dagger_{2}\right)$; and finally, the last equality follows from Proposition 42.

This concludes the proof of Claim 7. 

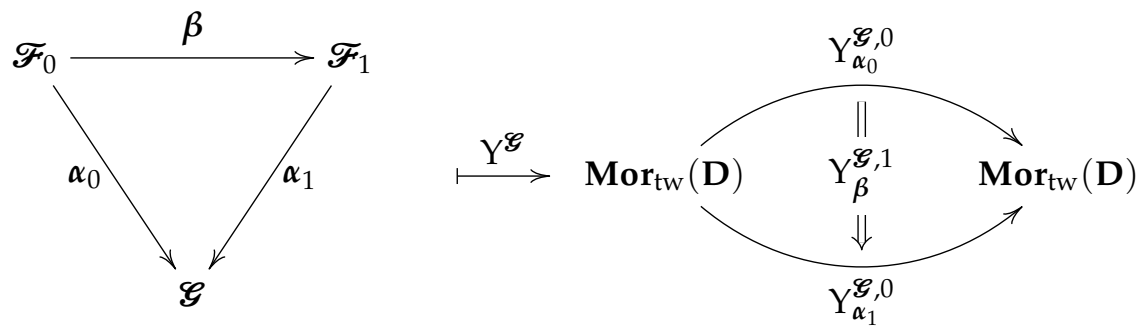

Figure 28. The functor $Y^{\mathscr{G}}$.

This completes the proof of Proposition 45.

The result established in Proposition 45 leads us to consider it reasonable that, for every $\Sigma$-completion $\mathscr{G}=(\mathbf{D}, G, \rho)$ of $\mathbf{C m p l}(\Sigma)$, there exists a functor from $(\mathbf{C m p l}(\Sigma) \downarrow \mathscr{G})$ to $\operatorname{End}\left(\operatorname{Mor}_{\mathrm{tw}}(\mathbf{D})\right)$. The remainder of this section will be devoted to prove this statement.

Definition 45. Let $\mathbf{C}$ be a category. We denote by $\operatorname{End}(\mathbf{C})$ the category whose objects are the endofunctors of $\mathbf{C}$ and whose morphisms are the natural transformations between these endofunctors (the vertical composition of natural transformations is indeed associative, and there is an identity natural transformation from every endofunctor to itself).

Definition 46. Let $\mathscr{G}=(\mathbf{D}, G, \rho)$ be a $\sum$-completion. Let $\mathrm{Y}^{\mathscr{G}}$ denote the ordered pair $\left(\mathrm{Y}^{\mathscr{G}, 0}, \mathrm{Y}^{\mathscr{G}, 1}\right)$ where

1. $\mathrm{Y}^{\mathscr{G}, 0}$ is the mapping that sends an object $\boldsymbol{\alpha}: \mathscr{F} \longrightarrow \mathscr{G}$ of $(\mathbf{C m p l}(\Sigma) \downarrow \mathscr{G})$, i.e., a morphism of $\Sigma$-completion of $\mathbf{C m p l}(\Sigma)$ with codomain $\mathscr{G}$, to the endofunctor $\mathrm{Y}_{\alpha}^{\mathscr{S}, 0}$ of $\operatorname{End}\left(\operatorname{Mor}_{\mathrm{tw}}(\mathbf{D})\right)$, and

2. $\mathrm{Y}^{\mathscr{G}, 1}$ is the mapping that sends a morphism $\boldsymbol{\beta}$ of $(\mathbf{C m p l}(\Sigma) \downarrow \mathscr{G})$ from the object $\boldsymbol{\alpha}_{0}: \mathscr{F}_{0} \longrightarrow \mathscr{G}$ to the object $\alpha_{1}: \mathscr{F}_{1} \longrightarrow \mathscr{G}$, i.e., a morphism of $\Sigma$-completions $\beta: \mathscr{F}_{0} \longrightarrow \mathscr{F}_{1}$ satisfying the equation $\boldsymbol{\alpha}_{1} \diamond \boldsymbol{\beta}=\boldsymbol{\alpha}_{0}$, to the natural transformation $\mathrm{Y}_{\boldsymbol{\beta}}^{\mathscr{E}, 1}$ from $\mathrm{Y}_{\boldsymbol{\alpha}_{0}}^{\mathscr{S}, 0}$ to $\mathrm{Y}_{\boldsymbol{\alpha}_{1}}^{\mathscr{E}, 0}$ defined in Proposition 45.

The transformation associated to the morphism $\boldsymbol{\beta}$ (from the object $\boldsymbol{\alpha}_{0}$ to the object $\boldsymbol{\alpha}_{1}$ ) of $(\mathbf{C m p l}(\Sigma) \downarrow \mathscr{G})$ is represented in the diagram of Figure 28.

In the following proposition we prove that $\mathrm{Y}^{\mathscr{G}}$ is, indeed, a functor.

Proposition 46. Let $\mathscr{G}=(\mathbf{D}, G, \rho)$ be a $\sum$-completion. Then $\mathrm{Y}^{\mathscr{G}}$, as introduced in Definition 46, is a functor from $(\mathbf{C m p l}(\Sigma) \downarrow \mathscr{G})$ to $\operatorname{End}\left(\operatorname{Mor}_{\mathrm{tw}}(\mathbf{D})\right)$.

Proof. We have divided the proof into a series of claims.

Claim 8. $\mathrm{Y}^{\mathscr{G}}$ preserves identities.

Let $\alpha=(K, \gamma, \alpha)$ be a morphism of $\Sigma$-completions from $\mathscr{F}=(\mathbf{C}, F, \eta)$ to $\mathscr{G}$. We need to prove that the following equality holds

$$
\mathrm{Y}_{\mathrm{id}_{\alpha}}^{\mathscr{G}, 1}=\mathrm{id}_{\mathrm{Y}_{\alpha}, 0}
$$

But, $\operatorname{id}_{\alpha}$, the identity morphism at $\alpha$ in $(\operatorname{Cmpl}(\Sigma) \downarrow \mathscr{G})$, is

$$
\mathscr{F}_{\mathscr{F}}=\left(\operatorname{Id}_{\mathrm{C}}, \mathrm{id}_{\mathrm{In}_{\mathrm{C}}}, \mathrm{id}_{F}\right)
$$

the identity morphism at $\mathscr{F}$ (recall the convetion stated in Definition 44). 
Moreover, for a homomorphism $f: \mathbf{A} \longrightarrow \mathbf{B}$ of $\mathbf{D}$, we have that

$$
\mathrm{Y}_{\mathrm{id}_{\alpha, f}}^{\mathscr{G}, 1}=\left(\mathrm{id}_{F}^{K(\mathbf{A})}, \mathrm{id}_{\mathbf{B}}\right),
$$

where, by Proposition 45, $\mathrm{id}_{F}^{K(\mathbf{A})}$ stands for the birestriction of $\mathrm{id}_{F}^{K(\mathbf{A})}$ to $\mathbf{S c h}_{\boldsymbol{\alpha}}(f)$. Therefore,

$$
\mathrm{Y}_{\mathrm{id}_{\alpha}, f}^{\mathscr{G}, 1}=\left(\operatorname{id}_{\mathbf{S c h}_{\alpha}(f)}, \mathrm{id}_{\mathbf{B}}\right)=\mathrm{id}_{\mathrm{Y}_{\alpha}^{\mathscr{g}, 0}}^{f} .
$$

This completes the proof of Claim 8.

Claim 9. $\mathrm{Y}^{\mathscr{G}}$ preserves compositions.

Let $\alpha_{0}, \alpha_{1}$ and $\alpha_{2}$ be objects of $(\operatorname{Cmpl}(\Sigma) \downarrow \mathscr{G})$ of the form

$$
\alpha_{0}: \mathscr{F}_{0} \longrightarrow \mathscr{G}, \alpha_{1}: \mathscr{F}_{1} \longrightarrow \mathscr{G} \text { and } \alpha_{2}: \mathscr{F}_{2} \longrightarrow \mathscr{G},
$$

where $\alpha_{0}, \alpha_{1}$ and $\alpha_{2}$ stand for

$$
\boldsymbol{\alpha}_{0}=\left(K_{0}, \gamma_{0}, \alpha_{0}\right), \boldsymbol{\alpha}_{1}=\left(K_{1}, \gamma_{1}, \alpha_{1}\right) \text { and } \alpha_{2}=\left(K_{2}, \gamma_{2}, \alpha_{2}\right), \text { respectively, }
$$

and let $\beta_{0}$ and $\beta_{1}$ be morphisms in $(\operatorname{Cmpl}(\Sigma) \downarrow \mathscr{G})$ of the form

$$
\beta_{0}: \mathscr{F}_{0} \longrightarrow \mathscr{F}_{1} \text { and } \beta_{1}: \mathscr{F}_{1} \longrightarrow \mathscr{F}_{2}
$$

where $\beta_{0}$ and $\beta_{1}$ stand for

$$
\boldsymbol{\beta}_{0}=\left(L_{0}, \delta_{0}, \beta_{0}\right) \text { and } \beta_{1}=\left(L_{1}, \delta_{1}, \beta_{1}\right) \text {, respectively. }
$$

We need to prove that the following equality holds

$$
\mathrm{Y}_{\beta_{1} \diamond \beta_{0}}^{\mathscr{S}, 1}=\mathrm{Y}_{\beta_{1}}^{\mathscr{S}, 1} \circ \mathrm{Y}_{\beta_{0}}^{\mathscr{S}, 1}
$$

But the composition of two morphisms in $(\operatorname{Cmpl}(\Sigma) \downarrow \mathscr{G})$ is given by the composition of the morphisms in $\operatorname{Cmpl}(\Sigma)$ which, by Definition 40, is

$$
\boldsymbol{\beta}_{1} \diamond \boldsymbol{\beta}_{0}=\left(L_{1}, \delta_{1}, \beta_{1}\right) \diamond\left(L_{0}, \delta_{0}, \beta_{0}\right)=\left(L_{0} \circ L_{1},\left(\delta_{0} * \mathrm{id}_{L_{1}}\right) \circ \delta_{1}, \beta_{1} \circ\left(\beta_{0} * \mathrm{id}_{L_{1}}\right)\right) \text {. }
$$

Thus, for a morphism $f: \mathbf{A} \longrightarrow \mathbf{B}$ of $\mathbf{D}$, we have that

$$
\mathrm{Y}_{\boldsymbol{\beta}_{1} \diamond \boldsymbol{\beta}_{0}, f}^{\mathscr{S}, 1}=\left(\left(\beta_{1} \circ\left(\beta_{0} * \mathrm{id}_{L_{1}}\right)\right)^{K_{2}(\mathbf{A})}, \mathrm{id}_{\mathbf{B}}\right)=\left(\beta_{1}^{K_{2}(\mathbf{A})} \circ \beta_{0}^{L_{1}\left(K_{2}\left(\mathbf{A}^{\prime}\right)\right)}, \mathrm{id}_{\mathbf{B}}\right) .
$$

Moreover, we have that

$$
\mathrm{Y}_{\beta_{1}, f}^{\mathscr{G}, 1}=\left(\beta_{1}^{K_{2}(\mathbf{A})}, \mathrm{id}_{\mathbf{B}}\right) \text { and } \mathrm{Y}_{\beta_{0}, f}^{\mathscr{G}, 1}=\left(\beta_{0}^{K_{1}(\mathbf{A})}, \mathrm{id}_{\mathbf{B}}\right) .
$$

But, since $\beta_{1}$ is a morphism of $(\operatorname{Cmpl}(\Sigma) \downarrow \mathscr{G})$ from $\alpha_{1}$ to $\alpha_{2}$, we have, in particular, that $K_{1}=L_{1} \circ K_{2}$.

Thus, the following chain of equalities holds

$$
\mathrm{Y}_{\boldsymbol{\beta}_{1}, f}^{\mathscr{G}, 1} \circ \mathrm{Y}_{\boldsymbol{\beta}_{0}, f}^{\mathscr{G}, 1}=\left(\beta_{1}^{K_{2}(\mathbf{A})}, \mathrm{id}_{\mathbf{B}}\right) \circ\left(\beta_{0}^{K_{1}(\mathbf{A})}, \mathrm{id}_{\mathbf{B}}\right)=\left(\beta_{1}^{K_{2}(\mathbf{A})} \circ \beta_{0}^{L_{1}\left(K_{2}\left(\mathbf{A}^{\prime}\right)\right)}, \mathrm{id}_{\mathbf{B}}\right) .
$$

This completes the proof of Claim 9.

This concludes the proof of Proposition 46.

In view of the above, it seems natural to ask the following question: Is the family of functors $\left(\mathrm{Y}^{\mathscr{G}}\right)_{\mathscr{G} \in \mathrm{Ob}(\mathrm{Cmpl}(\Sigma))}$ the object mapping of a functor from $\mathbf{C m p l}(\Sigma)$ ? We think that 
this is not the case and that we are facing a situation similar to the one that occurs in group theory, in which, e.g., the centre of a group does not determine a subfunctor of the identity functor at the category of groups (it is true that if one chooses to restrict Grp, the category of groups, by using as morphisms the surjective homomorphisms, one obtains a functor, but, for the case at hand, such a solution does not seem likely to work). In this case, the most we can say is that the objects $\mathscr{G}$ of the category $\operatorname{Cmpl}(\Sigma)$ play the role of parameters for the functors $\mathrm{Y}^{\mathscr{H}}$, while the morphisms of $\mathrm{Cmpl}(\Sigma)$ are used to act as morphisms of $(\operatorname{Cmpl}(\Sigma) \downarrow \mathscr{G})$, the comma categories which are the domains of such functors.

Author Contributions: Both authors equally contributed in every aspect of the writing of this article. Both authors have read and agreed to the published version of the manuscript.

Funding: The second author was supported by the Ministerio de Ciencia, Innovación y Universidades and the Agencia Estatal de Investigación, Spain, and FEDER, European Union, under Grant PGC2018095140-B-I00.

Conflicts of Interest: The authors declare no conflict of interest.

\section{References}

1. G. M. Bergman, An invitation to general algebra and universal constructions. Second edition. Universitext. Springer, Cham, 2015.

2. N. Bourbaki, Théorie des ensembles. Hermann, Paris, 1970.

3. P. Burmeister and J. Schmidt, On the completion of partial algebras. Colloq. Math. 17 (1967), pp. $235-245$.

4. P. Burmeister, Free partial algebras. J. Reine Angew. Math. 241 (1970), pp. 75-86.

5. P. Burmeister, Partial algebras-survey of a unifying approach towards a twovalued model theory for partial algebras. Algebra Universalis, 15 (1982), pp. 306-358.

6. P. Burmeister, A model theoretic oriented approach to partial algebras. Introduction to theory and application of partial algebras. Part I. Mathematical Research, 32. Akademie-Verlag, Berlin, 1986.

7. P. Burmeister, Lecture Notes on Universal Algebra-Many-Sorted Partial Algebras. Fragment of lectures hold in the summer terms of 1994, 1996, 1998, 2000 and 2002 at the Darmstadt University of Technology. Download via http://www.mathematik.tudarmstadt.de/ burmeister/

8. S. Burris and H. P. Sankappanavar, A course in universal algebra. Springer-Verlag, New York-Berlin, 1981.

9. S. Eilenberg and S. MacLane, General theory of natural equivalences. Trans. Amer. Math. Soc. 58 (1945), pp. $231-294$.

10. J. Goguen, J. Thatcher, and E. Wagner, An initial algebra approach to the specification, correctness, and implementation of abastract data types. In R. Yeh, editor. Current Trends in Programming Methodology, IV, Chapter 5, pp. 80-149, Prentice Hall, New Jersey, 1978.

11. G. Grätzer, Universal algebra. With appendices by Grätzer, Bjarni Jónsson, Walter Taylor, Robert W. Quackenbush, Günter H. Wenzel, and Grätzer and W. A. Lampe. Revised reprint of the 1979 second edition. Springer, New York, 2008.

12. H. Herrlich and G. E. Strecker, Category theory: an introduction. Allyn and Bacon Series in Advanced Mathematics. Allyn and Bacon Inc., Boston, Mass., 1973.

13. A. G. Kurosh, Algèbre générale. (French) Traduit du Russe par J.-P. Peaudecerf avec la participation de G. Lallement Collection Universitaire de Mathématiques, No. 22. Dunod, Paris, 1967.

14. S. Mac Lane, Categories for the working mathematician. 2nd ed. Springer-Verlag, New York, 1998.

15. G. Matthiessen, Theorie der Heterogenen Algebren, Mathematik-Arbeitspapiere, Nr. 3, Universität Bremen Teil A, Mathematische Forchungspapiere, 1976.

16. J. Schmidt, A general existence theorem on partial algebras and its special cases. Colloq. Math. 14 (1966) pp. $73-87$.

17. J. Schmidt, A homomorphism theorem for partial algebras. Colloq. Math. 21 (1970) pp. 5-21.

18. W. Wechler, Universal algebra for computer scientists. Springer-Verlag, Berlin, 1992. 